Portland State University

PDXScholar

$1-1-1985$

\title{
Spatial clustering of sector linked industry in an urban economy
}

Colleen Greer Acres

Portland State University

Follow this and additional works at: https://pdxscholar.library.pdx.edu/open_access_etds Let us know how access to this document benefits you.

Recommended Citation

Acres, Colleen Greer, "Spatial clustering of sector linked industry in an urban economy" (1985).

Dissertations and Theses. Paper 356.

https://doi.org/10.15760/etd.356

This Dissertation is brought to you for free and open access. It has been accepted for inclusion in Dissertations and Theses by an authorized administrator of PDXScholar. Please contact us if we can make this document more accessible: pdxscholar@pdx.edu. 


\title{
SPATIAL CLUSTERING OF SECTOR LINKED INDUSTRY \\ IN AN URBAN ECONOMY
}

\author{
by \\ COLLEEN GREER ACRES
}
A dissertation submitted in partial fulfillment of the requirements for the degree of

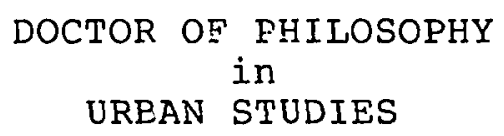


TO THE OFFICE OF GRADUATE STUDIES AND RESEARCH:

The members of the Committee approve the dissertation

of Colleen Greer Acres presented May 23, 1985.

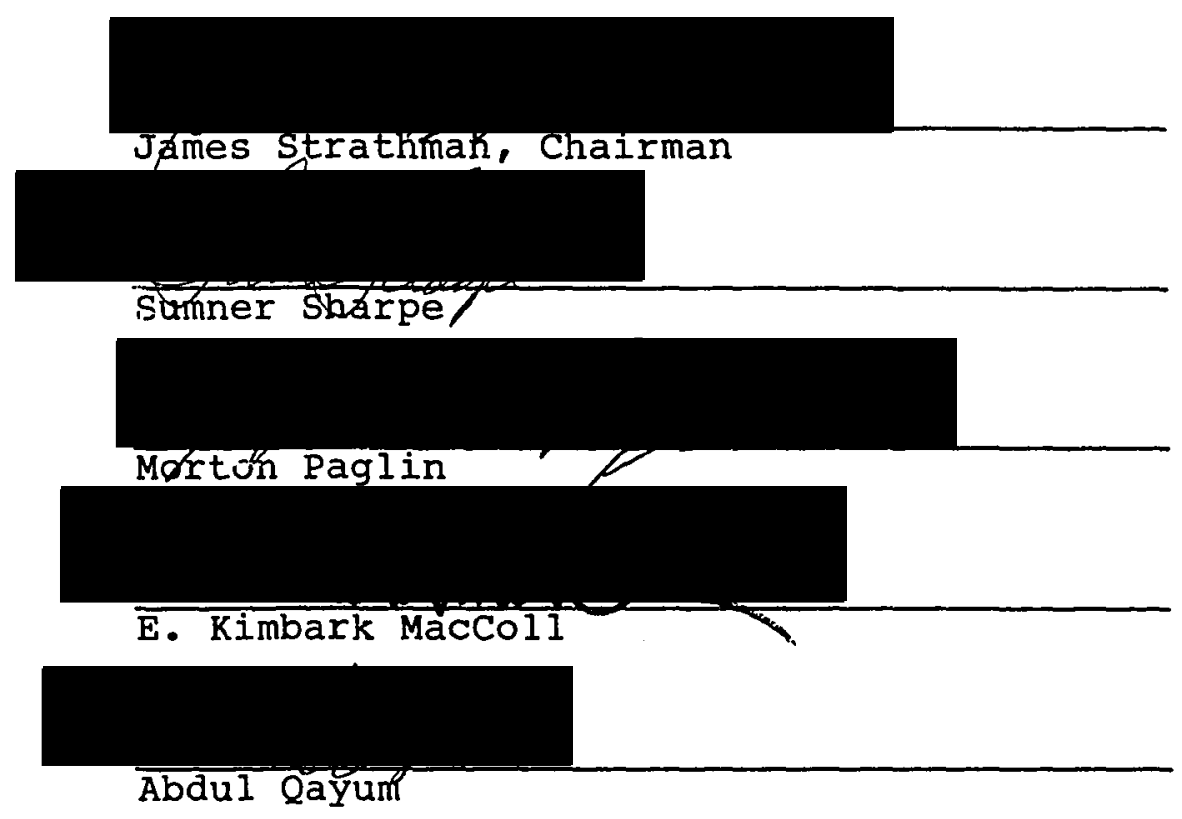

APPROVED:

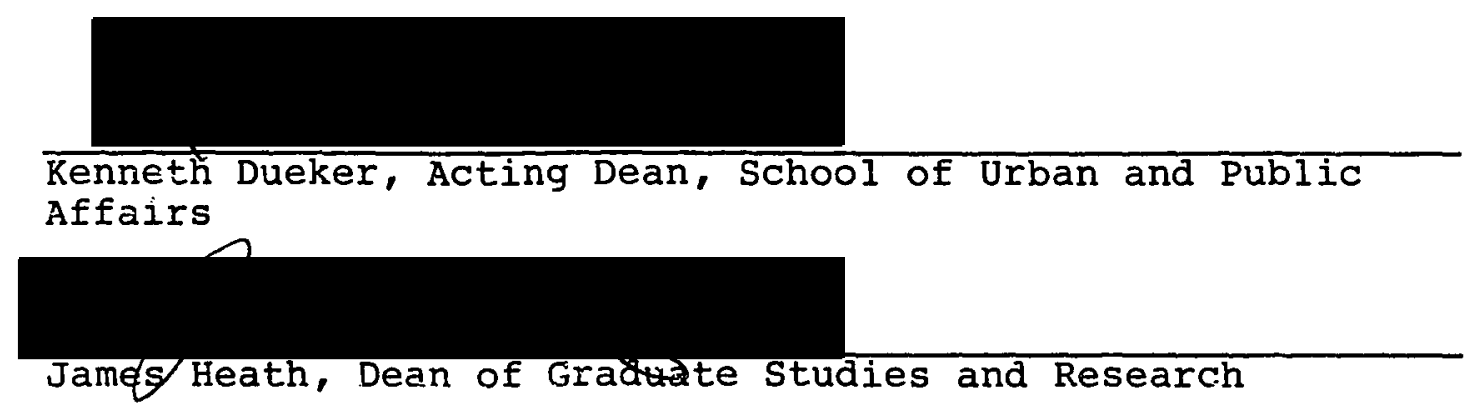




\title{
ACKNOWLEDGEMENTS
}

\author{
Behind this dissertation lie the efforts \\ of a perceptive committee, a sacrificing \\ family, and understanding friends.
}

Thank you all 
TABLE OF CONTENTS

PAGE

ACKNOWLEDGEMENTS . . . . . . . . . . . . . . . iii

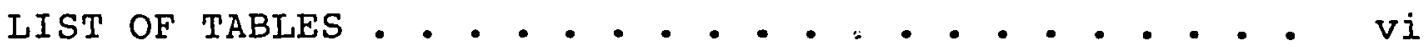

CHAPTER

I INTRODUCTION • . . . . . . . . . . . 1

II REVIEW OF THE IITERATURE • • • • • • • • • 6

Relevant Theoretical Literature . . . 6

Relevant Empirical Literature . . . . 13

Summary of Relevant Literature . . • . 18

III METHODOLOGY . . . . . . . . . . . 20

Conceptual Model . . . . . . . 20

Research Hypotheses. . . . . . . 23

Operational Model . . . . . . . . 24

Measurement of Spatial Proximity . . . 27

Cut-off of Linkage Analysis . . . . 30

Area Selection . . . . . . . . 39

Variable Measurement . . . . . . 45

Spatial Proximity . . . . . . . 45

Linkages ............ 47

Transportation and utility costs . 50

Supply and Market orientation . . 51

Industry Average Firm Size . . . 51

Conclusion . . . . . . . . . 52

IV FINDINGS . . . . . . . . . . . 53

Summary of Regression Findings . . . . 54 


$$
\text { Regression Findings and the Research }
$$




\section{LIST OF TABLES}

TABLE

PAGE

I Spatial Concentration Information . . .

I List of Variables . . . . . . . . .

III Regression of Intra-Industry Linkage With

Covariates Including Manufacturing

Purchases. . . . . . . . . . .

IV Regression of Intra-Industry Linkage With

Covariates Including Manufacturing Sales

$\mathrm{V}$ Regression of First Backward Linkage With Covariates . . . . . . . . . .

VI Regressions of Second Backward and Third

Backward Linkages With Covariates. . .

VII Regressions of Fourth Backward and Fifth

Backward Iinkages With Covariates. . .

VIII Regressions of First and Second Backward and

First, Second, and Third Backward Linkages

with Covariates. . . . . . . . .

IX Regression of First Forward Linkage With

Covariates . . . . . . . . . . .

$\mathrm{X}$ Regressions of Second Forward and Third

Forward Linkages With Covariates. . . .

XI Regressions of Fourth Forward and Fifth

Forward Linkages With Covariates. . . . 
XII Regressions of First and Second Forward and First, Second, and Third Forward Linkages With Covariates . . . . . . . . .

XIII Regressions of One Backward and one Forward

Linkages and One Backward and Two Forward

Linkages With Covariates . . . . . .

XIV Regressions of One Backward and Three Forward

Linkages and Two Backward and One Forward

Iinkages With Covariates . . . . . . 66

XV Regression of Two Backward and Tw' Forward

Linkages and Two Backward and Three Forward

Linkages With Covariates . . . . .

XVI Regressions of Three Backward and One Forward

and Linkages and Three Backward and Two

Forward Linkages With Covariates. . .

XVII Regression of Three Backward and Three Forward

Linkages With Covariates . . . . . 69

XVIII Status of Industries With one Firm . . . 101

XIX Industry Revisions . . . . . . . 103

XX Identification of Manufacturing Industries. $\quad 115$ 
AN ABSTRACT OF THE DISSERTATION OF Colleen Greer Acres for the Doctor of Philosophy in Urban Studies presented May $23,1985$.

Title: Spatial clustering of Sector Iinked Industry in an Urban Economy.

APPROVED BY MEMBERS OF THE DISSERTATION COMMITTEE:
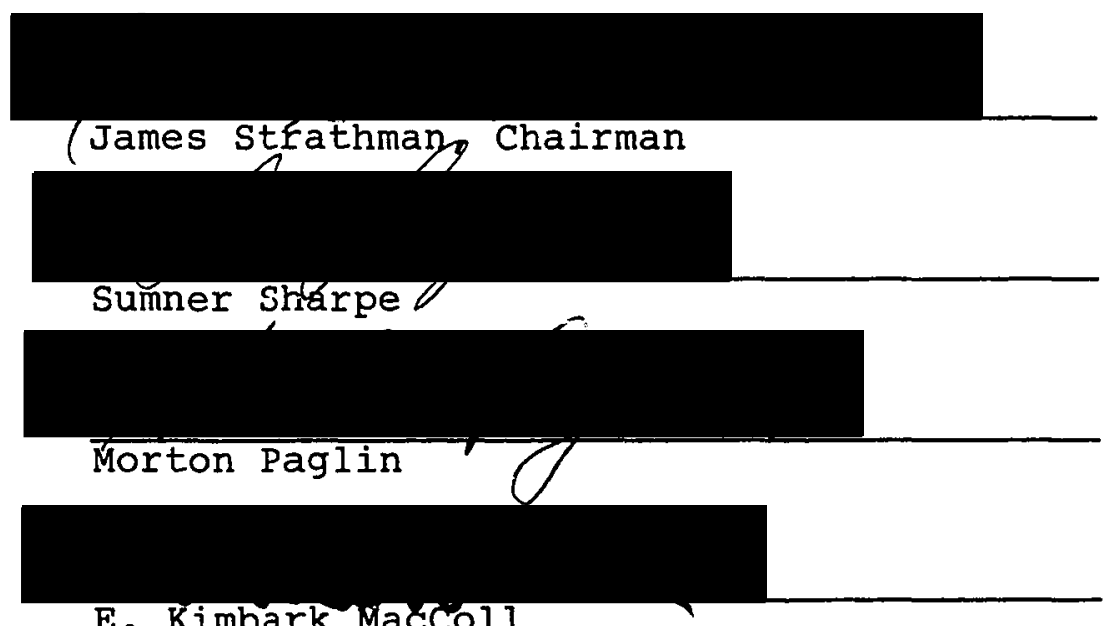

E. Kimbark Maccoll

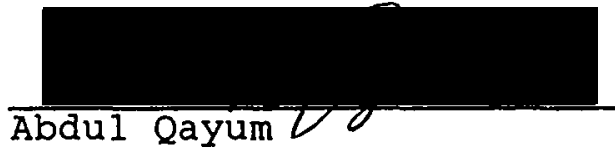

The influence of economies of agglomeration on location decisions has been debated since it was advanced by Alfred Weber in 1909. Empirical findings at the international, national, and regional levels do not definitely support the efficacy of such economies. No study has been done at a local level, the one inherently appropriate to 
the Weber premise. Further, most studies have used highly generalized manufacturing groupings.

The importance of intra-industry and inter-industry determinants of spatial proximity in the Portland, Oregon metropolitan area was investigated in this study. The data base included 220 industries with 2,111 firms employing 108,295 workers. National input-output transaction tables were used to generate measures of intraindustry and inter-industry technological linkage at the 4 digit Standard Industrial Code classification level. Nearest neighbor statistics were employed to measure the spatial proximity of firms within an industry. A spatial association measure, the local concentration coefficient, was devised to calculate spatial proximity among manufacturing firm pairs in various linkage relationships. Then, multiple linear regression was used to examine the relationships of intra- and interindustry linkages to spatial proximity. Average firm size, material and market orientations, and transportation and utility cost intensities were treated as covariates in the analysis. A total of 25 models involving the covariates and various combinations of forward and backward linkages were executed.

In 23 cases, the models and effects of linkages were insignificant. The observed influence of the covariates was generally insignificant. Clearly, 
economies of agglomeration have no effect on industrial patterns in this analysis. Further, the poor performance of the covariates suggest that application of existing theory to localized manufacturing plan selection processes may be misplaced. While these factors may function at the regional or national level, they do not on an intrametropolitan level, at least in this case. Seeking the specific factor of manufacturing linkage based economies of agglomeration or external economies may be a rare exception in location behavior in general and particularly inoperative at a local level. Local development policies which are premised on maximizing such economies are, accordingly, not supported by this research. 
CHAPTER I

INTRODUCTION

The seminal micro-theory of manufacturing location advanced by Alfred Weber (1909) had as a major component the effects of economies of agglomeration on the location decisions of firms. Later theorists such as Hoover (1937, 1948 ) extended this notion to include cost savings associated with close spatial proximity to complementary as well as similar firms under the concept of economies of concentration. For Vernon (1960), Lichtenberg (1960) and Hoover (1967) cost savings achieved by individual firms through their locational choices were referred to as external economies. In contrast, Isard (1960) separated cost reducing factors generated by inter-industry linkages from economies of agglomeration, scale, urbanization, and localization. Regardless of the label applied common to all of these theorists was the contention that spatial proximity to similar firms and transactionally linked firms is an important consideration in the firm site selection process.

Empirical findings have not consistently supported this hypothesis. Alonso (1964) and Smith (1981) found that external economies of agglomeration and inter-industry 
linkages were important determinants of plant location in advanced industrial nations while external economies were more important in underdeveloped nations where firms must compensate for comparatively higher costs of transportation and other factors of production. Conversely, Lausen (1969) in a comparative study of industrial growth patterns in England and the European economic community found that major firms in England were footloose while European counterparts as extensions of existing plants were classified as relatively immobile.

Studies at the regional level have also led to inconclusive results. Inter-regional linkages rather than intra-industry linkages are more important in understanding the behavior of the Pacific Northwest steel industry and manufacturing plant and industry growth in the seattle regional economy according to Schmidt (1973) and Beyers (1975). North (1955), however, attributed the development of the Pacific Northwest to natural resource export market demand. Bell, Lieber, and Rushton (1974) contend that some firms will depart from agglomeration principles when local conditions favor a centrally situated site. Recently Tauchen and witte (1982) note that firm proximity and interaction produce cost savings and revenue generation accruing directly from economies of agglomeration.

Awareness of the impacts of governmental attitudes 
and actions beginning with Hoover in 1940 has been a consistent theme and related subject of debate. The interplay of community political attitudes, community amenities, and executive personal choices in the search for new plant locations was brought into focus by the work of Greenhut in 1963. Holland (1976) advised communities seeking to lessen their vulnerability to economic fluctuations to develop a self-sustaining economic base composed of firms large enough to stimulate linked development through subcontracting and purchasing of materials and components of production. Smith (1981)." on the other hand, tells communities seeking growtti and diversification to create planned growth points supplied with public services necessary to attract industry capable of siting within the community. Tomer (1980) stressed the importance of understanding intra-industry and inter-industry transacticnal patterns in the site selection process regardless of the approach taken by the community. The result has been the development of local economic development programs built on the enhancement of industrial external economies and/or economies of agglomeration.

However, this association between industrial transaction linkage and spatial concentration has never been conclusively substantiated. Even such studies as 
exist are at broad regional levels for grossly aggregated classification levels. The purpose of this investigation was to see if this basically micro-economic concept pertains in micro-circumstances of space and disaggregation. The basic research hypothesis was that spatial proximit: of firms is not related to the magnitude of intra-industry, primary forward and backward, or composite inter-industry linkages. The study was cionducted at a metropolitan geographic scale and industry linkages were disaggregatged to the 4 digit Standard Industrial Classification level.

The Oregon portion of the Portland-Vancouver Standard Metropolitan Statistical Area (SMSA was chosen as the field area for this research. It is varied in topographical features, diverse in economic base and mix of transportation modes, contains a substantial inventory of buildable industrial sites, has available necessary levels of physical and community infrastructure to support industrial growth, and shows a history of supporting industrial growth and diversification. Only recently have studies by the Stanford Research Institute (1982) questioned whether the greater Portland area now contains an adequate inventory of buildable industrial sites capable of meeting the diverse area and site locational requirements of a full range of industries. Confining the 
study to the oregon portion of the metropolitan area limits the heterogeneity produced among subareas by differences created in crossing state political borders.

A covariance application of the multiple regression model was used to test the explanatory power of intraindustry and inter-industry technological linkages in the spatial clustering of manufacturing firms. Spatial proximity, the dependent variable, wasmeasured by the nearest neighbor statistic for firms within an industry. A comparable local concentration measure was developed to calculate spatial arrangements for firms across industries as the nearest neighbor's mathematical properties precluded its use in the latter case. The potential influences of firm size, transportation and utility costs, and supply and market orientation were controlled by their treatment as covariates within the model. The experimental variables--within industry, forward and backward technological linkages-were measured at the 4 digit Standard Industrial Classification level using the 1972 national input-output commodity/industry transactions table. 
CHAPTER I I

REVIEW OF THE LITERATURE

Statements on the influence of intra-industry and inter-industry inkages on the growth and composition of spatial agglomerations are a consistent theme in the development of industrial location theory. Viewed originally as cost minimizing factors, later theorists recognized both cost reducing and/or revenue generating variables in discussions of the determinants of industrial spatial patterns. Lacking in the theoretical literature is a direct and explicit evaluation of the pull which these interdependencies could exert in competition with other determinants of spatial form, e.g. traditional cost variables.

\section{RELEVANT THEORETICAL LITERATURE}

Economies of agglomeration were first defined in 1909 by Weber (1929) as cost savings of firms directly attributable to the presence of similar industries in close spatial proximity. Resultant cost savings were viewed as separate and distinct from economies of scale produced by increasing levels of production or external economies generated by minimizing costs of factors external to production such as transportation. 
The search for other cost minimizing factors continued with the work of Hoover $(1937,1948)$ in his study of the shoe and leather industry and later theoretical formulations. Building on Weber's earlier contributions Hoover noted that cost savings could also be realized by material and market oriented firms locating in close spatial proximity to complementary as well as similar firms. These savings are classified as economies of concentration. Later, Greenhut (1956) extended the analysis of cost and demand factors of location to include revenue-generating as well as cost-reducing factors associated with agglomeration and deglomeration. For example, marketing products or services in an area familiar with a firm's reputation lowers advertising costs relative to competitor cost outlays while increasing sales revenues. Agglomerative economies were seen as a necessary prerequisite for the maximization of possible industrial expansion.

The examination of different input price combinations on areal variations in production costs led Isard (1960) to explain observed firm spatial agglomerations with the use of two types of economies--urbanization and localization. The former arise from vity characteristics of size and composition and clustering of complementary firms. The latter, also referred to as external economies, are achieved by spatial clusters of similar establishments. These are 
viewed as separate and distinct from economies of scale, economies of agglomeration and cost reductions produced by inter-industry linkages. From this classification of economic factors linkages emerge as a competitive determinant whose influence must be independently assessed and then evaluated in conjunction with other factors of location (Isard and Kuenne 1953).

For Perroux (1970) linkage as a concept applied to the economic interdependencies of industries and regions. Growth within a spatially bounded growth pole is attributed to the presence of key industries and inputoutput related non-competitive industries and the occurrence of territorial agglomeration. These active poles in turn induce growth in other areas with relatively passive industries. These latter areas do not possess propulsive industries or the benefits of agglomeration. Input-output industry transactions are recognized as one of the primary factors in the economic growth of regions and nations. Inter-industry linkages are seen as increasingly important by Stabler (1968) as the number of stages in the production process grows and the number of industries directly connected to natural resource inputs or final markets decreases. The establishment and growth of industrial complexes is attributed to industries seeking to minimize inter-industry transfer costs and maximize 
accessibility to both suppliers and buyers.

Rather than being industry wide Romanoff (1984) argues that a need for spatial proximity is tied to the nature of an industry's production process. Manufacturers who produce goods in response to orders are seen as more dependent upon close contact with markets than those who who produce goods for mass consumption and in anticipation of demand.

Other theorists use firm size and/or corporate organizational structure to account for differences in economically linked inter-industry spatial patterns. Small firms are characterized as more vulnerable to external market forces (Galbraith 1975; Holland 1976). At the same time these firms are also more likely to be dependent upon the local economy for their orders (O'Farrell 1985). Therefore a location in close proximity to trading partners is used to minimize market uncertainities and increase accessibility to trading partners (Moomaw 1985). Conversely, large firms are seen as having the power necessary to control their environment. If not, an option open to large firms is the vertical integration of product stages as cost factors subject to economies of scale are internalized (riakanson 1979).

The influence of inter-industry linkages, firm size, and other factors in the selection of plant location 
was examined by the above theorists within varying spatial scales ranging from the micro to macro levels of development. While distinctions were generally made between areas the applicability of the variables to the selection of a specific site among alternatives within an area was not addressed.

The selection of a plant location is a two tier decision-making process according to Greenhut (1964). Distinction is made between demand as an area-determining and site determining locational factor. While market proximity is a factor in the selection of an area locations of competitors and interdependence of firms are seen as the operational aspects of demand at the site level.

A similar but more inconclusive distinction is made by Thomas (1975) in the typology of environments within which the firm conducts business and decisions are made. Constraints and opportunities for growth are seen as influenced by the characteristics of the firm, the industry, the geographic area, and the socio-economic-political system. Adding to this base are the works of network and informational theorists who challenge the economic assumption of complete knowledge and suggest that a firm is further constrained by the a firm's planning space, informational space, and decision space (Taylor 1979; 
Moses and Williamson 1967; Mathur 1981; Kipnis 1984).

The integration of these two viewpoints raises the question of whether equivalence exists between the definition of space as used by the researcher and that used by the individual firm or industry in the site selection process. Clearly, there is a need for the examination of firm interviews or other primary data a comprehensive theory of the plant location selection process can be developed. Finally, no treatment of interdependencies among input/output linked firms and industries could be considered complete without a discussion of transportation and inventory factors. Isard (1951) called attention to the the substitution principle whereby labor or transportation cost savings could be used to counterbalance increased costs of other inputs at a given location. Hakimi (1964) in his modeling of transportation factors suggests that the optimal plant location will be at the market. Iouveaux, Thisse, and Beguin (1982) argue that the optimal location for plant location will be at inter-modal transshipment nodes rather than at the market unless large fixed transportation costs are evident. Even here, a market location may not be the optimal location if, according to these authors, the firm is dependent upon a number of markets. Inventory costs can be treated in the same way as transportation costs according to Louveau etal 
(1982). The second and third theorems are viewed as applicable to the determination of the effect of inventory costs and management practices on plant location choices. Empirical studies in the next section of this chapter examine the importance of transportation costs in the site selection process within metropolitan areas. Each of the theorists reviewed above investigated the possible influence transportation costs within a regional context or, as in the case of Isacd, at the individual firm level. No agreement has been reached at the theoretical level.

To sum, Weber (1909) called attention to the notion that cost savings could be generated by locating in close spatial proximity to similar firms. Hoover (1937) extended this premise to include cost savings generated by firms locating in close spatial proximity to complementary firms. These savings were variously referred to as economies of agglomeration (Weber 1929), concentration (Hoover 1937), territorial agglomeration (Perroux 1970), external economies (Vernon 1960), and demand factors of location (Greenhut 1964). For other theorists, interindustry linkages are a competitive factor in the plant selection process and distinct from each of the above economies (Isard 1951). Mitigating the influence of inter-industry linkage on firm spatial proximity are the characteristics of firms, industries, geographic 
areas, and socio-economic-political systems according to Thomas (1975). Need to differentiate area and site determining factors is suggested by Greenhut (1964).

In each of these cases, inter-industry linkages receive secondary treatment as a component within a broader theoretical framework. Trade relationships among firms within an industry are ignored entirely. It can be argued, however, that inter-industry linkages include intra-industry linkages as the latter are an artifact of the use of highly aggregated industrial categories. By this argument intraindustry linkages become a surrogate for a set of linkages which are beyond the precision of a given transactions table and common definitions pertain.

This lack of theoretical focus suggests that either inter-industry linkages are a minor locational factor or that their influence may be easily diluted by other locational factors. However, there is no concensus about the importance of other locational factors such as labor and transportation costs within a regional economy. The purpose of this study is to directly examine the influence of input-output linkages on spatial proximity of manufacturing firms within an urban esonomy.

RELEVANT EMPIRICAL LITERATURE

Empirical studies can be classified according to 
whether their focus is on the the existence of linkages or the influence of linkages on the attractiveness of an area for growth and diversification. These studies may be international, national, regional, or local in their scope. While findings are able to document the presence of linkages they do not definitively support postulates which associate linkages with area attractiveness or the spatial proximity of similar or complementary firms. Lausen in his comparative study of Britain and the European Economic Community found that firm site choices could not be explained by inter-industry linkage arrangements. Manufacturing firms in England were found to be relatively footioose. As extensions of existing manufacturing facilities new European plants, on the other hand, were found to be relatively immobile. Similar results were found by Peschel (1982) in the study of European economic integration. The presence of agglomeration areas with clusters of linked industries were not found to help or hurt the attraction of new firms or to reduce opportunities for growth at the periphery. Auty (1975) in a study of sugar industries in Carribean countries found that plant size was the critical factor in the relationships which firms established with other firms. Small firms were also seen as embodying different technologies than large firms. 
Streit (1969) found weak relationships between spatial association and economic alliance in his study of industries in the United Kingdom. Linkages were not found to be a binding force among transactionally related firms. Similar results were found by Harrigan (1982) in a study of industrial linkages and distributions in England using clustering algorithms designed to generate industrial and spatial clusters. External economies based on information/communication and amenities were used to explain concentrations of high technology industries. For others such as aerospace, timber and lumber, and shipbuilding labor costs and/or port proximity were used to account for spatial clusters identified in the study. Comparable results were found by Czamanski and Czamanski (1977) in their study of manufacturing clusters in the United States. Conversely, strong local linkages were found in a study of metal industries in West Midlands, England by Taylor and Wood (1975). These linkages were found to be important to small firms with less than 50 employees but not for plants with 90 or more employees. The presence of linkages was confirmed by O'Farrel1 (1985) in the study of surviving plants in Ireland. However, the presence of intra-national linkages which tied firms to domestic demand slowed the growth of plants relative to that observed in export linked firms. 
The driving force for the growth of steel industries in the Pacific Northwest was attributed to inter-regional or export linkages by schmidt (1973). In this same region Beyers (1975) in a study of Puget Sound, Washington as a growth center found weak linkages between industries within the region. In studies of Joinville, Brazil and 12 growth centers in Israel, Kipnis (1984) found the strongest within area linkages among small and medium firms. Multiplier effects could be traced to the growth of these firms and their forward linkages in particular. Among large plants only textile industries were found to have relatively strong local linkages.

Vernon in his New York Study (1960) found that spatial proximity was more important to small firms taking advantage of external economies generated by sharing the costs of support services. The clustering of firms within the central city was attributed to firm needs for communication/information, reduction in the costs of uncertainity, and opportunities to take advantage of economies not inter-industry linkages. No significant differences were observed between the central city and outlying areas on available space, transportation costs, or taxes.

Birch (1970)) in a comparative study of American metropolitan areas found that manufacturing firms were 
moving to the suburbs. Linkages could not be used to explain their relocations. The attraction of manufacturing firms to the young cities was attributed to the availability of large open spaces and relatively new transportation systems. However, a recent study by Walker (1977) in England using analysis of variance found that spatial clusters of industries and complementary activities existed and tilat inter-sectoral linkages were important to the formation and growth of these spatial concentrations. Less important were the inter-industry linkages within manufacturing.

These apparent contradictions in the literature are attributed by wood (1969) in his study of industrial location and linkages as the inadequacy of traditional theory which "...regards as awkward exceptions the complex agglomerations that are supposed to be based on principles of external economies of scale and close functional linkage." More than ten years later Smith (1981) following a comprehensive review of industrial location theory and research concludes that economies of agglomeration and inter-industry linkages will become more important in the future for industries and firms within advanced industrial societies. Present problems with the concepts are dismissed essentially as problems of measurement. 
SUMMARY OF RELEVANT LITERATURE

Intra-industry and inter-industry linkages represent the transactional relationships which firms establish through their purchase of inputs (backward linkages) and sale of outputs (forward linkages). Plant location theory postulates that cost-reducing and revenue-generating opportunities exist for firms who locate in close spatial proximity to suppliers and markets. Economic development practitioners use these concepts to shape community development and target industry programs at each level of government. While the desired network of new firms and economic opportunities include all sectors of the economy the emphasis is usually placed upon the manufacturing industries as drivers of the economy which will create the largest impacts through the basic multiplier effect.

In actuality, evidence on manufacturing linkage impacts at the regional, national, and international levels does not substantiate the importance of linkages at the metropolitan or community level. Nor does research which focuses on the total economy, manufacturing industry serving, or consumer oriented sectors test the attributed ability of manufacturing linkages to influence the spatial patterns of production.

Assuming that a decision has been made to build a 
new facility rather than utilize other options available such as expansion of an existing plant, choices must be made between competing areas and sites. The existing industrial $\mathrm{mix}$ is a product of characteristics of both area and site environs. If technological linkages are important locational determinants for firms, clustering should be observable in the landscape as linked firms choose locations which minimize distance with respect to the strength of linkages. 
METHODOLOGY

This research tested hypotheses about the influence of linkages as determinants of close spatial proximity within a local urban ecoromy. Variability in locational concentration patterns associated with other factors was controlled for by the use of a covariance model of multiple regression. Formalization of the research hypotheses, research ciesign, area definition, measurement, and data collection are addressed below.

\section{CONCEPTUAL MODEL}

The patterns of spatial proximity among firms in the urban economy can be seen as a function of the strength of intra-industry and inter-industry linkages, factor of supply and market orientation, firm size, transportation costs, and utility costs. Stated as a conceptual model:

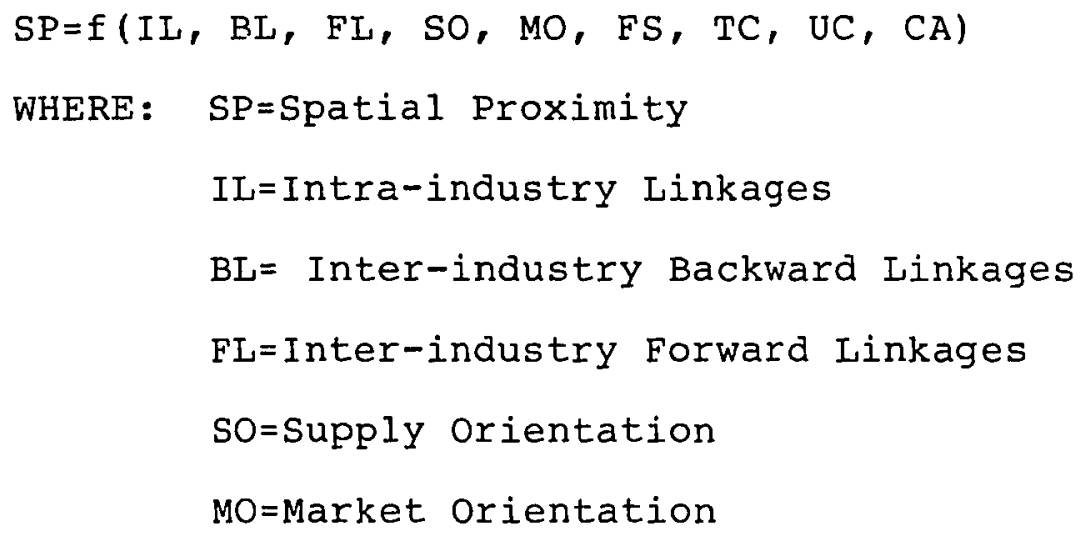




\author{
$\mathrm{FS}=\mathrm{Firm}$ Size \\ TC=Transportation costs \\ uC=Utility Costs \\ $\mathrm{CA}=\mathrm{A}$ Vector of Community Attributes
}

Firms differ in the number and magnitude of their transactional relationships with similar firms (IL) and other manufacturing firms (BL/FL). Trade relationships may be occasional, temporary, or routine. It has been assumed that in genoral the greater the frequency and magnitude of exchanges among given firms the more likely close spatial proximity (SP) will produce cost reducing savings and/or revenue generation at levels considered important to the firm. Spatial proximity (SP) is said to facilitate the timely exchange of goods and services, the possibility for the externalization of inventory costs (Samuelson 1957; Louveau, Thisse, and Beguin 1982), and establishment and development of informational flows and relationships (Moses and Williamson 1967 ) and the minimization of uncertainity (Webber 1972). The validity of these premises at the metropolitan level is questionable where limited transportation costs are involved.

The pull exerted by suppliers or markets will depend upon the types of inputs required in the production process (SP), the nature and composition of firm markets (MO), and the transportation costs (TC) involved in the movement of 
goods and services. Obviously the greater the areal variation in these factors both individually and in combination, the steeper will be the cost and revenue surfaces and the more likely firms will be to seek locations in close proximity to firms which provide the factors to which they are most cost or revenue sensitive. The sensitivity of firms to these factors will also be dependent upon the size of the firm (FS), with smaller firms having more limited sets of options.

Traditional factors of producticn--land, labor, and capital--may constrain or enhance the availability of locational choices (Smith, 1981). The availability of an iventory of buildable industrially zoned sites supplied with adequate types and levels of social and physical infrastructure is necessary for the community seeking economic growth and diversification. Availability and costs of delivered services (UC) such as electricity, gas, water, and sanitary sewer and appropriate transportation modes for freight and passenger movement (TC)-roads, railroad, air, water, and pipeline-may be critical factors in the selection of areas and sites. Social infrastructure availability and cost is likely to be important to firm owners, executives, and line workers as costs will directly impact both the firm and its labor force.

Finally, the attitudes, policies, and programs of the community may increase or decrease area attractiveness. 
The pursuit of business and industry is a highly competitive process (CEDO 1983). Public infrastructure and plant investments are the result of decisions with long term consequences for the community and the industry. Community attitudes, politics, and programs are a barometer of the receptivity of the community to the industry both now and in the future. As such they bear directly on the present and future costs of doing business in the area and at that site in a longitudinal framework. Unlike other costs previously mentioned, many of the variables important to the assessment of the community may not be amenable to quantification in monetary terms. But, the importance of this evaluation is well substantiated in the literature and the practical experience of communities seeking economic development. Through study design, variable selection and measurement, and use of statistical procedures address each of the elements presented in the conceptual model as elaborated here must be addressed.

\section{RESEARCH HYPOTHESES}

Derivable from the above discussion are statements which describe the hypothesized types and directions of relationships expected among identified variables. Current practice, which has accepted intrinsically micro level theoretical statements and inferred empirical findings at the macro level about the importance of linkages at the 
metropolitan level. This research directly addresses the influence of linkages in a metropolitan setting. The research hypotheses are:

1. The spatial proximity of firms in an industry is not related to the magnitude of intra-industry technological linkages.

2. The spatial proximity of firms is not related to the magnitude of their primary forward and backward linkages.

3. The spatial proximity of firms is not related to the magnitude of their composite forward and backward linkages.

The expected aggregate impact of firm size, supply and market orientation, transportation costs, and utility costs will be controlled by the use of a covariance application of multiple regression. The extraction of the variability in the dependent variable, spatial proximity, with these covariates will allow the measurement of effects directly attributable to the active or experimental variables-intra-industry and inter-industry linkages.

\section{OPERATIONAL MODEL}

The model takes the following linear form:

$$
\mathrm{Y}=\mathrm{a}+\mathrm{b}_{1} \mathrm{X}_{\mathrm{Cl}}+\ldots+\mathrm{b}_{\mathrm{k}} \mathrm{X}_{\mathrm{CK}}+\mathrm{b} \mathrm{X}_{\mathrm{E} 1}+\mathrm{e}
$$


WHERE $a, b_{i}=$ empirically estimatea parameters

$$
\begin{aligned}
& \mathrm{x}_{\mathrm{C} 1} \ldots \mathrm{x}_{\mathrm{CK}}=\text { Covariates } \\
& \mathrm{X}_{\mathrm{E} 1}=\text { Experimental Variable } \\
& \text { e=Error Term }
\end{aligned}
$$

Independent variables identified as covariate candidates include firm size, supply and market orientation, transportation costs, utility costs, and community attributes. The former will be included in the model as covariates. The latter, as discussed earlier, are not readily amenable to quantification. Variability in the dependent variable attributable to differences among area geographic subunits will be controlled by the selection of an area where differences among geographic subunits are minimized.

Intra-industry and inter-industry linkages are the experimental variables in the design. The contribution of each linkage to variability in the dependent variable will be examined individually and in linear combination. No relationship is expected between the experimental variables, intra- and inter-industry linkages and the dependent variable. In order to test the research hypotheses the regression model will take the following general form:

$$
\begin{aligned}
\mathrm{Y}_{I J K}=a+ & b_{1 I F} x_{1 F}+b_{2 I S} x_{2 I S}+b_{3 I M} x_{3 I M} \\
& +b_{4 I T} x_{4 I T}+b_{5 I U} x_{5 I U}+b_{6 I J K} x_{6 I J K} \\
& \text { iE }
\end{aligned}
$$


WHERE: $\mathrm{Y}_{\mathrm{IJK}}=\mathrm{R}_{\mathrm{NIJK}}$ and $\mathrm{R}_{\mathrm{NIN}}=2 \mathrm{~d}_{\text {IJK }} \mathrm{N}_{\mathrm{R}} \mathrm{IJK} \quad$ (Clark and Evans 1954)
WHERE:

$d_{I J K}=$ the average of the nearest neighbor distances for each firm in industry I

$\mathrm{N}_{\text {IN }}=$ the number of firms in industry $\mathrm{I}$

$A_{R}=$ the area of the region under investigation

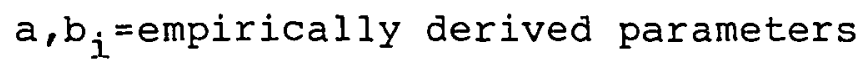

$\mathrm{F}_{1 \mathrm{IF}} \ldots \mathrm{X}_{5 I \mathrm{U}}$ are covariates

and

$\mathrm{X}_{\text {IF }}=$ industry $\mathrm{I}$ average firm size

$\mathrm{X}_{2 \mathrm{~S}}=$ manufacturing purchases as a percentage

of total purchases in the industry

$\mathrm{X}_{3 \mathrm{M}}=$ manufacturing sales as a percentage

of total industry sales

$\mathrm{X}_{4 \mathrm{IT}}=$ transportation purchases as a percentage

of total industry purchases

$\mathrm{X}_{5 I U}=u t i l i t y$ purchases as a percentage

of total industry purchases

$\mathrm{X}_{6 \mathrm{IJK}}=$ percent of intra-industry purchases

among all firms in industry $I$ to

total manufacturing purchases

Consideration of the variability in inter-industry spatial proximity attributable to an inter-industry backward 
or forward linkage results in substitutions in the above equation for the dependent and experimental variables. Combinations are treated in similar fashion.

\section{MEASUREMENT OF SPATIAL PROXIMITY}

Spatial proximity of firms within an industry is measured by the use of nearest neighbor analysis. Spatial proximity of subject industry firms to forward and backward linkage firms is measured by the use of a new and comparable technique devised to work with inter-industry linkages--the local concentration coefficient.

Developed by Clark and Evans (1954) the nearest neighbor technique calculates the mean of the distances between all firms within an industry and their nearest neighbor. Divided by the expected mean nearest neighbor distance for a random arrangement the index provides a concise measure of pattern in terms of a single value (Ebdon 1981). The index can take a value between 0.0 (a clustered pattern) and 2.12 (a dispersed pattern). A value of 1.00 indicates a random arrangement of points. Criticisms of nearest neighbor analysis have focused on the sensitivity of the index as a direct statistic to differences in the shape of a bounded region under investigation (Hsu and Tiedemann 1968) and modifications made to the two-dimensional Poisson model in its application to the nearest neighbor analysis (Ingram 1978). Hsu and 
Tiedemann (1968) using a hypothetical metropolitan area as an example that a pattern identified as random within the central city needed to be reclassified as clustered in the area of measurement included the surrounding suburban and urban fringe areas. Later Hsu and Mason (1972) suggested that this problem could be handled by development of alternative probability models.

These are reasonable concerns for its use as a statistic. However, in this investigation nearest neighbor is used only as a measurement of point array. All scores are based on the same geographical unit.

Thus the design of this research, which focuses on a bounded urban area with an integrated economic base and a fixed size and shape, circumvents the need to consider alternatives to the measurement of points in a region originally suggested by Clark and Evans (1954).

Continued use of nearest neighbor analysis seems, at first glance, appropriate. However, this technique requires the use of all points within the plane. Matching of interindustry nearest neighbor pairs means that all subject firms as oxigins or destinations of goods will be included in the analysis. But linkage firms exist on a separate plan and will be exluded from the analysis if they are not the nearest neighbor of their type to subject firms and some linked firms. Therefore, the nearest neighbor technique cannot be used. 
Cluster algorithms as used by Harrigan (1982) to identify integrated industry spatial clusters or by walker (1977) to determine general spatial associations among establishment groupings do not measure nearest neighbor pair distances. What is needed is a measure which is comparable in formulation to the nearest neighbor technique and results in the a single index of the correlation among given pairs of points. A measurement called the Local Concentration Coefficient (LC) is advanced to measure of general spatial proximity of linked pairs.

The IC calculates the average length of lines between point pairs in the 2 industry pattern and compares this to the longest distance that could be expected to occur between 2 linked firms within the oregon portion of the PortlandVancouver SMSA.

Stated formally:

$$
{ }^{k l_{I C}} C_{I I J}=\sum_{n B} b_{i j}
$$

WHERE: $b_{i j}=$ the distance from the ith firm of the kth industry to its nearest firm, $j$, of linked industry 1 . $\mathrm{n}=$ the number of ith firms $B=$ the greatest possible distance from an ith firm to an lth partner.

The use of a referent line (B) in the equation's denominator which is the longest distance that could be 
expected between any two linked firms in the oregon portion of the Portland-Vancouver SMSA establishes a benchmark against which measured distances among pairs can be contrasted. The measure ranges from 0.0 indicating complete clustering to 1.00 which indicates a completely dispersed pattern of points within the bounded area. Again, both the nearest neighbor technique and the local concentration coefficient are used here strictly as measures of the dependent variable, spatial proximity. Industry scores are presented in Table I to allow an inspection of concentration patterns.

\section{CUT-OFF OF IINKAGE ANALYSIS}

Linkage data are expressed as percentages of total purchases and sales from a given kth base industry. All industries which supplied 5 percent or more of the value of the value of total manufacturing sales were included in the analysis. This resulted in the identification of up to 8 backward and 9 forward linkages for a given base $(k)$. LC's for all paired patterns up to 17 for a single base industry were calculated. However, there were so few industries with more than 5 backward or forward interindustry linkages that the analysis of 6 th, 7 th, 8 th, and 9 th inter-industry linkages was technically impractical by multiple linear regression.

Combinatorial concentrations were represented by 
TABLE I

SPATIAL CONCENTRATION INFORMATION

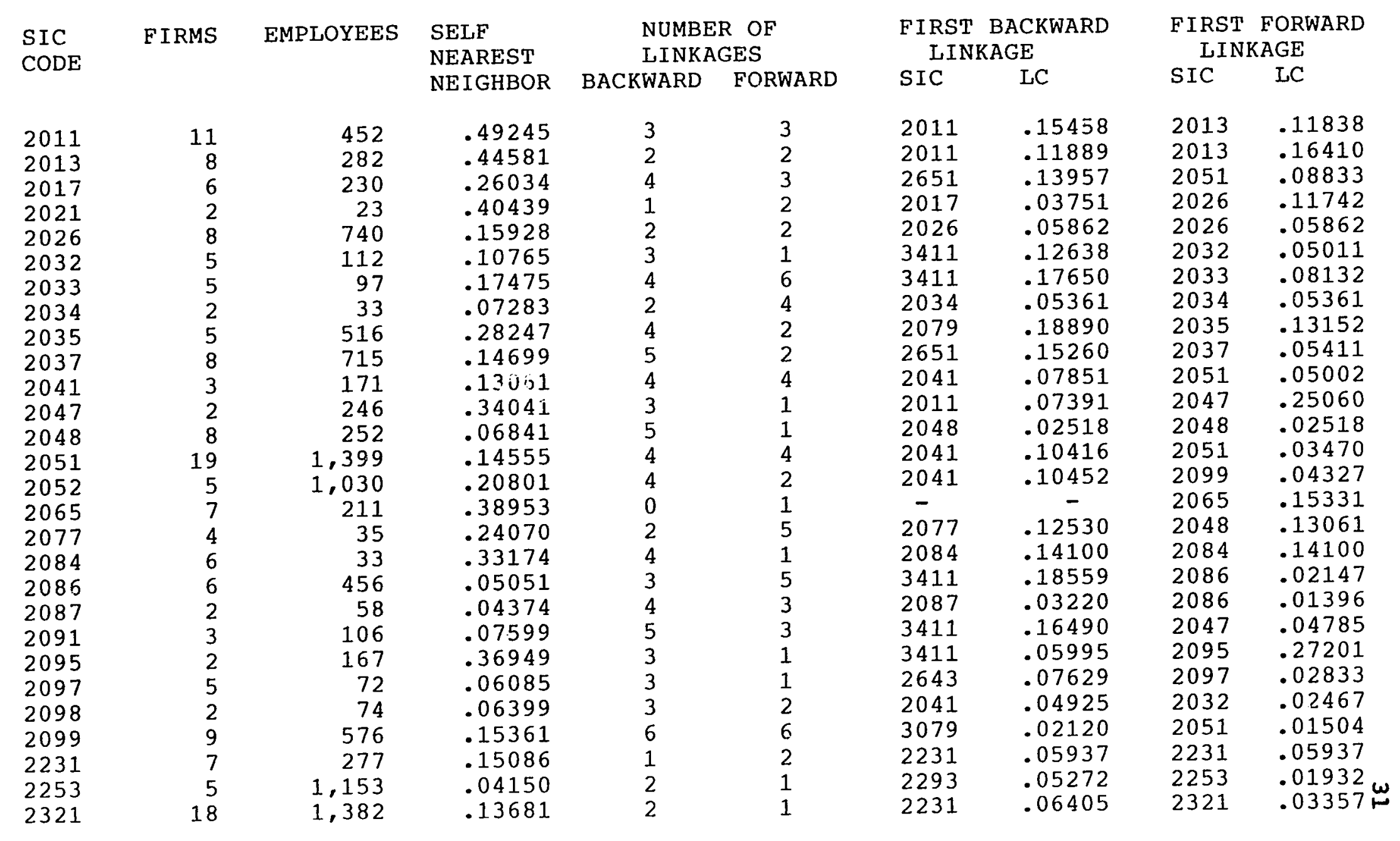


TABLE I (CON'T)

SIC
CODE

2391
2392
2393
2394
2399
2411
2421
2426
2429
2431
2434
2435
2439
2448
2452
2491
2499
2511
2512
2515
2521
2531
2541
2542
2591
2599
2621
2641
2642
2643

FIRMS EMPLOYEES

SELF

NEAREST

NEIGHBOR

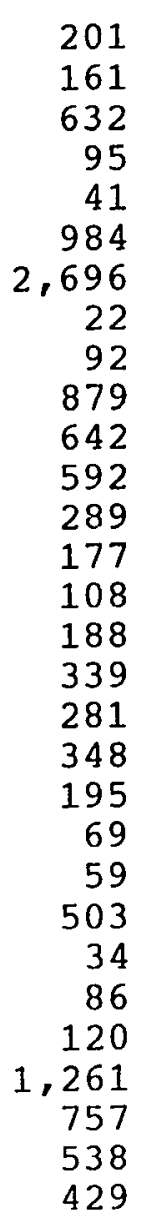

NUMBER OF LINKAGES BACKWARD FORWARD
FIRST BACKWARD LINKAGE SIC

2231

2231

2299

2293

2231

3496

2411

2421

2411

2421

2435

2411

2421

2421

2421

2421

2421

2421

2231

3496

2421

3312

3079

3312

3079

3312

2411

2621

2621

2621

LC

.07599
.05831
.13148
.06024
.06715
.35751
.05816
.01722
.07716
.03315
.10115
.04287
.03768
.04342
.04122
.05660
.03558
.04114
.12948
.04947
.02792
.20024
.02876
.10492
.00774
.16918
.05146
.08941
.08639
.04300

FIRST FORWARD LINKAGE

SIC LC

$\begin{array}{ll}2391 & .04025 \\ 2392 & .07026 \\ 2041 & .11055 \\ 3732 & .05118 \\ 3711 & .05503 \\ 2421 & .05503 \\ 2431 & .10717 \\ 2511 & .07717 \\ 2499 & .19610 \\ 2452 & .06803 \\ 2431 & .06373 \\ 2435 & .11951 \\ 3732 & .07040 \\ 3079 & .05972 \\ 2452 & .12282 \\ 3079 & .03128 \\ 2499 & .07941 \\ 3792 & .05272 \\ 3792 & .10865 \\ 2515 & .04637 \\ 2521 & .10786 \\ 3711 & .20880 \\ 3732 & .06365 \\ 3312 & .10492 \\ 2591 & .01247 \\ 3731 & .09318 \\ 2752 & .03477 \\ 2751 & .03430 \\ 2721 & .02855 \omega \\ 2051 & .05827 \omega\end{array}$


TABLE I (CON'T)

\begin{tabular}{|c|c|c|c|c|c|c|c|c|c|}
\hline \multirow[t]{2}{*}{$\begin{array}{l}\text { SIC } \\
\text { CODE }\end{array}$} & \multirow[t]{2}{*}{ FIRMS } & \multirow[t]{2}{*}{ EMPLOYEES } & \multirow{2}{*}{$\begin{array}{l}\text { SELF } \\
\text { NEAREST } \\
\text { NEIGHBOR }\end{array}$} & \multicolumn{2}{|c|}{$\begin{array}{l}\text { NUMBER OF } \\
\text { LINKAGES }\end{array}$} & \multicolumn{2}{|c|}{$\begin{array}{l}\text { FIRST BACKWARD } \\
\text { LINKAGE }\end{array}$} & \multicolumn{2}{|c|}{$\begin{array}{l}\text { FIRST FORWARD } \\
\text { LINKAGE }\end{array}$} \\
\hline & & & & BACKWARD & FORWARD & SIC & LC & SIC & LC \\
\hline 2646 & 2 & 62 & .67198 & 2 & 1 & 3079 & .04822 & 3312 & .24949 \\
\hline 2649 & 3 & 87 & .19473 & 3 & 4 & 2621 & .07714 & 3079 & .05316 \\
\hline 2651 & 11 & 716 & .09192 & 1 & 1 & 2621 & .05533 & 2026 & .05103 \\
\hline 2711 & 32 & 2,115 & .25970 & 2 & 1 & 2621 & .10442 & 2711 & .04780 \\
\hline 2721 & 20 & 110 & .25642 & 2 & 2 & 2751 & .01986 & 2711 & .02305 \\
\hline 2731 & 9 & 51 & .12087 & 3 & 1 & 2621 & .05761 & 2731 & .04195 \\
\hline 2741 & 8 & 53 & .12448 & 3 & 4 & 2789 & .05076 & 2741 & .04582 \\
\hline 2751 & 171 & 1,878 & .13610 & 4 & 4 & 2621 & .06505 & 2721 & .03541 \\
\hline 2753 & 5 & 110 & .13708 & 3 & 3 & 2621 & .06084 & 2752 & .00394 \\
\hline 2761 & 7 & 258 & .22785 & 3 & 2 & 2621 & .07987 & 3622 & .06827 \\
\hline 2782 & 7 & 390 & .12434 & 3 & 1 & 2721 & .04806 & 2782 & .05601 \\
\hline 2789 & 6 & 101 & .06818 & 3 & 4 & 2621 & .06818 & 2731 & .02644 \\
\hline 2791 & 23 & 141 & .11301 & 2 & 4 & 3861 & .04894 & 2751 & .00630 \\
\hline 2793 & 5 & 54 & .05805 & 4 & 1 & 3356 & .04498 & 2751 & .00391 \\
\hline 2795 & 5 & 53 & .03414 & 6 & 2 & 3354 & .03429 & 2751 & .00305 \\
\hline 2812 & 7 & 608 & .16255 & 1 & 4 & 2812 & .06397 & 2812 & .06397 \\
\hline 2821 & 4 & 81 & .08365 & 3 & 2 & 2812 & .02518 & 3079 & .03803 \\
\hline 2831 & 12 & 330 & .21577 & 5 & 2 & 2831 & .06485 & 2831 & .06485 \\
\hline 2841 & 5 & 80 & .12884 & 3 & 2 & 2812 & .05701 & 2011 & .14049 \\
\hline 2842 & 7 & 86 & .20378 & 5 & 2 & 2819 & .05694 & 2842 & .08019 \\
\hline 2851 & 12 & 324 & .20679 & 3 & 4 & 2812 & .06803 & 3711 & .10388 \\
\hline 2875 & 2 & 14 & .42766 & 2 & 1 & 2873 & .30404 & 2875 & .31483 \\
\hline 2879 & 7 & 275 & .21000 & 3 & 3 & 2812 & .06272 & 2879 & .08264 \\
\hline 2891 & 6 & 133 & .12849 & 4 & 5 & 2812 & .04037 & 3714 & .06429 \\
\hline 2893 & 7 & 99 & .16100 & 4 & 4 & 2812 & .04588 & 2751 & .01348 \\
\hline 2899 & 5 & 133 & .09028 & 5 & 2 & 2812 & .02662 & 2851 & .01888 \\
\hline 2911 & 5 & 84 & .11236 & 2 & 4 & 2911 & .05234 & 2991 & .05234 \\
\hline 2952 & 6 & 490 & .09739 & 2 & 2 & 2911 & .02868 & 3711 & .04856 \\
\hline 3011 & 2 & 80 & .00023 & 1 & 2 & 2819 & .01936 & 3711 & $.04711 \underset{\omega}{\omega}$ \\
\hline 3041 & 3 & 30 & .06545 & 3 & 1 & 2262 & .02575 & 3711 & م 05499 \\
\hline
\end{tabular}


TABLE I (CON'T)

\begin{tabular}{|c|c|c|c|c|c|c|c|c|c|}
\hline \multirow{2}{*}{$\begin{array}{l}\text { SIC } \\
\text { CODE }\end{array}$} & \multirow[t]{2}{*}{ FIRMS } & \multirow[t]{2}{*}{ EMPLOYEES } & \multirow{2}{*}{$\begin{array}{l}\text { SELF } \\
\text { NEAREST } \\
\text { NEIGHBOR }\end{array}$} & \multicolumn{2}{|c|}{$\begin{array}{l}\text { NUMBER OF } \\
\text { LINKAGES }\end{array}$} & \multicolumn{2}{|c|}{$\begin{array}{l}\text { FIRST BACKWARD } \\
\text { IINKAGE }\end{array}$} & \multicolumn{2}{|c|}{$\begin{array}{l}\text { FIRST FORWARD } \\
\text { LINKAGE }\end{array}$} \\
\hline & & & & BACKWARD & FORWARD & SIC & LC & SIC & LC \\
\hline 3069 & 7 & 409 & .23941 & 4 & 3 & 2812 & .09394 & 3711 & .15267 \\
\hline 3079 & 55 & 1,386 & .21772 & 4 & 1 & 2821 & .19220 & 3079 & .03056 \\
\hline 3111 & 2 & 50 & .41882 & 3 & 3 & 2011 & .06535 & 3143 & .14671 \\
\hline 3143 & 2 & 55 & .20359 & 3 & 1 & 3111 & .08890 & 3143 & .14988 \\
\hline 3151 & 2 & 37 & .00303 & 2 & 0 & 3111 & .00223 & - & - \\
\hline 3199 & 6 & 80 & .13870 & 5 & 4 & 3111 & .07997 & 3143 & .04608 \\
\hline 3221 & 2 & 538 & .07810 & 4 & 6 & 2651 & .06218 & 2086 & .12427 \\
\hline 3211 & 5 & 84 & .24656 & 4 & 3 & 3211 & .11480 & 3711 & .15762 \\
\hline 3241 & 2 & 192 & .18917 & 4 & 3 & 2812 & .05139 & 3273 & .01499 \\
\hline 3251 & 3 & 68 & .84076 & 5 & 0 & 3499 & .18060 & - & - \\
\hline 3259 & 2 & 16 & .36367 & 6 & 1 & 2421 & .11888 & 3259 & .26773 \\
\hline 3269 & 5 & 126 & .18593 & 5 & 3 & 2812 & .08225 & 3211 & .08911 \\
\hline 3271 & 2 & 38 & .25013 & 4 & 3 & 3241 & .11674 & 3272 & .02467 \\
\hline 3272 & 21 & 403 & .20655 & 6 & 1 & 3241 & .13780 & 3272 & .10749 \\
\hline 3273 & 18 & 576 & .35065 & 1 & 3 & 3241 & .02385 & 3272 & .07854 \\
\hline 3274 & 2 & 36 & .15427 & 7 & 3 & 2643 & .08248 & 3312 & .01182 \\
\hline 3281 & 4 & 47 & .15852 & 5 & 3 & 2643 & .04282 & 3949 & .04980 \\
\hline $\begin{array}{l}3291 \\
3292\end{array}$ & 3 & 46 & .03325 & 5 & 3 & 3291 & .01998 & 3291 & .01998 \\
\hline 3292 & 3 & 17 & .10211 & 5 & 2 & 2812 & .02575 & 3714 & .01433 \\
\hline 3293 & 4 & 77 & .11830 & 1 & 1 & 2499 & .02737 & 3714 & .02522 \\
\hline 3295 & 14 & 672 & .30024 & 4 & 8 & 3312 & .20741 & 2952 & .15879 \\
\hline $\begin{array}{l}3296 \\
3290\end{array}$ & 3 & 60 & .17934 & 4 & 5 & 2891 & .07565 & 3079 & .01079 \\
\hline 3299 & 3 & 124 & .40969 & 5 & 5 & 3299 & .24626 & 3299 & .24626 \\
\hline 3312 & 3 & 709 & .07970 & 2 & 2 & 3312 & .04791 & 3312 & .04791 \\
\hline 3313 & 2 & 216 & .58169 & 5 & 1 & 3334 & .56852 & 3312 & .23878 \\
\hline 3317 & 3 & 433 & .28146 & 2 & 2 & 3312 & .10426 & 3321 & .06932 \\
\hline 3321 & 12 & 4,046 & .28507 & 4 & 2 & 3312 & .17685 & 3312 & .17685 \\
\hline 3341 & 2 & 235 & .08446 & 2 & 4 & 2812 & .03649 & 3312 & .08136 \\
\hline 3356 & 2 & 6 & .13379 & 0 & 4 & - & - & 3861 & $.06963 \omega$ \\
\hline 3361 & 9 & 506 & .26747 & 2 & 1 & 3334 & .37926 & 3714 & .06856 \\
\hline
\end{tabular}


TABLE I (CON'T)

\begin{tabular}{|c|c|c|c|c|c|c|c|c|c|}
\hline \multirow{2}{*}{$\begin{array}{l}\text { SIC } \\
\text { CODE }\end{array}$} & \multirow[t]{2}{*}{ FIRMS } & \multirow[t]{2}{*}{ EMPLOYEES } & \multirow{2}{*}{$\begin{array}{l}\text { SELF } \\
\text { NEAREST } \\
\text { NEIGHBOR }\end{array}$} & \multicolumn{2}{|c|}{$\begin{array}{l}\text { NUMBER OF } \\
\text { LINKAGES }\end{array}$} & \multicolumn{2}{|c|}{$\begin{array}{l}\text { FIRST BACKWARD } \\
\text { LINKAGE }\end{array}$} & \multicolumn{2}{|c|}{$\begin{array}{l}\text { FIRST FORWARD } \\
\text { LINKAGE }\end{array}$} \\
\hline & & & & BACKWARD & FORWARD & SIC & $\mathrm{LC}$ & SIC & I.C \\
\hline 3362 & 8 & 171 & .18992 & 2 & 5 & 3565 & .05064 & 3494 & .02171 \\
\hline 3369 & 5 & 201 & .26687 & 2 & 2 & 3544 & .03258 & 3494 & .05015 \\
\hline 3398 & 3 & 48 & .18409 & 4 & 2 & 3312 & .16347 & 3312 & .16347 \\
\hline 3411 & 4 & 398 & .29837 & 3 & 4 & 3312 & .15365 & 2082 & .22217 \\
\hline 3412 & 4 & 148 & .16247 & 2 & 5 & 3312 & .08355 & 2992 & .03965 \\
\hline 3421 & 2 & 249 & .20080 & 7 & 3 & 3312 & .14774 & 2013 & .07820 \\
\hline 3423 & 5 & 488 & .22817 & 5 & 6 & 3312 & .17750 & 2431 & .08784 \\
\hline 3425 & 6 & 1,590 & .26887 & 3 & 3 & 3312 & .27110 & 2411 & .09749 \\
\hline 3429 & 11 & 1,088 & .16385 & 4 & 1 & 3312 & .17484 & 3711 & .18747 \\
\hline 3432 & 3 & 17 & .15436 & 4 & 5 & 3362 & .04642 & 2451 & .46825 \\
\hline 3433 & 12 & 897 & .25842 & 4 & 4 & 3312 & .23361 & 3433 & .07767 \\
\hline 3441 & 16 & 999 & .25720 & 2 & 7 & 3312 & .15625 & 3731 & .09920 \\
\hline 3442 & 28 & 835 & .19685 & 5 & 4 & 3354 & .15993 & 3792 & .06144 \\
\hline 3443 & 22 & 565 & .30356 & 2 & 5 & 3312 & .24441 & 3443 & .06738 \\
\hline 3444 & 53 & 1,017 & .22435 & 3 & 6 & 3312 & .19331 & 3792 & .08285 \\
\hline 3446 & 17 & 147 & .34393 & 5 & 2 & 3312 & .19313 & 3731 & .12926 \\
\hline 3448 & 3 & 59 & .16386 & 4 & 1 & 3312 & .09570 & 3448 & .09849 \\
\hline 3451 & 8 & 273 & .15130 & 3 & 3 & 3312 & .15523 & 3711 & .16624 \\
\hline 3462 & 3 & 139 & .13907 & 1 & 1 & 3312 & .05424 & 3714 & .05715 \\
\hline 3469 & 6 & 142 & .10841 & 2 & 2 & 3312 & .10960 & 3079 & .00754 \\
\hline 3471 & 17 & 604 & .10946 & 2 & 2 & 2899 & .06213 & 3677 & .02019 \\
\hline 3479 & 11 & 273 & .14341 & 6 & 2 & 2851 & .04519 & 3711 & .10768 \\
\hline 3484 & 4 & 44 & .46701 & 2 & 7 & 3484 & .24311 & 3312 & .22542 \\
\hline 3493 & 6 & 113 & .18646 & 4 & 1 & 3312 & .12676 & 3711 & .11171 \\
\hline 3494 & 14 & 795 & .19484 & 4 & 4 & 3312 & .15068 & 3585 & .06825 \\
\hline 3496 & 8 & 229 & .12588 & 2 & 4 & 3312 & .13892 & 2411 & .04100 \\
\hline 3499 & 5 & 25 & .22920 & 3 & 2 & 3312 & .15290 & 3312 & .15290 \\
\hline 3523 & 11 & 604 & .36099 & 4 & 1 & 3312 & .18457 & 3523 & .11332 \\
\hline 3524 & 2 & 17 & .29387 & 4 & 3 & 3312 & .15631 & 3524 & .21634 \\
\hline 3531 & 16 & 321 & .22022 & 5 & 2 & 3312 & .12246 & 3531 & .05732 \\
\hline
\end{tabular}


TABLE I (CON'T)

\begin{tabular}{|c|c|c|c|c|c|c|c|c|c|}
\hline \multirow[t]{2}{*}{$\begin{array}{l}\text { SIC } \\
\text { CODE }\end{array}$} & \multirow[t]{2}{*}{ F I RMS } & \multirow[t]{2}{*}{ EMPLOVEES } & \multirow{2}{*}{$\begin{array}{l}\text { SELF } \\
\text { NEAREST } \\
\text { NEIGHBOR }\end{array}$} & \multicolumn{2}{|c|}{$\begin{array}{l}\text { NUMBER OF } \\
\text { LINKAGES }\end{array}$} & \multicolumn{2}{|c|}{$\begin{array}{l}\text { FIRST BACKWARD } \\
\text { LINKAGE }\end{array}$} & \multicolumn{2}{|c|}{$\begin{array}{l}\text { FIRST FORWARD } \\
\text { LINKAGE }\end{array}$} \\
\hline & & & & BACKWARD & FORWARD & SIC & LC & SIC & LC \\
\hline 3532 & 3 & 625 & .25410 & 4 & 4 & 3312 & .17489 & 3732 & .15724 \\
\hline 3534 & 2 & 29 & .12787 & 3 & 4 & 3312 & .07391 & 3731 & .02363 \\
\hline 3535 & 9 & 314 & .18509 & 3 & 3 & 3312 & .18937 & 3531 & .06425 \\
\hline 3536 & 5 & 218 & .18490 & 4 & 4 & 3312 & .24245 & 3731 & .16060 \\
\hline 3537 & 15 & 1,907 & .20408 & 4 & 5 & 3312 & .16363 & 3537 & .05486 \\
\hline 3541 & 4 & 71 & .08638 & 6 & 5 & 3531 & .04496 & 3541 & .04496 \\
\hline 3544 & 19 & 173 & .21058 & 5 & 1 & 3312 & .19279 & 3544 & .05030 \\
\hline 3546 & 4 & 210 & .37340 & 4 & 4 & 3544 & .04980 & 2411 & .02843 \\
\hline 3551 & 4 & 32 & .13985 & 4 & 1 & 3312 & .11297 & 3551 & .07280 \\
\hline 3553 & 34 & 1,635 & .15307 & 4 & 7 & 3544 & .05911 & 2411 & .05571 \\
\hline 3554 & 5 & 187 & .23648 & 6 & 3 & 3554 & .11011 & 3554 & .11011 \\
\hline 3555 & $\begin{array}{l}2 \\
8\end{array}$ & 80 & .13100 & 5 & 5 & $355 ! j$ & .09644 & 3555 & \\
\hline 3559 & $\begin{array}{l}8 \\
6\end{array}$ & 342 & .18405 & 6 & 4 & 3312 & .17692 & 2812 & .08141 \\
\hline $\begin{array}{l}3561 \\
3564\end{array}$ & $\begin{array}{l}6 \\
6\end{array}$ & 1,097 & .06059 & 3 & 5 & 3324 & .02290 & $\begin{array}{l}3312 \\
3585\end{array}$ & \\
\hline $\begin{array}{l}3564 \\
3565\end{array}$ & $\begin{array}{r}6 \\
12\end{array}$ & $\begin{array}{l}822 \\
134\end{array}$ & $\begin{array}{r}.23610 \\
13850\end{array}$ & 2 & $\begin{array}{l}7 \\
2\end{array}$ & 3312 & $\begin{array}{r}.18774 \\
03716\end{array}$ & $\begin{array}{l}3585 \\
3324\end{array}$ & $\begin{array}{l}.06 / 83 \\
.04377\end{array}$ \\
\hline $\begin{array}{l}3565 \\
3566\end{array}$ & $\begin{array}{r}12 \\
3\end{array}$ & $\begin{array}{r}134 \\
33\end{array}$ & $\begin{array}{r}.13850 \\
.19008\end{array}$ & $\begin{array}{l}5 \\
5\end{array}$ & $\begin{array}{l}3 \\
3\end{array}$ & $\begin{array}{l}2421 \\
3312\end{array}$ & $\begin{array}{l}.03716 \\
.10637\end{array}$ & 3531 & .07497 \\
\hline 3567 & 5 & 136 & $\begin{array}{r}.19008 \\
.18674\end{array}$ & 3 & 4 & 3312 & .15889 & 3567 & .08695 \\
\hline 3569 & 5 & 94 & .20801 & 4 & 2 & 3312 & .13837 & 2812 & .06557 \\
\hline 3573 & 5 & 1,904 & .25576 & 3 & 1 & 3573 & .11908 & 3573 & .11908 \\
\hline 3576 & 2 & 14 & .13379 & 6 & 3 & 3079 & .03751 & 3576 & .09849 \\
\hline 3579 & 2 & 5 & .29387 & 4 & 2 & 3312 & .20778 & 3579 & .21634 \\
\hline 3581 & 2 & 18 & .25013 & 7 & 3 & 3312 & .14029 & 3581 & .18414 \\
\hline 3585 & 5 & 156 & .17850 & 3 & 2 & 3585 & .08311 & 3585 & .08311 \\
\hline 3589 & 7 & 190 & .25981 & 3 & 5 & 3312 & .23709 & 3589 & .10224 \\
\hline 3592 & 2 & 201 & .45372 & 6 & 3 & 3312 & .25266 & 3711 & .20983 \\
\hline 3599 & 103 & 1,821 & .18825 & 3 & 2 & 3599 & .01948 & 3599 & .01948 \\
\hline 3612 & 4 & 435 & .28908 & 2 & 2 & 3312 & .16328 & 3646 & .08620 \\
\hline 3613 & 8 & 412 & .26985 & 2 & 3 & 3622 & .07714 & 3743 & $.11327 \omega$ \\
\hline $2 a$ & 9 & 135 & .25579 & 5 & 4 & 3622 & .08877 & 3585 & $10831 \sigma$ \\
\hline
\end{tabular}


TABLE I (CON'T)

\begin{tabular}{|c|c|c|c|c|c|c|c|c|c|}
\hline \multirow{2}{*}{$\begin{array}{l}\text { SIC } \\
\text { CODE }\end{array}$} & \multirow[t]{2}{*}{ FIRMS } & \multirow[t]{2}{*}{ EMPLOYEES } & \multirow{2}{*}{$\begin{array}{l}\text { SELF } \\
\text { NEAREST } \\
\text { NEIGHBOR }\end{array}$} & \multicolumn{2}{|c|}{$\begin{array}{l}\text { NUMBER OF } \\
\text { LINKAGES }\end{array}$} & \multicolumn{2}{|c|}{$\begin{array}{l}\text { FIRST BACKWARD } \\
\text { LINKAGE }\end{array}$} & \multicolumn{2}{|c|}{$\begin{array}{l}\text { FIRST FORWARD } \\
\text { LINKAGE }\end{array}$} \\
\hline & & & & BACKWARD & FORWARD & SIC & $\mathrm{LC}$ & SIC & LC \\
\hline 3643 & 4 & 150 & .24375 & 3 & 6 & 3312 & .20773 & 3661 & .10385 \\
\hline 3645 & 7 & 79 & .13843 & 6 & 3 & 3612 & .06428 & 3711 & .10099 \\
\hline 3691 & 9 & 318 & .20643 & 3 & 2 & 3672 & .12898 & 3711 & .12993 \\
\hline 3661 & 3 & 465 & .32183 & 4 & 1 & 3661 & .19345 & 3661 & .19345 \\
\hline 3662 & 11 & 333 & .22953 & 3 & 3 & 3667 & .02591 & 3662 & .07205 \\
\hline 3674 & 4 & 2,812 & .17284 & 5 & 5 & 3677 & .03374 & 3662 & .04235 \\
\hline $\begin{array}{l}3677 \\
3691\end{array}$ & 16 & 1,184 & .23207 & 4 & 6 & 3677 & .06040 & 3662 & .06360 \\
\hline $\begin{array}{l}3691 \\
3693\end{array}$ & 6 & 379 & .25027 & 4 & 2 & 2819 & .10178 & 3711 & .19379 \\
\hline $\begin{array}{l}3693 \\
3694\end{array}$ & 6 & 203 & .18821 & 5 & 9 & 3672 & .14600 & 3693 & .07999 \\
\hline $\begin{array}{l}3694 \\
3713\end{array}$ & 7 & 176 & .23407 & 2 & 3 & 3694 & .09211 & 3711 & .16185 \\
\hline $\begin{array}{l}3713 \\
3714\end{array}$ & 7 & 170 & .30409 & 6 & 4 & 3711 & .11658 & 3711 & .11658 \\
\hline $\begin{array}{l}3714 \\
3715\end{array}$ & 33 & 972 & .19645 & 4 & 2 & 3312 & .17795 & 3711 & .18950 \\
\hline 3715 & 16 & 619 & .23355 & 4 & 2 & 3714 & .05183 & 3713 & .04836 \\
\hline 3728 & 4 & 1,634 & .46997 & 5 & 1 & 3728 & .24465 & 3728 & .24465 \\
\hline $\begin{array}{l}3731 \\
3732\end{array}$ & 13 & 2,493 & .09660 & 2 & 1 & 3312 & .09411 & 3731 & .02789 \\
\hline $\begin{array}{l}3732 \\
3743\end{array}$ & 17 & 84 & .23316 & 2 & 1 & 3211 & .10054 & 3732 & .05887 \\
\hline 3743 & 3 & 1,430 & .19359 & 3 & 2 & 3743 & .11636 & 3743 & .11636 \\
\hline 3751 & 5 & 127 & .24105 & 5 & 1 & 3731 & .11223 & 3751 & .11223 \\
\hline 3792 & 13 & 166 & .30879 & 5 & 1 & 3714 & .07152 & 3792 & .08916 \\
\hline 3799 & 4 & 60 & .08136 & 4 & 6 & 3312 & .21202 & 3799 & .04235 \\
\hline 3811 & 6 & 141 & .30897 & 4 & 3 & 3677 & .04893 & 3811 & .12561 \\
\hline 3822 & 2 & 284 & .02327 & 4 & 2 & 3079 & .03109 & 3585 & .11674 \\
\hline 3823 & 3 & 368 & .28744 & 2 & 3 & 3679 & .04785 & 3711 & .16061 \\
\hline 3825 & 6 & 15,562 & .15543 & 5 & 3 & 3679 & .04499 & 3825 & .06606 \\
\hline 3832 & 3 & 19 & .08445 & 5 & 2 & 3832 & .05076 & 3832 & .05076 \\
\hline 3841 & 10 & 609 & .12700 & 5 & 3 & 3079 & .01353 & 3842 & .21708 \\
\hline 3842 & 10 & 89 & .09459 & 6 & 1 & 2231 & .05699 & 3842 & .03114 \\
\hline 3843 & 8 & 148 & .23786 & 2 & 1 & 3356 & .18902 & 3843 & .08755 \\
\hline 3851 & 8 & 174 & .11797 & 5 & 3 & 3851 & .04342 & 3851 & .04342 \\
\hline 3861 & 6 & 900 & .10337 & 7 & 3 & 2812 & .07711 & 3861 & .04394 \\
\hline
\end{tabular}


TABLE I (CON'T)

\begin{tabular}{|c|c|c|c|c|c|c|c|c|c|}
\hline \multirow[t]{2}{*}{$\begin{array}{l}\text { SIC } \\
\text { CODE }\end{array}$} & \multirow[t]{2}{*}{ F IRMS } & \multirow[t]{2}{*}{ EMPLOYEES } & \multirow{2}{*}{$\begin{array}{l}\text { SELF } \\
\text { NEAREST } \\
\text { NEIGHBOR }\end{array}$} & \multicolumn{2}{|c|}{$\begin{array}{l}\text { NUMBER OF } \\
\text { LINKAGES }\end{array}$} & \multicolumn{2}{|c|}{$\begin{array}{l}\text { FIRST BACKWARD } \\
\text { LINKAGE }\end{array}$} & \multicolumn{2}{|c|}{$\begin{array}{l}\text { FIRST FORWARD } \\
\text { LINKAGE }\end{array}$} \\
\hline & & & & BACKWARD & FORWARD & SIC & IC & SIC & LC \\
\hline 3873 & 2 & 10 & .23849 & 1 & 3 & 3873 & .17557 & 3873 & .17557 \\
\hline 3911 & 10 & 47 & .11228 & 1 & 2 & 3356 & .07239 & 3911 & .03697 \\
\hline 3914 & 3 & 28 & .10449 & 7 & 3 & 3312 & .10135 & 3914 & .06281 \\
\hline 3931 & 4 & 228 & .32099 & 4 & 1 & 3931 & .16710 & 3931 & .16710 \\
\hline 3949 & 13 & 339 & .18271 & 4 & 1 & 2421 & .04170 & 3949 & .05276 \\
\hline 3953 & 8 & 81 & .05247 & 8 & 0 & 2499 & .03336 & - & - \\
\hline 3961 & 2 & 4 & .27642 & 2 & 2 & 3369 & .10286 & 3961 & .20349 \\
\hline 3964 & 3 & 23 & .32667 & 5 & 1 & 2231 & .11282 & 2321 & .04362 \\
\hline 3991 & 3 & 234 & .10221 & 6 & 7 & 3079 & .02929 & 3325 & .01719 \\
\hline 3993 & 16 & 180 & .13331 & 7 & 3 & 2821 & .09318 & 3993 & .12797 \\
\hline 3 & 3 & 21 & .06412 & 6 & 1 & 3995 & .03854 & 3995 & .03854 \\
\hline 3 & 8 & 134 & .21750 & 4 & 3 & 2651 & .05036 & 3999 & .08006 \\
\hline
\end{tabular}


adding the LC's of appropriate linked sectors. Again, limitations in the number of industries with more than 5 backward or forward linkages forced the exclusion of interindustry linkages $6,7,8$, and 9 from inclusion in the combinatorial analysis.

\section{AREA SELECTION}

Nations, regions, metropolitan areas, and local communities compete for the siting of new manufacturing facilities. Firms begin their search at the broadest geographical levels and eventually narrow their choices to local areas and sites. Trade-offs will be evaluated as each area and site will individually and in combination offer a different bundle of opportunities and constraints. But in the end it is the local regions and communities which furnish the sites on which the new facilities will be constructed. Therefore plant location theoretical formulations and empirical studies must include an examination of the siting process at the site, local, and regional levels.

Empirical studies have investigated the site selection process at the macro and micro levels of analysis. It is clearly recognized that it is inappropriate to generalize findings from the site to area level (Stanford Research Institute 1982). The findings at the macro level have been applied to the local area level by policy-makers 
and practitioners as the search goes on for propulsive or magnet industries which will stimulate development in the local economy, attract suppliers, and develop localized markets for goods and services (Oregon Economic Development Department 1977, Oregon Department of Land Conservation and Development 1973, Multnomah County 1977, and Batelle Memorial Institute 1967, 1975). The question is whether these policies and programs justified by empirical findings at the international, national and regional levels will work at the metrcpolitan scale. Recent findings by research/ practitioners have found that the attraction of associated industry to the community may not have the magnitude of impact expected (Sommers 1984). This study focused on the relevancy of hypotheses generated in the literature through micro level theory development and empirical . studies done at various levels, with the exception of the micro one.

Most manufacturing firms prefer a site within a metropolitan area (U.S. Department of Commerce 1973). Sites selected present a picture of the end result of the locational search process. The metropolitan area chosen for study must have the following attributes if the influences of intra-industry and inter-industry linkages are to be assessed.

1. Subarea differences on influential variables 
other than the linkage variables should be minimal.

2. Multi-modal transshipment nodes should be available and transportation infrastructure offer a choice of transportation modes at competitive costs

3. Utilities should be available and costs of delivered services competitive

4. An inventory of buildable sites must have been available across time.

5. A diversified manufacturing base with a relatively large number of firms capable of supporting intra-industry and inter-industry networks

6. A stable community framework which supports economic growth and diversification

The Oregon portion of the Portland-Vancouver SMSA meets these requirements. Subarea cost variations for land, labor, and capital are minimal although the usual differences between the central city and suburban fringe do exist. These differences do not, however, distinguish the Portland metropolitan area from other medium sized metropolitan areas. A full range of industrial sites with varying locational features and levels of public infrastructure have been historically available within the urban and urbanizable portions of the area (Baldwin, 1982) although a recent study (Stanford Research Institute 1982) questions the validity of this assertion in the 1980's for 
the attraction of a full range of industry.

The Portland-Vancouver SMSA was credited with having the eleventh most diversified manufacturing base of all metropolitan areas in 1975 and ranked eighth in volume of tonnage shipped and received in 1977 (Multnomah County 1977). The Counties of Multnomah, Clackamas, and Washington which make-up the Oregon portion of the SMSA contains identified 2204 digit Standard Industrial Classification manufacturing industries representing an estimated 2,111 firms with an estimated combined labor force of 108,295 in 1977 .

The Portland-Vancouver SMSA serves as the State's commercial and distribution center. Its strategic location on the Columbia River fostered its development as a transshipment point for goods to and from the inland areas. Utility costs, particularly for electricity, are comparatively low (Stanford Research Institute 1982). Capital for development has been historically available although until recently natural resource oriented industries were favored by local investors over high technology economic activities (First Interstate Bank, 1977). Historically, public land use policies and regulations have been flexible, allowing industry to locate where site requirements could be met with minimal publicly imposed constraints (Baldwin 1982). Until recently the availability of federal grants to support public infrastructure development allowed local communities to keep 
pace with industries' demand for physical and social infrastructure. Differences in political policy, taxation, transportation freight rates, and utility costs required the deletion of clark County Washington, the fourth County of the metropolitan area from this study.

The time chosen for the study was 1977. Major changes in public policies and regulations came about with the adoption of the Statewide Land Use Planning Goals and Urban Growth Boundary Strategies in 1973 and the first of the major jurisdictional comprehensive land use in compliance with new statewide requirements. The impact of these regulations and resulting land use plans are a matter of some controversy. A study done by 1000 Friends of oregon (1982) concludes that the inventory of buildable and industrially zoned sites increased substantially as a result of these plans. As has already been discussed the Stanford Research Institute (1982) questions the adequacy of current industrial site inventories. In addition, the current economic recession which took hold on the metropolitan area economy in 1979 and federal policy shanges in the 1980's have created substantial shifts in the marketplace and availability of infrastructure capital. The post 1977 period represents a time of changing conditions for the regional, state, and metropolitan area economies, Local governments have adopted comprehensive land use plans and implementing ordinances under the 
Statewide Planning Goals and Guidelines. Demand for Oregon wood products has declined affecting the employment bases of many Oregon communities as more than 40 percent of the State's manufacturing employment is dependent upon the lumber and wood products industries (Multnomah County 1977). The majority of the growth in the high technology instruments industrial grouping has occurred in the late $1970^{\prime}$ 's and 1980's. Federal funding for new infrastructure development is being severely curtailed at a time when oregon communities are now under a 1983 legislative directive to develop long range public facilities and services plans (Oregon Legislature 1983). The national recession which hit Oregon in 1979 and still cripples much of the State's economy has led to increased efforts to develop a statewide approach to economic development (Oregon Legislature 1983). How these shifts in the marketplace, resource availability, new political approaches, and land use regulations will affect future opportunities for economic growth and diversification in the long run is not yet known. This dissertation in its investigation of the influence of intra- and inter-industry linkages on firm spatial proximity at the metropolitan level, depending on the results obtained, will suggest the continued use or reassessment of a strategy applied locally in an attempt to overcome existing economic conditions. 


\section{VARIABLE MEASUREMENT}

Spatial Proximity

The intra-industry spatial proximity of firms is measured with the use of the Nearest Neighbor coefficients and inter-industry firm spatial proximity by the use of Local Concentration coefficients. The areal dimensions for both equations take into account the actual area in which manufacturing firms could site by the subtraction of subareas within the 3 Counties within federal and state national forests and recreation areas such as the Mt. Hood National Forest. The inclusion of natural resource and urban oriented manufacturing industries precluded the need for further modification of area measurements to differentiate between urban and rural areas.

Firms were mapped by industry and by plant address on milar overlays over a $1 "=2000 \mathrm{ft}$. base scale map of the Portland metropolitan area. A list of firms and employment by industry is displayed in Table I. Inter-firm distance measures were made and rounded to the nearest tenth of an inch and then converted into miles and feet. A reliability check on a randomly selected 10 percent sample of measures indicated that over 99 percent of the measurements were accurate within one-tenth of an inch on the base map. Table II lists the study variables and gives definitions for each one used. 
TABLE II

LIST OF VARIABLES

Dependent Variables

Spatial Proximity of Intra-industry Firms

Spatial Proximity of Inter-industry Firms

Covariates

Industry Average Firm Size

Manufacturing Purchases as a Percentage of Total

Purchases in the Industry

Manufacturing Sales as a Percentage of Total

Industry Sales

Transportation Purchases as a Percentage of Total Industry Purchases

Utility Purchases as a Percentage of Total Industry Purchases

Experimental Variables

Percentage of Intra-industry Purchases Among All Firms in Industry I to Total Manufacturing Purchases

Percentage of First Backward Linkage Purchases to Total Manufacturing Purchases

Percentage of Second Backward Linkage Purchases to Total Manufacturing Purchases

Percentage of Third Backward Linkage Purchases to Total Manufacturing Purchases

Percentage of Fourth Backward Linkage Purchases to Total Manufacturing Purchases

Percentage of Fifth Backward Linkage Purchases to Total Manufacturing Purchases

Percentage of First Forward Linkage Sales to Total Manufacturing Sales

Percentage of Second Forward Linkage Sales to Total Manufacturing Sales

Percentage of Third Forward Linkage Sales to Total Manufacturing Sales

Percentage of Fourth Forward Linkage Sales to Total Manufacturing Sales

Percentage of Fifth Forward Linkage Sales to Total Manufacturing Sales 
Linkages

To identify within and between industry trade flows theorists and researchers have traditionally used location quotients and input-output transactions tables. These tables identify inter-industry transactional flows (Leontief 1951), analyze industry interlocking dependencies and derive estimates how changes in one industry will impact other industries and sectors of the economy (Nourse 1968; Richardson 1972; Hewings 1977).

The use of input-output transactional tables at the 4 digit SIC level of disaggregation is most appropriate for this study. The problems of the use of: more aggregated data are already noted and the use of a jiner screen requires the grouping of firms into too great a number of categories for use at the metropolitan level of analysis. Input-output transactional tables describe the magnitude of inter-industry industry exchanges of goods and services in dollars--the information needed to develop intra-industry and interindustry linkages. Level of product export is not relevant for this study as the focus is upon within area intraindustry and inter-industry linkage networks and participants. Industries were excluded from the firm set if no production facilities were present locally. Headquarters facilities were also excluded although any local production facilites were included in the industry set. Inter-industry technological coefficients are used to 
describe the magnitude of product and service exchanges among established inter-industry trading partners. Stability of measurement rather than fluctuations in relationships sensitive to short-term changing market conditions is the issue. Stability is an assumption on which input-output models is based although walker (1982) notes that adjustments may be needed to apply national or regional coefficients at the local level. Tiebout (1962) suggested a questionnaire method which could be used to make these adjustments and apply this analysis technique at the local level. Ettlinger (1984) criticizes conventional Kenseyian multiplier base linkages because of the organizational factors of firms which inhibit local linkages. Gibson and Worden (1981) suggest that the minimum requirements approach gives the best estimate of census survey multipliers. Tiebout (1956) and Isard (1960) recognizing the greater stability of the national coefficients over time caution researchers using a longitudinal time frame in the research design about the compounding of measurement problems likely with the use of regional rather than national measures. This study although using a 1977 cross-sectional data bank about plant location is using input-output locational coefficients to describe established interindustry linkages most likely to lead to cost-reductions or revenue generation and be considered as a location factor by firms. These inter-industry measures are treated as 
technological Iinkages rather than reflectors of actual transactional flow patterns within the Portland regional economy.

The Detailed Input-Output Structure of the U.S Economy 1972 (U.S. Department of Commerce, Bureau of Economic Analysis 1979) Use of Commodities by Industry table was used to obtain intra-industry and inter-industry records of purchases and sales. Firms are grouped into commodity/industrial classes equivalent in almost all cases to a 4 digit SIC category. Table I lists the number of industries, firms, and employment in the metropolitan area within each commodity/industry class and SIC categorical equivalences.

Industries with no plant in the region were dropped from the analysis and plants missing from the original data set were added. Table XIX in the Appendix lists by industry and employment 223 firms added to the original firm 1ist. Total firm deletions were 106: 44 were out of area; 41 could not be located, and 21 firms were actually headquarters facilities or closed.

Additions resulted in a regional manufacturing employment estimate of 108,295 with a net increase of 3,640 due to firm list refinement. Sixteen industries with only one facility in the region were used in the analysis as a supplier of inputs or purchaser of outputs but could not be treated directly as a subject firm due to measurement 
requirements of the Nearest Neighbor and Local Concentration formulations. The data base was composed of 220 industries with 2,111 firms.

Norcliffe (1975) found that the use of a modified location quotient produced scores which were roughly equivalent in their magnitude to input-out coefficients with a data base organized at the 4 digit Standard Industrial Classification level. In a similar study Isserman (1977) found that much of the unreliability of location quotients in previous research was associated, again, with the heterogeneity of industrial categories used. Input-output coefficients were used in this study at the 4 digit SIC level.

Transportation and Utility Costs

Expenditures on transportation and related services and utilities were obtained from the input-output transactions table (U. S. Department of Commerce, Bureau of Economic Analysis 1979). Total transportation costs include expenditures for passenger miles and commodity movement by highway, truck, rail, air, water, and pipeline and related transportation services. Utilities include expenditures for water, sanitary sewer, gas, and electricity. For each the subcategories were summed and defined in terms of the percentage of total industry purchases they represented. 
Supply and Market Orientation

Manufacturing supply (material) orientation is the percent of total expenditures made spent for manufacturing inputs. Market orientation is the percent of total sales made to manufacturing firms. Industries vary in the proportion of their purchases and sales that are made with other manufacturing plants.

The location of other manufacturing facilities would not be expected to be important for other firms who purchase or sell a majority of their inputs and outputs outside the manufacturing sector of the economy. As with transportation and utility costs these variables are treated as covariates within the model.

Industry Average Firm Size

The prepondence of the studies in location theory which have included firm size as a variable have found effects attributable to firm size. Industry average firm size equals the total number of employers in the industry divided by the number of firms. The purpose of this variable's inclusion as a covariate is to account for the variability in the dependent variable, spatial proximity, attributable to firm size.

The smallest firm in the firm subset had 1 employee and the largest just over 9,000. The smallest subject industry had 4 employees and the largest 15,562. The largest 
number of firms per industry was 171 (Table I).

\section{CONCLUSION}

To summarize, it is hypothesized that intra-industry and primary inter-industry linkages are not significant determinants of firm location within a metropolitan economy. It is also expected that spatial proximity of firms is not related to the magnitude of their composite forward and backward linkages. Failure to reject the null hypotheses will provide support for the research hypotheses as stated earlier in this chapter. 
CHAPTER IV

FINDINGS

Multiple regression covariance applications were used to examine the relationships of intra-industry and inter-industry linkages to firm spatial proximity. Variation in the dependent variable attributable to interindustry linkages was also examined for combinations of backward and forward linkages.

The treatment of average firm size, supply and market orientations, and transportation and utility industry intensities as covariates allowed their aggregate influence on the dependent variable to be controlled prior to measurement of the impacts of the experimental variables. The utility cost index is composed of water, electric, and sanitary sewer services purchases. The transportation cost composite index covers expenditures for passenger travel and movement of commodities by truck, water, air, rail, and pipleline. Colinearity between the covariates and the experimental variables was not an issue in this study. Considering all regression runs, association among covariates and experimental variables ranged from a low of -.2606 between the first forward linkage and market orientation in the first forward linkage model to .37109 between the second backward linkage and transportation 
purchases in the three backward and three forward linkage model.

To reiterate, the research hypotheses of this study are:

1. The spatial proximity of firms in an industry is not related to the magnitude of intra-industry technological linkages.

2. The spatial proximity of firms is not related to the magnitude of their primary forward and backward linkages.

3. The spatial proximity of firms is not related to magnitude of their composite forward and backward linkages.

SUMMARY OF REGRESSION FINDINGS

Applications were considered for intra-industry linkages (Table III and IV), 5 backward linkages (Tables VVII), 2 backward combinations (Table VIII), 5 forward linkages (Tables IX-XI), 2 forward combinations (Table XII), and 9 combinations of up to 3 forward and backward linkages (Tables XIII-XVII). Attrition of industries when using inter-industry linkages in combination precluded the use of 4 th and 5 th linkages together with the other inter-industry linkages.

Two multiple regression models had a overall $\mathrm{F}$ ratio significant at the .05 level: 1) intra-industry linkages when the covariate market orientation rather than supply 


\section{REGRESSION OF INTRA-INDUSTRY LINKAGE WITH COVARIATES INCLUDING MANUFACTURING PURCHASES}

\begin{tabular}{|c|c|c|c|}
\hline VARIABLE & $\begin{array}{l}\text { REGRESSION } \\
\text { COEFF ICIENT }\end{array}$ & $\begin{array}{l}\text { STANDARD } \\
\text { ERROR B }\end{array}$ & $\begin{array}{l}\text { F, REGRESSION } \\
\text { COEFFICIENT }\end{array}$ \\
\hline $\begin{array}{l}\text { Utility } \\
\text { Purchases }\end{array}$ & 1.3495 & 0.5369 & $6.319 * * *$ \\
\hline $\begin{array}{l}\text { Manufacturing } \\
\text { Purchases }\end{array}$ & 0.0203 & 0.0671 & 0.092 \\
\hline $\begin{array}{l}\text { Average Firm } \\
\text { Size }\end{array}$ & -0.0011 & 0.0041 & 0.071 \\
\hline $\begin{array}{l}\text { Transportation } \\
\text { Purchases }\end{array}$ & 0.0439 & 0.3861 & 0.013 \\
\hline $\begin{array}{l}\text { Intra-Industry } \\
\text { Transactions }\end{array}$ & 0.0296 & 0.0539 & 0.301 \\
\hline Constant & 17.9119 & & \\
\hline $\begin{array}{l}\mathrm{R}^{2}=0.0392 \quad \text { ADJUSTED } \\
\text { STANDARD ERROR }=11.5597\end{array}$ & $\mathrm{R}^{2}=0.0168$ & $\mathrm{~F}=1.7481$ & \\
\hline
\end{tabular}


TABLE IV

REGRESSION OF INTRA-INDUSTRY LINKAGE WITH COVARIATES INCLUDING MANUFACTURING

SALES

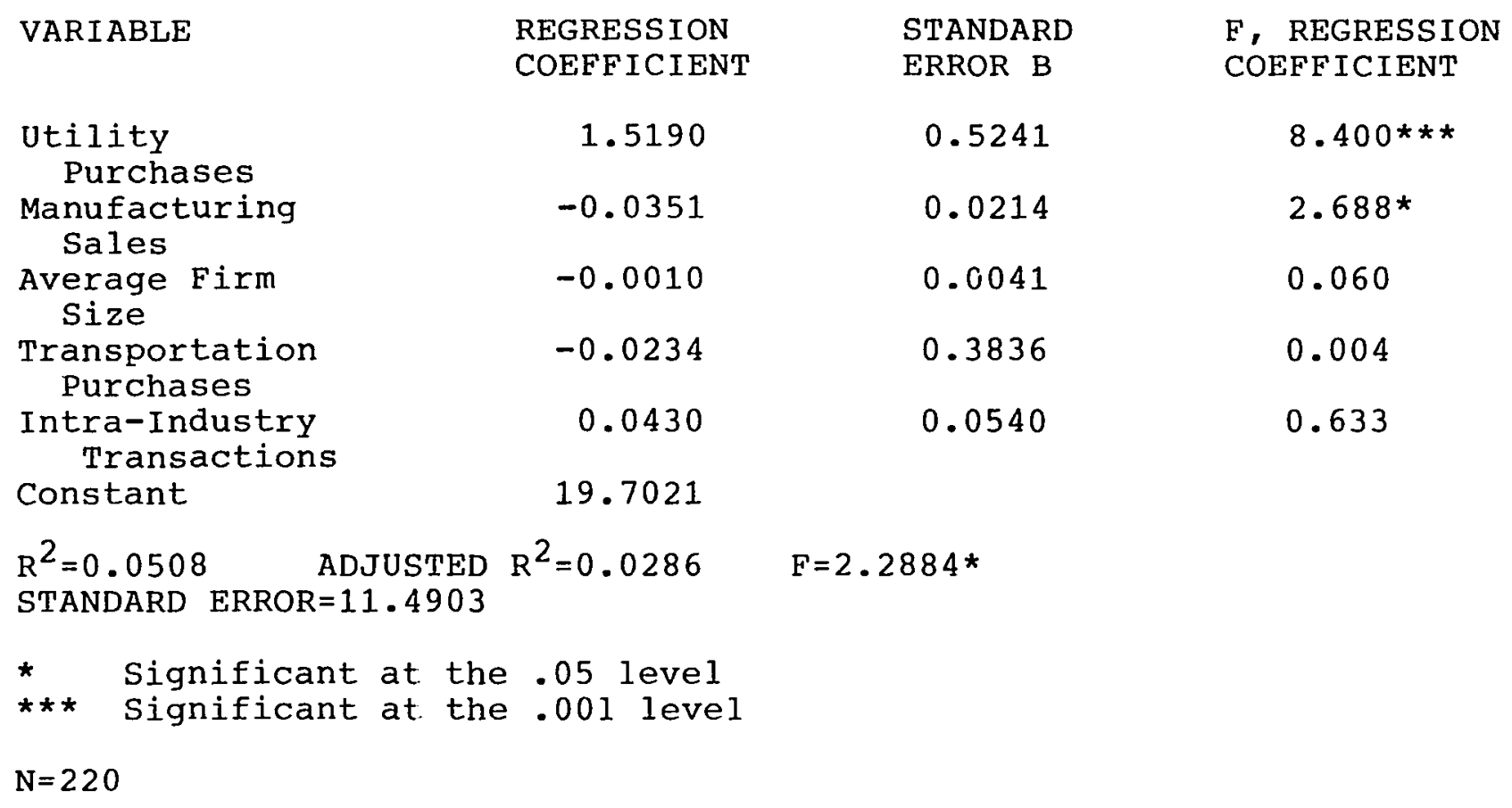


REGRESSION OF FIRST BACKWARD LINKAGE WITH COVARIATES

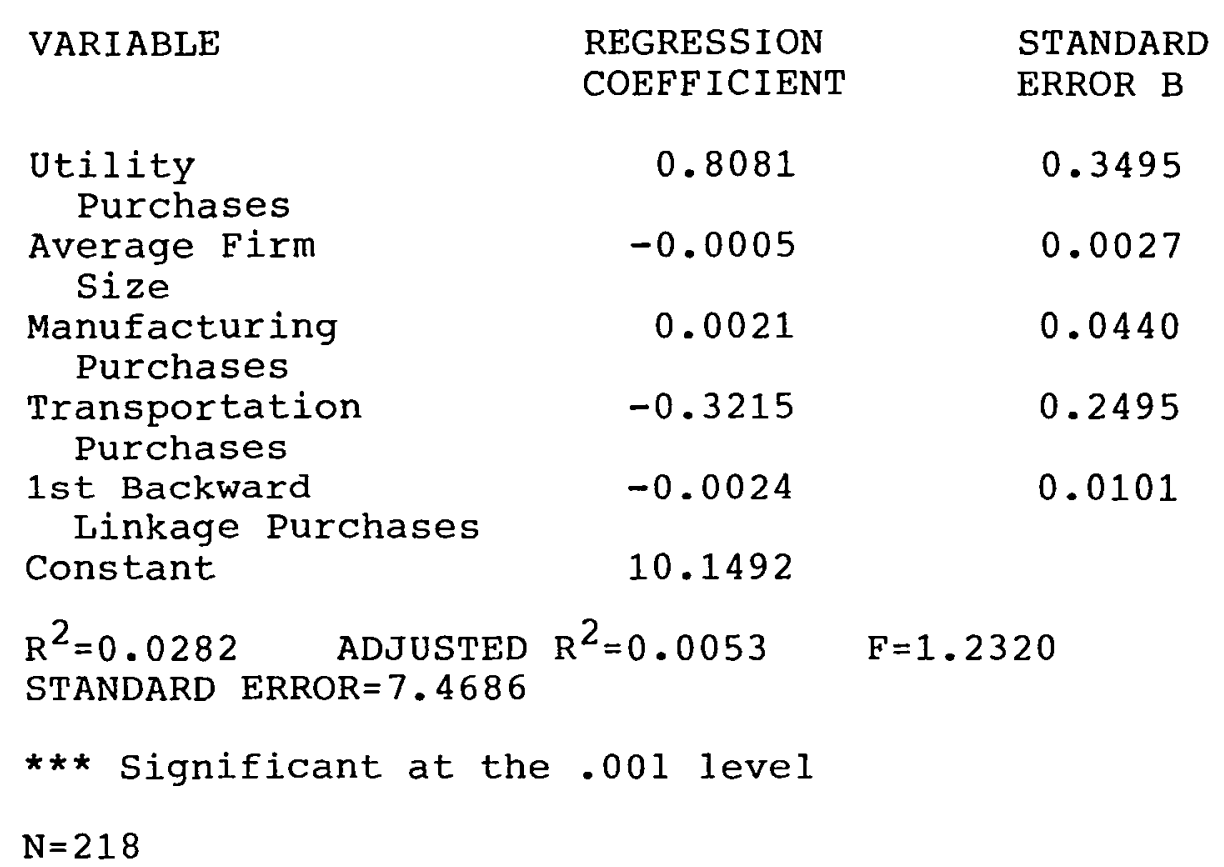

\section{F, REGRESSION COEFFICIENT}

$5.345 * \star *$

0.031

0.002

1.660

0.058 
TABLE VI

REGRESSIONS OF SECOND BACKWARD AND THIRD BACKWARD LINKAGES WITH COVARIATES

\section{VARIABLE}

utility

Purchases

Average Firm

$$
\text { Size }
$$

Manufacturing Purchases

Transportation

Purchases

2nd Backward

Linkage Purchases

3rd Backward

Linkage Purchases Constant

$$
\mathrm{R}^{2}
$$

ADJUSTED $\mathrm{R}^{2}$

F-RATIO

STANDARD ERROR

* Significant at the .05 level

$N=206$
$\mathrm{Y}=2 \mathrm{ND}$ BACKWARD LINKAGE LC

$X=1,2,3,4,8$

REGRESSION STANDARD $F$, REGRESSION COEFFICIENT ERROR B COEFFICIENT

$\begin{array}{rrr}0.2133 & 0.3602 & 0.350 \\ -0.0002 & 0.0026 & 0.006 \\ 0.0312 & 0.0447 & 0.488 \\ -0.0470 & 0.2728 & 0.030 \\ -0.0237 & 0.0639 & 0.138\end{array}$

8.6195

0.0048

$-0.0200$

0.1946

7.2414
$Y=3 R D$ BACKWARD LINKAGE LC $\mathrm{X}=1,2,3,4,9$ REGRESSION STANDARD F, REGRESSION COEFFICIENT ERROR B COEFFICIENT

$\begin{array}{rrr}0.5395 & 0.3809 & 2.006 \\ 0.0003 & 0.0026 & 0.014 \\ -0.0914 & 0.0539 & 2.874 * \\ 0.0398 & 0.3005 & 0.018 \\ 0.0886 & 0.1888 & 0.220 \\ 10.4066 & & \end{array}$

$$
\begin{aligned}
& 0.0613 \\
& 0.0316 \\
& 2.0620
\end{aligned}
$$$$
10.3014
$$

$\mathrm{N}=164$ 
REGRESSIONS OF FOURTH BACKWARD AND FIFTH BACKWARD LINKAGES WITH COVARIATES

VARI ABLE

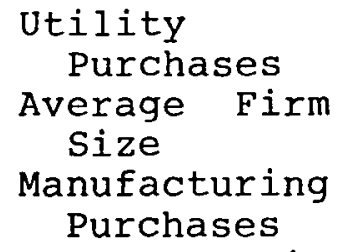

$\mathrm{N}=116$
$Y=4 T H \quad B A C K W A R D$ LINKAGE LC $\mathrm{X}=1,2,3,4,10$

REGRESSION STANDARD $F$, REGRESSION COEFFICIENT ERROR B COEFFICIENT

$\begin{array}{rll}0.8127 & 0.3236 & 6.306 * * \star \\ -0.0002 & 0.0021 & 0.009 \\ -0.0797 & 0.0538 & 2.193 \\ -0.2926 & 0.2469 & 1.405 \\ 0.1596 & 0.2556 & 0.390\end{array}$

5.3934

0.0600

0.0173

1.4052

5.4697
$\mathrm{Y}=5 \mathrm{TH}$ BACKWARD LINKAGE LC $\mathrm{X}=1,2,3,4,11$

REGRESSION STANDARD F, REGRESSION COEFFICIENT ERROR B COEFFICIENT

$\begin{array}{lll}0.5820 & 0.4529 & 1.651 \\ 0.0058 & 0.0023 & 6.430 * * * \\ 0.0727 & 0.0805 & 0.816 \\ 0.2375 & 0.3746 & 0.402\end{array}$

$$
\begin{array}{lll}
-1.0579 & 0.6027 & 3.080 *
\end{array}
$$

10.5019

0.2091

0.1359

2.8550 *

5.8843

$\mathrm{N}=60$ 
REGRESSIONS OF FIRST AND SECOND BACKWARD AND

FIRST, SECOND, AND THIRD BACKWARD

LINKAGES WITH COVARIATES

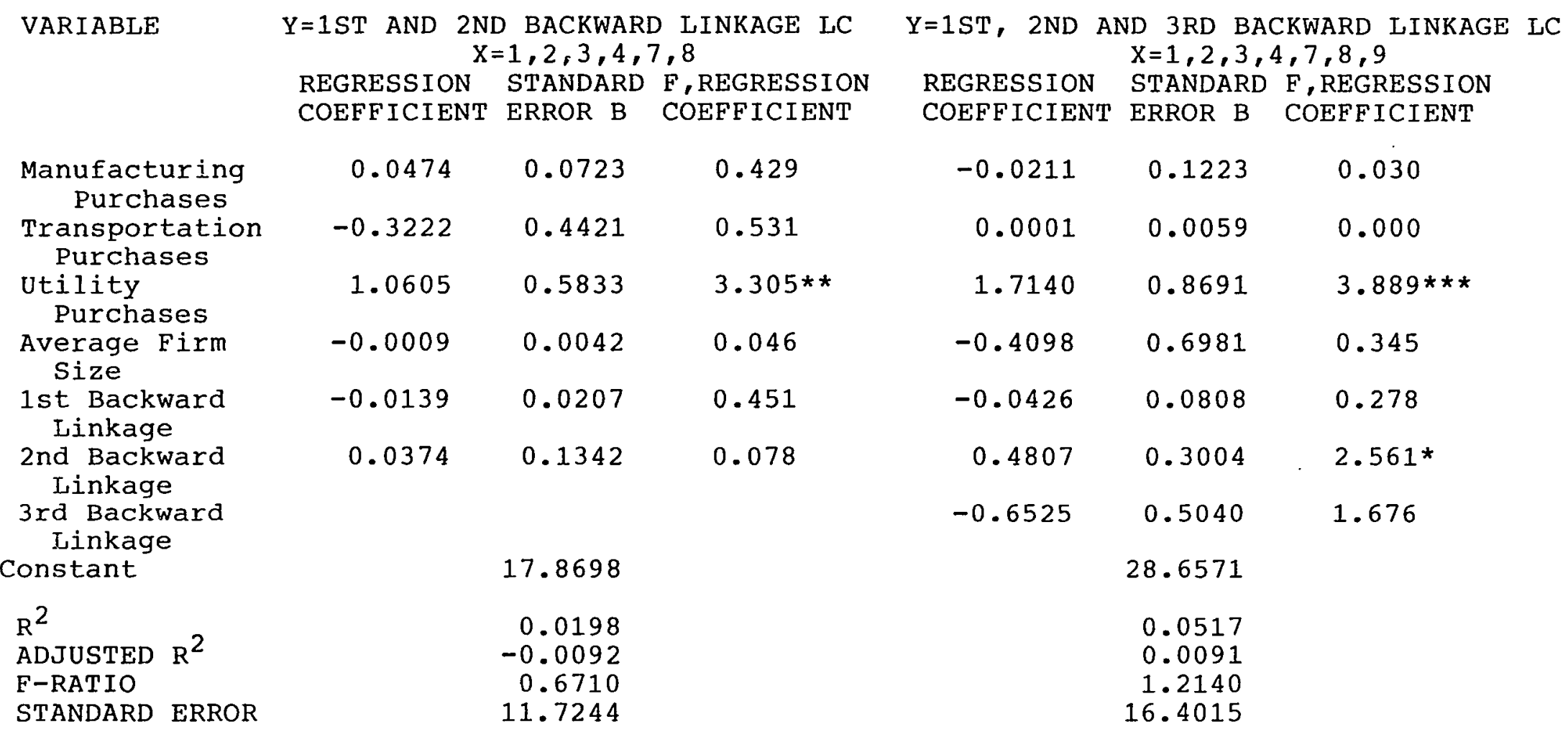

* Significant at the .05 level

** Significant at the .01 level

$\star * *$ Significant at the .001 level

$\mathrm{N}=206$ 
TABLE IX

REGRESSION OF FIRST FORWARD LINKAGE WITH COVARIATES

\begin{tabular}{|c|c|c|c|}
\hline VARIABLE & $\begin{array}{l}\text { REGRESSION } \\
\text { COEFF ICIENT }\end{array}$ & $\begin{array}{l}\text { STANDARD } \\
\text { ERROR B }\end{array}$ & $\begin{array}{l}\text { F, REGRESSION } \\
\text { COEFFICIENT }\end{array}$ \\
\hline $\begin{array}{l}\text { Average Firm } \\
\text { Size }\end{array}$ & 0.0002 & 0.0024 & 0.004 \\
\hline $\begin{array}{l}\text { Manufacturing } \\
\text { Sales }\end{array}$ & -0.0124 & 0.0127 & 0.965 \\
\hline $\begin{array}{l}\text { Transportation } \\
\text { Purchases }\end{array}$ & -0.0878 & 0.2277 & 0.149 \\
\hline $\begin{array}{l}\text { Utility } \\
\text { Purchases }\end{array}$ & 0.3665 & 0.3350 & 1.197 \\
\hline $\begin{array}{l}\text { 1st Forward } \\
\text { Linkage Sales }\end{array}$ & -0.0001 & 0.0003 & 0.127 \\
\hline Constant & 9.2594 & & \\
\hline $\begin{array}{l}\mathrm{R}^{2}=0.0082 \quad \text { ADJUSTE:D } \\
\text { STANDARD ERROR }=6.7763\end{array}$ & $\mathrm{R}^{2}=-0.0153$ & $\mathrm{~F}=0.3499$ & \\
\hline
\end{tabular}


TABLE X

REGRES:IIONS OF SECOND FORWARD AND THIRD FORWARD LINKAGES WITH COVARIATES

\section{VARIABLE}

Average Firm Size

Manufacturing

$$
\text { Sales }
$$

Transportation

Purchases

Utility

Purchases

2nd Forward

Linkage Sales

3rd Forward

Linkage Sales

Constant.

$$
\mathrm{R}^{2}
$$

ADJUSTED $\mathrm{R}^{2}$

F-RATIO

STANDARD ERROR

* Significant at the .05 level
$\mathrm{Y}=\mathrm{SECOND}$ FORWARD LINKAGE LC

$\mathrm{X}=1,2,3,4,13$

REGRESSION STANDARD F, REGRESSION COEFFICIENT ERROR B COEFFICIENT

$\begin{array}{rll}-0.0021 & 0.0024 & 0.755 \\ -0.0062 & 0.0 .43 & 0.186 \\ 0.2711 & 0.2495 & 1.181 \\ -0.7014 & 0.4387 & 2.556 * \\ 0.0358 & 0.0372 & 0.930\end{array}$

8.991

0.0357

0.0061

1.2074

6.6483

\section{$Y=T H I R D$ FORWARD LINKAGE LC} $X=1,2,3,4,14$

REGRESSION STANDARD F, REGRESSION

COEFFICIENT ERROR B COEFFICIENT

$\begin{array}{lll}-0.0013 & 0.0035 & 0.134\end{array}$

$\begin{array}{lll}0.0269 & 0.0218 & 1.529\end{array}$

$\begin{array}{lll}0.4501 & 0.3937 & 1.307\end{array}$

$\begin{array}{lll}-0.5281 & 0.6680 & 0.625\end{array}$

$\begin{array}{lll}-0.2520 & 0.2046 & 1.517\end{array}$

11.1275

0.0383

$-0.0067$

0.8513

8.9967

$N=169$ 
TABLE XI

REGRESSIONS OF FOURTH FORWARD AND FIFTH FORWARD LINKAGES WITH COVARIATES

\section{VARIABLE}

$$
\begin{aligned}
& \text { Average Firm } \\
& \text { Size }
\end{aligned}
$$

Manufacturing Sales

Transportation

Purchases

Utility

Purchases

4 th Forward

Linkage Sales

5 th Forward

Linkage Sales

Constant

$\mathrm{R}^{2}$

ADJUSTED $\mathrm{R}^{2}$

F-RATIO

STANDARD ERROR

$\mathrm{N}=65$
$Y=F O U R T H \quad$ FORWARD LINKAGE LC

$$
\mathrm{X}=1,2,3,4,15
$$

REGRESSION STANDARD F, REGRESSION COEFFICIENT ERROR B COEFFICIENT

$\begin{array}{rr}-0.0020 & 0.0089 \\ 0.0316 & 0.0274 \\ 0.1783 & 0.4933 \\ 0.4633 & 1.0169 \\ 0.5827 & 0.4063 \\ & \\ & 2.7211 \\ & 0.0747 \\ & -0.0037 \\ & 0.9532 \\ & 6.6971\end{array}$

$Y=F I F T H \quad$ FORWARD LINKAGE LC $\mathrm{X}=1,2,3,4,16$

REGRESSION STANDARD F, REGRESSION COEFFICIENT ERROR B COEFFICIENT

$\begin{array}{rrr}0.0012 & 0.0080 & 0.024 \\ 0.0189 & 0.0367 & 0.266 \\ 0.5001 & 0.5094 & 0.964 \\ -1.2752 & 1.1822 & 1.164 \\ & & \\ 0.2789 & 0.5981 & 0.217 \\ & 6.9580 & \\ & 0.0611 & \\ & -0.1065 & \\ & 0.3646 & \\ & 5.4893 & \end{array}$

$\mathrm{N}=34$ 
TABLE XI I

REGRESSIONS OF FIRST AND SECOND FORWARD AND
FIRST, SECOND, AND THIRD FORWARD

LINKAGES WITH COVARIATES

\begin{abstract}
VARIABLE
$\mathrm{Y}=1 \mathrm{ST}$ AND 2ND FORWARD LINKAGE LC $\mathrm{X}=1,2,3,4,12,13$

REGRESSION STANDARD F, REGRESSION COEFFICIENT ERROR B COEFFICIENT
\end{abstract}

$\begin{array}{rll}-0.9272 & 0.6630 & 1.956 \\ -0.0031 & 0.0037 & 0.698 \\ -0.0062 & 0.0216 & 0.082 \\ 0.0619 & 0.3765 & 0.027 \\ 0.0002 & 0.0004 & 0.149 \\ 0.0242 & 0.0631 & 0.147\end{array}$

$Y=1 S T$, 2ND AND 3RD FORWARD LINKAGE IC $X=1,2,3,4,12,13,14$ REGRESSION STANDARD F, REGRESSION COEFFICIENT ERROR B COEFFICIENT

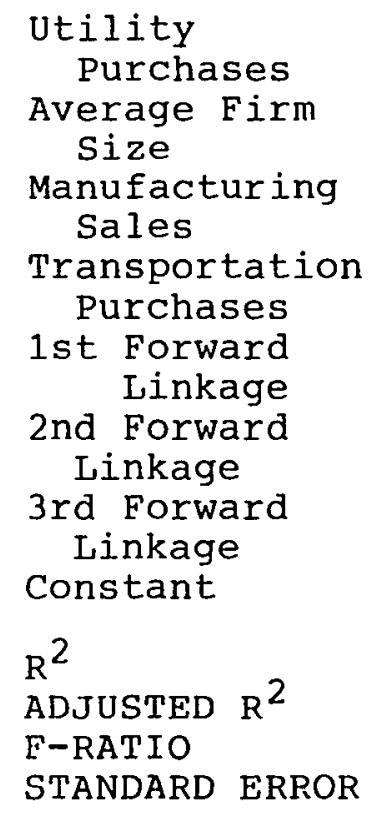

$\begin{array}{rrr}-1.7375 & 1.1457 & 2.300 * \\ -0.0052 & 0.0059 & 0.785 \\ 0.0352 & 0.0378 & 0.863 \\ 0.8133 & 0.6745 & 1.454 \\ 0.0445 & 0.0823 & 0.292 \\ -0.0785 & 0.1523 & 0.266 \\ -0.0273 & 0.3774 & 0.005 \\ & 27.2431 & \\ & 0.0381 & \\ & -0.0260 & \\ 0.5940 & \\ 15.4057 & \end{array}$


REGRESSIONS OF ONE BACKWARD AND ONE FORWARD LINKAGES AND ONE BACKWARD AND TWO FORWARD LINKAGES WITH COVARIATES

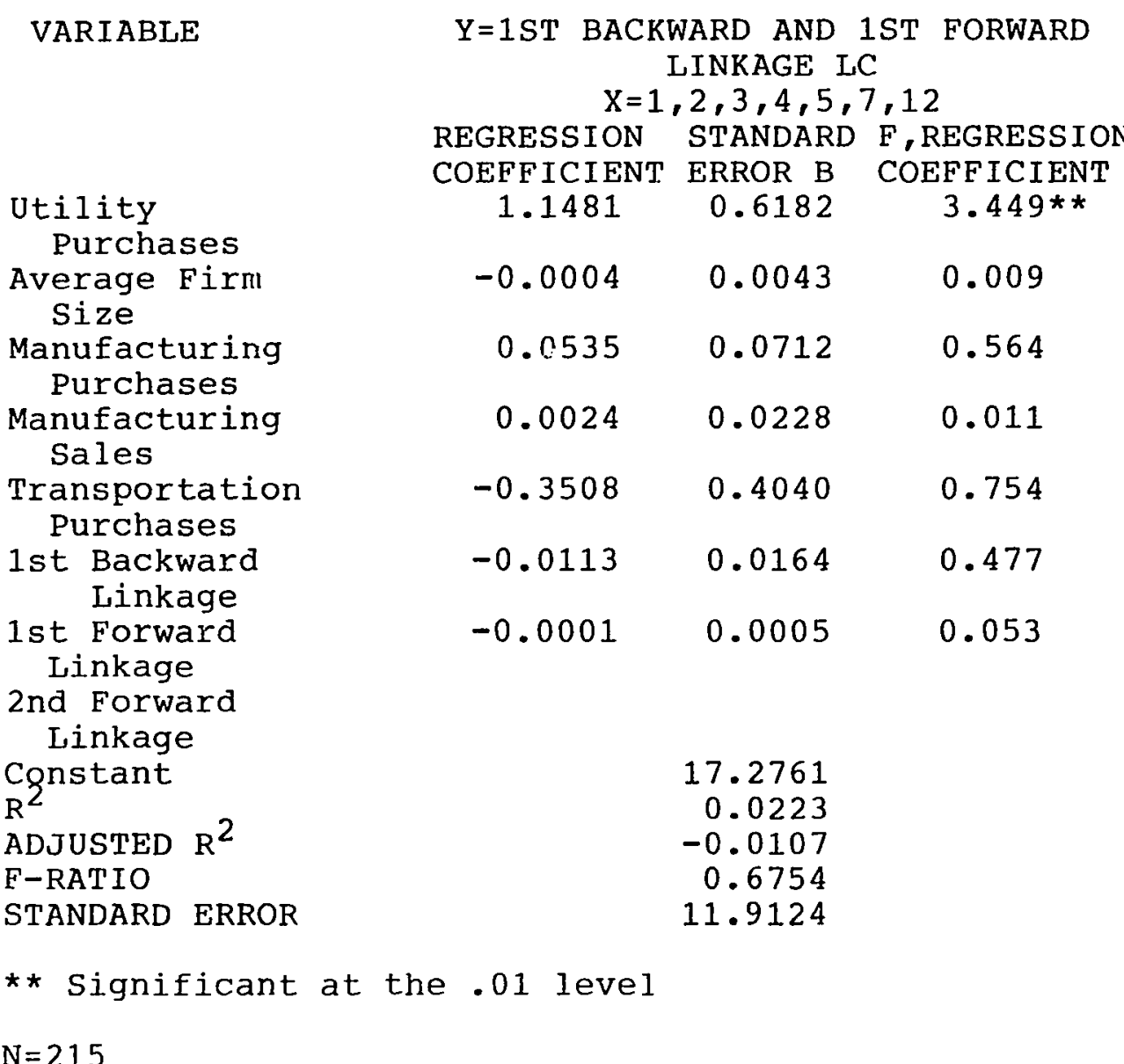

\begin{tabular}{|c|c|c|}
\hline \multicolumn{3}{|c|}{$\begin{array}{l}\mathrm{Y}=1 \mathrm{ST} \text { BACKWARD AND 1ST AND 2ND } \\
\text { FORWARD LINKAGE LC } \\
\mathrm{X}=1,2,3,4,5,7,12,13\end{array}$} \\
\hline REGRESSION & STANDARD & F, REGRESSION \\
\hline $\begin{array}{c}\text { COEFF ICIENT } \\
-1.1214\end{array}$ & $\begin{array}{c}\text { ERROR B } \\
0.9613\end{array}$ & $\begin{array}{c}\text { COEFF ICI IENT } \\
1.361\end{array}$ \\
\hline-0.0047 & 0.0052 & 0.804 \\
\hline 0.0523 & 0.0958 & 0.298 \\
\hline 0.0038 & 0.0313 & 0.015 \\
\hline-0.0520 & 0.5394 & 0.009 \\
\hline-0.0178 & 0.0199 & 0.807 \\
\hline 0.0000 & 0.0006 & 0.001 \\
\hline 0.0739 & 0.0897 & 0.678 \\
\hline & $\begin{array}{r}27.0817 \\
0.0357 \\
-0.0129 \\
0.7350 \\
14.1956\end{array}$ & \\
\hline
\end{tabular}


TABLE XIV

REGRESSIONS OF ONE BACKWARD AND THREE FORWARD LINKAGES AND TWO BACKWARD AND ONE FORWARD LINKAGES WITH COVARIATES

\section{VARIABLE}

Utility

Purchases

Average Firm

$$
\text { Size }
$$

Manufacturing Purchases

Manufacturing

Sales

Transportation

Purchases

1st Backward

Linkage

1st Forward

Linkage

2nd Backward

Linkage

2nd Forward

Linkage

3rd Forward

Linkage

Constant

ADJUSTED $\mathrm{R}^{2}$

F-RATIO

STANDARD ERROR

* Significant at the .05 level

$-0.0083$

0.0177

0.0481

0.8440

$-0.0206$

0.0344

$-0.0563$

0.1037

\section{$\mathrm{Y}=1 \mathrm{ST}$ BACKWARD AND 1ST, 2ND AND \\ THIRD FORWARD LINKAGE LC \\ $X=1,2,3,4,5,7,12,13,14$ \\ REGRESSION STANDARD F, REGRESSION \\ COEFFICIENT ERROR B COEFFICIENT}

$\begin{array}{lll}-2.2026 & 1.4911 & 2.182 *\end{array}$

$0.0075 \quad 1.212$

0.1566

0.013

0.0493

0.954

0.8689

0.944

0.0283

0.530

0.1050

0.107

0.1050

0.1936

0.085

0.4837

0.046

37.0372

0.0424

$-0.0421$

0.5016

19.5740

$\mathrm{N}=112$
$Y=1 S T$ AND 2ND BACKWARD AND 1ST FORWARD LINKAGE LC $\mathrm{X}=1,2,3,4,5,7,8,12$

REGRESSION STANDARD F, REGRESSION COEFFICIENT ERROR B COEFFICIENT

$$
\begin{array}{lll}
1.0334 & 0.8642 & 1.430
\end{array}
$$

$-0.0010$

0.0056

0.030

0.1140

0.0985

1.339

0.0251

0.0308

0.661

$-0.2331$

0.6020

0.150

$-0.0241$

0.0282

0.728

$-0.0002$

0.0006

0.129

0.0312

0.1806

0.030

24.0940

0.0225

$-0.0178$

0.5589

15.7316

$\mathrm{N}=203$ 
TABLE XV

REGRESSIONS OF TWO BACKWARD AND TWO FORWAED LINKAGES AND TWO BACKWARD AND THREE FORWARD LINKAGES WITH COVARIATES

VARIABIE

Utility

Purchases

Average Firm Size

Manufacturing Purchases

Manufacturing Sales

Transportation Purchases

1st Backward Linkage

1st Forward Linkage

2nd Backward Linkage

2nd Forward Linkage

3rd Forward Linkage

Constant

$\mathrm{R}^{2}$

ADJUSTED $\mathrm{R}^{2}$

F-RATIO

STANDARD ERROR $\mathrm{N}=159$
$Y=1 S T$ AND 2ND BACKWARD AND FORWARD LINKAGE LC $X=1,2,3,4,5,7,8,12,13$

REGRESSION STANDARD F, REGRESSION COEFFICIENT ERROR B COEFFICIENT

$$
\begin{array}{lll}
-1.7342 & 1.3248 & 1.713
\end{array}
$$

$\begin{array}{lll}-0.0056 & 0.0067 & 0.715\end{array}$

$\begin{array}{lll}0.1080 & 0.1286 & 0.706\end{array}$

$\begin{array}{lll}0.0275 & 0.0407 & 0.456\end{array}$

$\begin{array}{lll}0.1329 & 0.7974 & 0.028\end{array}$

$-0.0318$

0.0353

0.812

$-0.0000$

0.0008

0.004

0.0658

0.2434

0.073

0.0658

0.1166

0.318

18.1693

0.0375

$-0.0206$

0.6454

18.1693
$\mathrm{Y}=1 \mathrm{ST}$ AND 2ND BACKWARD AND 1ST 2ND AND 3RD FORWARD LINKAGE LC $X=1,2,3,4,5,7,8,12,13,14$ REGRESSION STANDARD $F$, REGRESSION COEFEICIENT ERROR B COEFFICIENT

$$
\begin{array}{lll}
-2.3910 & 2.0219 & 1.398
\end{array}
$$

$\begin{array}{lll}-0.0101 & 0.0092 & 1.223\end{array}$

$\begin{array}{lll}0.0571 & 0.1952 & 0.086\end{array}$

$\begin{array}{lll}0.0735 & 0.0597 & 1.513\end{array}$

$\begin{array}{lll}0.8156 & 1.3071 & 0.389\end{array}$

$\begin{array}{lll}-0.0483 & 0.0543 & 0.791\end{array}$

$\begin{array}{lll}0.0376 & 0.1296 & 0.084\end{array}$

$\begin{array}{lll}0.1908 & 0.4162 & 0.210\end{array}$

$\begin{array}{lll}-0.0373 & 0.2382 & 0.024\end{array}$

$\begin{array}{lll}0.1410 & 0.6015 & 0.055\end{array}$

23.6161

0.0465

$-0.0508$

0.4783

23. 6161

$\mathrm{N}=109$ 
TABLE XVI

REGRESSIONS OF THREE BACKWARD AND ONE FORWARD LINKAGES AND THREE BACKWARD AND TWO FORWARD LINKAGES WITH COVARIATES

\begin{tabular}{|c|c|c|c|c|c|c|}
\hline \multirow[t]{3}{*}{ VARIABLE } & $\begin{array}{r}\mathrm{Y}=1 \mathrm{ST}, \quad 2 \mathrm{ND} \\
1 \mathrm{ST} \text { FOR } \\
\mathrm{X}=1,2,3\end{array}$ & $\begin{array}{l}\text { AND 3RD B } \\
\text { WARD LINK } \\
4,5,7,8,\end{array}$ & $\begin{array}{l}\text { ACKWARD AND } \\
\text { AGE LC } \\
9,12\end{array}$ & $\begin{array}{l}Y=1 S T, \quad 2 N D \\
1 S T \text { AND } 2 N D \\
X=1,2,\end{array}$ & $\begin{array}{l}\text { AND 3RD BA } \\
\text { FORWARD L } \\
3,4,5,7,8,\end{array}$ & $\begin{array}{l}\text { ACKWARD AND } \\
\text { INKAGE LC } \\
9,12,13\end{array}$ \\
\hline & REGRESSION & STANDARD & F, REGRESSION & REGRESSION & STANDARD & F,REGRESSION \\
\hline & COEFFICIENT & ERROR B & COEFF ICIENT & COEFFICIENT & ERROR B & COEFFICIENT \\
\hline $\begin{array}{l}\text { Utility } \\
\text { Purchases }\end{array}$ & 1.0941 & 1.1948 & 0.839 & -1.9361 & 1.6597 & 1.361 \\
\hline Average Firm Size & 0.0009 & 0.0072 & 0.016 & -0.0065 & 0.0079 & 0.670 \\
\hline $\begin{array}{l}\text { Manufacturing } \\
\text { Purchases }\end{array}$ & 0.0141 & 0.1495 & 0.009 & 0.0444 & 0.1782 & 0.062 \\
\hline $\begin{array}{l}\text { Manufacturing } \\
\text { Sales }\end{array}$ & 0.0571 & 0.0546 & 1.092 & 0.0236 & 0.0676 & 0.122 \\
\hline $\begin{array}{l}\text { Transportation } \\
\text { Purchases }\end{array}$ & -0.2731 & 0.8572 & 0.101 & -0.0003 & 1.0511 & 0.000 \\
\hline $\begin{array}{l}\text { 1st Backward } \\
\text { Linkage }\end{array}$ & -0.0968 & 0.1017 & 0.905 & -0.0465 & 0.1176 & 0.156 \\
\hline $\begin{array}{l}\text { 1st Forward } \\
\text { Linkage }\end{array}$ & 0.0827 & 0.0541 & $2.337 *$ & 0.0056 & 0.0876 & 0.004 \\
\hline $\begin{array}{c}\text { 2nd Backward } \\
\text { Linkage }\end{array}$ & 0.4207 & 0.3680 & 1.3307 & 0.7827 & 0.4623 & $2.866 * \star$ \\
\hline $\begin{array}{l}\text { 2nd Forward } \\
\text { Linkage }\end{array}$ & & & & 0.1627 & 0.1689 & 0.929 \\
\hline $\begin{array}{l}\text { 3rd Backward } \\
\text { Linkage }\end{array}$ & -0.6970 & 0.6247 & 1.245 & -1.0336 & 0.7316 & $1.996 *$ \\
\hline $\begin{array}{l}\text { Cqnstant } \\
\mathrm{R}^{2} \\
\text { ADJUSTED } \mathrm{R}^{2} \\
\text { F-RATIO } \\
\text { STANDARD ERROR }\end{array}$ & & $\begin{array}{r}34.3197 \\
0.0489 \\
-0.0074 \\
0.8690 \\
19.9027\end{array}$ & & & $\begin{array}{r}43.3098 \\
0.0597 \\
-0.0207 \\
0.7430 \\
21.2733\end{array}$ & \\
\hline $\begin{array}{l}* \quad \text { Significant at } \\
N=162\end{array}$ & .05 level & & & $\begin{array}{l}\star \star \text { Signific } \\
\mathrm{N}=128\end{array}$ & ant at the & e .01 level \\
\hline
\end{tabular}


TABLE XVII

REGRESSION OF THREE BACKWARD AND THREE FORWARD IINKAGES WITH COVARIATES

\begin{tabular}{|c|c|c|c|}
\hline VARIABLE & $\begin{array}{l}\text { REGRESSION } \\
\text { COEFFICIENT }\end{array}$ & $\begin{array}{l}\text { STANDARD } \\
\text { ERROR B }\end{array}$ & $\begin{array}{l}\text { F, REGRESSION } \\
\text { COEFFICIFNT }\end{array}$ \\
\hline $\begin{array}{l}\text { Utility } \\
\text { Purchases }\end{array}$ & -2.2396 & 2.3859 & 0.881 \\
\hline $\begin{array}{l}\text { Average Firm } \\
\text { Size }\end{array}$ & -0.0135 & 0.0103 & 1.713 \\
\hline $\begin{array}{l}\text { Manufacturing } \\
\text { Purchases }\end{array}$ & -0.0022 & 0.2489 & 0.000 \\
\hline $\begin{array}{l}\text { Manufacturing } \\
\text { Sales }\end{array}$ & 0.1553 & 0.0990 & $2.463 * \star$ \\
\hline $\begin{array}{l}\text { Transportation } \\
\text { Purchases }\end{array}$ & -0.5201 & 1.6012 & 0.106 \\
\hline $\begin{array}{l}\text { 1st Backward } \\
\text { Linkage }\end{array}$ & 0.0006 & 0.1790 & 0.000 \\
\hline $\begin{array}{l}\text { 1st Forward } \\
\text { Linkage }\end{array}$ & 0.0800 & 0.1549 & 0.266 \\
\hline $\begin{array}{l}\text { 2nd Backward } \\
\text { Linkage }\end{array}$ & 1.6468 & 0.7408 & $4.942 * \star *$ \\
\hline $\begin{array}{l}\text { 2nd Forward } \\
\text { Linkage }\end{array}$ & 0.1166 & 0.2800 & 0.173 \\
\hline $\begin{array}{l}\text { 3rd Backward } \\
\text { Linkage }\end{array}$ & -1.3361 & 1.1215 & 1.419 \\
\hline $\begin{array}{l}\text { 3rd Forward } \\
\text { Iinkage }\end{array}$ & 0.4478 & 0.7695 & 0.339 \\
\hline Constant & 37.6609 & & \\
\hline $\begin{array}{l}\mathrm{R}^{2}=0.1325 \quad \text { ADJUSTED } \\
\text { STANDARD ERROR }=26.269 \\
\star \star \star \text { Significant at the }\end{array}$ & $\begin{array}{l}\mathrm{R}^{2}=0.011_{\star \star} \\
.001 \text { level }\end{array}$ & $\begin{array}{c}\text { F-RATIO }=1.0966 \\
\text { Significant at the }\end{array}$ & $\begin{array}{r}.01 \text { level } \\
\mathrm{N}=91\end{array}$ \\
\hline
\end{tabular}


orientation was utilized and the inter-industry 5 th backward linkage. (Tables III and VI). In the former case the experimental variable did not have a significant $F$ ratio while in the latter the experimental variable, the 5 th backward linkage, was significant at the .05 level. Little variability in the dependent variable was accounted for by the covariates and experimental variables in these two runs with multiple coefficients of determination of 0.0508 and 0.2091 respectively.

Statistically significant relationships between experimental variables and the dependent variable existed only for the 5 th backward linkage when intra-industry and inter-industry linkages were considered individually (Table VII). In forward/backward inter-industry linkage combinations the 1st forward linkage was significant at the the 2 nd and $3 r d$ backward linkages in the 3 backward/2 forward run (Table XVI); and the backward linkage in the 3 backward and forward combination (Table XVII).

No consistent pattern emerged between inter-industry firm spatial proximity and the experimental variables with statistically significant regression coefficients. Increases in purchases of inputs from the 5 th inter-industry linkage are associated with a more dispersed array of subject firms and suppliers (Table VII). This tendency towards randomness is also evidenced in the relationship 
between the first forward linkage and firm spatial proximity when considered in combination with the first three backward linkages. As the magnitude of the third backward linkage in an array that includes three backward and two forward linkage firms increases inter-industry firm spatial proximity decreases (Tables XV and XVI). Increases in the purchases of inputs from the third largest supply source results in a more concentrated distribution of firms among the purchaser, three largest suppliers and two manufacturing industry markets (Table XVI). The second largest supply source exerts pressures towards concentration for firm gruunings composed of a subject industry with its three largest suppliers and output buyers (Table XVII). These statistically significant dependent and experimental variable relationships occurred in models with extremely small multiple coefficients of determination. The experimental variables and covariates working together could account for no more than 13 percent of the variability in the dependent variable in any of these applications. The result is statistically significant relationships among a few of the experimental variables and the dependent variable which have little explanatory power. Among the covariates only utility costs, market orientation and average firm size were significant at the 
statistically significant relationships between covariates and intra-industry or inter-jndustry spatial proximity appeared only in applications with extremely small overall multiple coefficients of determination.

Utility costs were positively related to firm spatial proximity for the intra-industry and individual backward linkages and significant at the .01 level or greater for the intra-industry (market orientation covariate), lst and 4 th backward and combination backward linkage runs. The direction of the industry utility intensiveness with spatial proximity for the forwara and backward and forward combinations was not consistent. Percent of industry expenditures spent on utilities was significant at the .05 level or greater for the 2nd forward; 1st, 2nd and 3rd forward comination; and three forward/backward combinations. Utility costs were inversely related to spatial proximity in the 2nd forward, forward combination, and 1 backward/ 3 forward linkage combination models. Utility costs were positively related to the dependent variable in the run which used 1 backward and 1 forward linkage.

Market orientation (percent of manufacturing sales of all sales) was negatively related to spatial proximity at the .05 level of significance for the intra-industry linkage run containing the covariate and the 3rd backward linkage application (Tables IV and VI) In the model which combined 
the first 3 backward and forward linkages market orientation was positively related to the dependent variable at the .01 level (TABLE XVI). Average firm size was positively related to inter-industry firm spatial proximity and significant at the .001 level for the 5 th backward linkage application (Table VII). No consistent patterns were found between average firm size except as specified and transportation costs with the dependent variable for the covariance applications.

\section{REGRESSION FINDINGS AND THE RESEARCH HYPOTHESES}

The first hypothesis anticipated no relationship between intra-industry technological linkages and intraindustry firm spatial proximity. No statistically significant regression coefficients were produced in either of the intra-industry linkage models. The first model used percent of manufacturing inputs to total inputs purchased. In the second application percent of manufacturing outputs to total outputs was substituted for the former in the covariate sequence. Failure to reject the null hypothesis lends support for the research hypothesis of no relationship between intra-industry linkages and intra-industry firm spatial proximity within an urban economy. The second research hypothesis stipulated no 
association between inter-industry backward and forward linkages and related inter-industry firm spatial proximity. The research hypothesis was supported with the retention of the null hypothesis of no difference for the first four backward and all five forward inter-industry technological linkages. An inverse and statistically significant relationship was found between the 5th backward interindustry linkage and inter-industry firm spatial proximity for the subject firm and its 5 th largest supply source. As the percentage of manufacturing inputs from this supplier increased spatial distance between purchasing and supplier firms decreased. However, less than 6 percent of the variability in the dependent variable is attributable to the workings of the covariates and experimental variable in this model.

No association was predicted for the relationship between forward and backward technological linkage combinations and the appropriate combination of interindustry firm spatial array in the third research hypothesis. of the 9 possible combinations for 3 backward and 3 forward inter-industry linkages the null hypothesis was retained in 6 applications. In the remaining models the 1 st forward linkage was positively related to the dependent variable in the 3 backward/l forward combination. The 2nd backward linkage had a statistically significant relationship to the 
dependent variable in a positive direction in the 3 backward/2 forward combination and a negative direction in the 3 backward/3 forward combination. A negative and statistically significant relationship exists between the 3rd backward technological linkage and the dependent variable in the 3 backward/2 forward linkage combination application. In each of these cases less than 14 percent of the variability in inter-industry firm spatial proximity is explained by all covariates and experimental variables in each of the combination applications.

\section{DISCUSSION}

The null hypothesis of no difference for the experimental variables was retained in twenty-one out of twenty-five of the multiple regression covariance applications. This statistical hypothesis was rejected for the 5 th backward linkage in the 5 th backward linkage model; lst forward linkage in the 3 backward/l forward combination run; 2nd and 3rd backward linkages in the 3 backward/2 forward combination; and 2nd backward linkage in the 3 backward/3 forward linkage combination application. Only for the intra-industry linkage (with the market orientation covariate) and the 5 th backward linkage models were statistically significant multiple coefficients of determination produced. In neither application using intraindustry linkages were statistically significant regression 
coefficients produced for the experimental variable. Even in inter-industry applications where statistically significant results were obtained the amount of variability accounted for by the covariates and experimental variabilities working in concert was inconsequential.

The interpretation of results from the applications where the appropriate null hypothesis was retained is relatively straightforward. Technological linkages within an industry and among industries are not determinants of firm location in an urban economy. For the models in which the experimental variables were statistically significant the interpretation is not as clear cut.

In these latter instances if the multiple coefficients of determination had been substantial and the contributions made by the experimental variables relatively large, then variability in the dependent variable could have been accounted for in large measure by the influence of the experimental factors. This was not the case. The multiple coefficients were so low that little variability in the dependent variable could be attributed to the influence of the covariates working in aggregate and/or the experimental variables.

For the statisicically significant experimental variables the question, given their measured lack of impact, 
is whether these results could have been obtained as a result of the variables or statistically procedures utilized. By extension if these factors unduly influenced the results obtained for the statistically significant experimental variables then the same forces would be in operation on the other independent variables used in this study.

The definition and operationalization of the variables in this study were commented on length in the literature review and methodology chapters. To review, input and output coefficients are standardly utilized in the study of economic systems and the inter-relationships of sectors and industries (Leontief 1951; Miernyk 1965; Smith 1981). Following the advice of Tiebout (1956) and Isard (1960) national coefficients were used because of the greater stability of these measures over regional coefficients since the study design is cross-sectional but is the result of a longtitudinal decision-making process. The effects of secondary products have been controlled in the 1972 national transactional tables to provide better information on industry input requirements and input/output relationships (Ritz 1979). Finally within industry homogeneity has been enhanced while between industry heterogeneity is preserved through the use of the standard Industry Classification/ Commodity system at the 4 digit level of disaggregation (Norcliffe 1975). 
Debate on the use of the nearest neighbor procedure focuses on its use as a statistic in the classification of firm spatial arrays and the effect of area size and shape on measurements obtained (Lloyd and Dicken 1972). In this study the use of nearest neighbor is limited to its use as a measure of firm spatial proximity. Area size and shape arguments are not pertinent as the area in this research is fixed and its shape conforms to the Oregon portion of the metropolitan region. It is within these fixed boundaries that the determination of firm spatial proximity is made. The straight line distance between each firm and its nearest neighbor within the defined area is used to calculate the nearest neighbor measure. This method can be followed when investigating firm spatial arrays within an industry. When the array is composed of an industry and its trading partner(s) and the task is to determine the nearest linked industry firm to the subject industry the methodology required by nearest neighbor cannot be followed. It is likely that one or more linked firm will be a -nearest neighbor' to any of the subject firms and will therefore be excluded from consideration. Therefore the requirement that all firms be included is violated and the technique cannot be utilized.

The local concentration index was developed to provide a means for measuring the straight line distance between 
subject industry firms and the closest firm within a linked industry. As with the nearest neighbor the use of the local concentration technique as a measurement circumvents the question of the influence of different area sizes and shapes upon the measurement because of the fixed areal base used in this study. The shape of the distribution of the local concentration measures is relevant to the question of its interpretation as a statistic but not to its more limited use as a measure of spatial distance among two firms within a defined space. The question asked in the study is whether intra-industry and inter-industry linkages can be used to explain tendencies to minimize distance among firms in technologically linked industries. The performance of the covariates in the regression models suggests that the variability observed in the dependent variable can be influenced by variables constructed in the same way as the experimental variables. Inspection of the distribution of scores for the 220 industries used on the independent and dependent variables indicates that lack of score range and variability cannot be used to explain the absence of impact by the selected variables on firm spatial proximity.

The other possibility for lack of significant multiple coefficients of determination other than the research hypotheses is the violation of the assumptions of multiple 
linear regression. These are the use of interval data from a population without bias where each variable is normally distributed and variances equal and the independent variables are orthogonal to each other (Rabiega 1984). Each of these aspects is examined in turn.

Clearly the data is interval level and and cases were drawn from a population without bias. Variances were equalized through the conversion of variable scores into percentages for use in the model. Lack of colinearity among the independent variables was evidenced in the low coefficients exhibited in the variable correlation matrix. The highest correlation coefficient, 0.4353 , was achieved by the composite transportation and utility cost variables. Association values among each other pair of independent variables was less than 0.2900 .

The remaining assumption of normal distribution of variables was violated in this study. Each variable as indicated by the mean and standard deviation had a slight right skew associated with the presence of a few extreme scores. This skew was slight and consistent across all variables in the model with the greatest skew being produced by average firm size. Skews may result in the slight inflation of the $F$ ratio. Since lack of statistically significant multiple coefficients of determination was common in this study it is clear that 
the violation of this assumption was not problematic for the study's outcomes.

The final point which needs addressing in this section is the presence of statistically significant regression coefficients for variables within models without statistically significant $\mathrm{F}$ ratios and very low multiple coefficients of determination. While these results help to substantiate the validity of the model used their substantive importance as locational factors must continue to be questioned when so little variability in the dependent variable can be attributed to their influence. The presence of one or two statistically significant experimental variables within the more complex inter-industry combination applications suggests that more research may be needed into the spatial relationships of manufacturing firms within industrial clusters on a firm to firm basis. As an input the swing in the direction of relationship between industry utility intensiveness and inter-industry firm spatial proximity from positive in the input applications to negative in the forward linkage models is consistent with the expected operation of the model. Both in the case of utilities and transportation the contention by Norcliffe (1975) of the importance of the availability of needed types of infrastructure may be at least as important as cost differentials associated with site selection within $\dot{a}$ 
metropolitan or local level urban economy.

CONCLUSION

The research hypotheses were supported in twenty-one out of twenty-five applications of the model. Intra-industry technological linkages were not found to be important determinants of within industry firm spatial proximity in a metropolitan region. Inter-industry technological linkages were likewise not found to be important locational determinants except in the case of the 5 th backward linkage when considered individually. Statistically significant associations were found between the 1st forward linkage in the 3 backward/1 forward linkage application; the 2nd backward linkage in the 3 backward/2 forward and 3 backward and forward combination runs; and the 3rd backward linkage in the 3 backward/2 forward linkage model. These experimental variables were, however, able to account for little of the variability in the dependent variable when considered individually or working in concert with the covariates in the model. In these latter cases statistical significance was not associated with substantive importance. Alternative hypotheses which wolid have attributed results obtained to the study's design and implementation were examined. Failure to reject the null hypotheses and lack of explanatory power of alternative hypotheses lends 
support for the research hypotheses. Intra-industry and inter-industry technological linkages have not been shown to be important factors in the search for sites by manufacturing industries within a metropolitan economy. 


\section{CHAPTER V}

\section{CONCLUSION}

Manufacturing intra-industry and inter-industry technological linkages are rejected as important determinants of the spatial distribution of manufacturing firms in an urban economy. Spatial proximity of firms in an industry was not found to be related to the magnitude of intra-industry linkages. The strength of primary backward and forward manufacturing linkages could also not be used to explain the degree of spatial concentration found among manufacturing industries in the metropolitan area. There are several implications of these findings for plant location theory. Clearly economies of agglomeration and external economies have no effect on industrial patterns in this analysis. Second, that the poor performance of covariates suggest that application of existing theory to localized manufacturing plant selection processes may be misplaced. Third, that the distinction between economies of agglomeration and external economies may be easily made at the theoretical level but for empirical investigation this differentiation is elusive and questionable. Finally, that a reappraisal of the factors of manufacturing plant site selection at the metropolitan level 
is needed. In this study industry spatial patterns were found to be random not only with respect to linkage variables but also to the traditionally used variables of firm size, manufacturing supply and market dependencies, and transportation and utility cost sensitivity.

The first implication is strongly supported by the current research. It is the major research hypothesis which has been confirmed. These findings do not, however, challenge the viability of 1 inkages as explanatory factors at the larger regional, national, or international levels. Nor do these results question the importance of linkages found at the metropolitan level by studies such as Harrigan and walker between manufacturing industries and other sectors of the economy such as commerce. They simply say in a pure manufacturing location situation at a local level that agglomeration and external economies as elaborated in this research are not operative.

Though no attempt to infer to broader geographical scales the experience here connotes the following for larger scale studies: 1 ) industry disaggregation to the maximum level feasible is advisable; 2) all pertinent variables need to be included in multiple variable or covariate models; and 3 ) there is substantial support for previous research efforts and theoretical frameworks which minimize the importance of manufacturing based linkage agglomerations at the metropolitan level. As stated earlier, 
however, these results do not challenge the influence of inter-sectoral linkages on manufacturing plant location at this scale of development.

The utility of a distinction between intra-industry and inter-industry linkage patterns and the associated differentiation between linkage produced economies of agglomeration and external economies cannot be supported. Industries in this study were classified according to their 4 digit Standard Industrial Classification. Spatial proximity was measured between firms. Even at this level of disaggregation intra-industry technological patterns did not distinguish themselves in performance from interindustry technological linkages. Ordering of intra-industry and forward and backward linkages based on magnitude of the relationship resulted in no systematic positioning of the intra-industry linkages with respect to other principals. The former concept may have utility when reserved to describe transactional patterns in a vertically integrated corporation where savings are generated through the internalization of costs associated with the acquisition of goods and services and to study the site selection process of an individual firm.

Variability in the spatial proximity of firms across manufacturers in the metropolitan area could not be accounted for by manufacturing inter-industry linkages, industrial supply and market orientations, or transportation 
and utility cost sensitivity. While there can be and probably are some individual manufacturing industries where some or all of these variables may have a measurable effect the lack of positive systematic findings across industries suggests that other factors must be used to describe localized plant site patterns.

Norcliffe (1975) contends that infrastructure availability, internal and external economies, contact, and linkages are the most important site locational factors. The Standard Research Institute study of Portland (1982) stressed the need for the maintenance of an inventory of industrial zoned sites with varying mixtures and levels of physical and social infrastructure capable of meeting the needs of diverse locational needs of a multiplicity of industries if recruitment efforts were to be successful. Taken together these studies provide the basis of a list of site locational factors which need to be pursued when linkages are used to describe firm relationships across sectors of the economy. Such an approach would be consistent with the work of Greenhut (1964) and Thomas (1979) who make distinctions among firm, industry, site, geographic area, and socio-political-economic locational factors.

For economic practitioners, industrial recruiters, and planners the study findings suggest that a re-evaluation of policies which stress ties among manufacturers as a primary 
tool for the creation of a new or expanded community employment base should be made. The expenditure of resources on the identification of inter-industry linkages and recruitment of identified target industries may better be spent on the preparation of adequately served developable industrial sites. Such an approach does not, however, negate the need of the community to assess its economic strengths and weaknesses and develop a local strategy likely to attract industries and firms seeking advantages which the community can afford to offer. In weighing the likely impacts of alternative industrial choices on the community careful inspection of estimates of indirect employment impact will obviously need to be made as firm candidates may or may not depend on the local economy for purchase of inputs or sale of products.

Inferred from the study can be support for the use of a joint economic development approach for the communities of a regional economic unit. Linkage relationships are not operative at the localized manufacturing plant site selection level. Spatial proximity to manufacturing linked firms is not required. For industries which are not linkage sensitive local resources can be combined to assure that within the region attractive sites are available. For industries which are linkage sensitive the limited number of sites available in close proximity to other manufacturers can be preserved. The necessary follow-up to this study for 
industrial recruiters is the identification of those specific industries which are dependent upon locations in close spatial proximity to linked firms. 
BIBLIOGRAPHY 


\section{BIBLIOGRAPHY}

Alonso, W., "Location Theory," Regional Development in Planning: A Reader, J. Friedman and W. Alonso, Editors, MIT Press, Cambridge, Massachusetts, 1964.

Auty, R.M., "Small Factors and the Measurement of Internal Economies of Scale," The Professional Geographer, $1975,315-322$.

Baldwin, Robert E., "Industrial Development in Portland," Interviews, Unpublished, 1982.

Battelle Memorial Institute, The Pacific Northwest: A Study of Economic Growth in a Quality Environment, Addendum Report, March 1975 .

Bannock, G., The Economics of Small Firms, Basil Blackwell, Oxford, 1981 .

Bell, Thomas L., Stanley R. Lieber and Gerard Rushton, Clustering of Services," Annals, the Association of American Geographers, 64(2), 1974, 214-225.

Beyers, William B., "On Geographical Properties of Growth Center Linkage Systems," Unpublished Dissertation Draft, University of Washington, circa 1975.

Birch, David L., The Economic Future of City and Suburb", New York: Committee for Economic Development, 1970, 1-15.

Bourne, Larry S., "Private Redevelopment of the Central City," Department of Geography Research Paper, Number 112, University of Chicago, 1967.

Bourne, Larry S., "Urban Structures and Land Use Decisions", Annals, the Association of American Geographers, Vol.66, Number 4, December 1976, 531.

Brown, Robert C., "The Use and Mis-use of Distance Variables in Land Use Analysis," The Professional Geographer, Vol. 20, Number 5, September 1968, 337-341.

Butler, James $W .$, "Use of Input-Output Studies in Industrial Development," AIDC Journal, American Industrial Development Council, Vol. 11, Number 1, January 1976, 39-67.

Chander, Parkash, "The Nonlinear Input-Output Model", Journal of Economic Theory, Vol. 30, Number 2, August 1983, 219-229. 
Clark, P.J. and F.C. Evans, "Distance to Nearest Neighbor as a Measure of Spatial Relationships in Population", Ecology, 35, 1954, 445-453.

Collins, L. and D. F. Walker, Editors, Locational Dynamics of Manufacturing Activity, John Wiley Press, New York, 1975 .

Czamanski, Daniel and Stan Czamanski, "Industrial Complexes: Their Typology, structure, and Relation to Economic Development", Papers, Regional Science Association, $38,1977.93-111$.

Council for Economic Development of Oregon, Development Reporter, Vol. 2, Number 1, 1983.

Ebdon, David, "Spatial Statistics," Statistics in Geography, A Practical Approach, Basic Blackwell, Oxford, $19 \overline{8} 0,106-127$.

Ettlinger, N., "Comments on the Concept of Linkages from the Perspective of Corporate Organization in the Modern Capitalist System," Tijdschrift voor Economische en Sociale Geografie, 75, 1984, 285-291.

Evans, Alan W., "The Pure Theory of City Size in an Industrial Economy," Urban Studies, February 1972, 49-77.

Executive Office of the President, Office of Management and Budget, Standard Industrial Classification Manual 1972, U. S. Government Printing Office, Catalogue Number PREX $2.6 / 2:$ IN $27 / 972,1972$.

First Interstate Bank, "Capital Availability in the Pacific Northwest," Multnomah County Economic Development Advisory Commission, Unpublished Report, winter 1977.

Galbraith, J. K., Economics and the Public Purpose, Penguin Press, Harmondsworth, 1975 .

Getis, Arthur, "On the Use of the Term "Random" in Spatial Analysis," The Professional Geographer, Vol. 29, Number 1, February 1977, 58-61.

Gibson, L. J. and Marshall A. Worden, "Estimating the Economic Base Multiplier: A Test of Alternative Procedures," Economic Geography, 57, 1981, 146-159.

Green, D. Howard, "Industrialists Information Levels of Regional Incentives, Regional Studies, Vol. 11, $1977,7-18$. 
Greenhut, Melvin L., Plant Location in Theory and Practice, University of North Carolina press, Chapel Hill, 1956.

Greenhut, Melvin L., Microeconomics and the Space Economy, Scott Foresman Press, Chicago, $196 \overline{3 .}$

Greenhut, Melvin L., "When Is the Demand Factor of Location Important?", Land Economics, 40, 1964, 175-184.

Greenhut, Melvin L., "Needed--A Return to the Classics in Regional Economic Development Theory," Kyklos, XIX, $1966,461-478$.

Griffith, Daniel A., "A Note on Spatial Autocorrelation," The Professional Geographer, Vol. 27, Number 4, November 1975, 470-474.

Guelke, Leonard, "Regional Geography,". The Professional Geographer, Vo1. 29, Number 1, February 1977, 1-7.

Hakanson, L., "Towards a Theory of Location and Corporate Growth", Spatial Analysis, Industry, and the Industrial Environment, vol. 1, Industrial systems, John Wiley, 1979, 115-138.

Hakimi, S. L., "Optimum Location of Switching Centers and the Absolute Centers and Medians of a Graph", Operations Research, 12, 1964, 450-459.

Harrigan, F. J., "The Relationship between Industrial and Geographical Linkages: A Case Study of the United Kingdom," Journal of Regional Science, Vol. 22, Number 1, 1982, 19-31.

Harvey, D., "The Geography of Capital Accumulation: A Reconstruction of the Marxian Theory," Antipode, Vol. 7, Number 2, 1975, 9-21.

Herr, J. Paul, "Local Governmental Competition for Business Activity: An Urban Case Study," The Professional Geographer, Vol. 31, Number 3, August 1979, 292-298.

Hewings, Geoffrey, "A Note on Forecasting the Economic Base," The Professional Geographer, Vol. 21, Number 5, September 1969, 315-318.

Hewings, Geoffrey, Regional Industrial Analysis and Development, Methuen Press, London 1977.

Hewings, Geoffrey, "The Role of Prior Information in Updating Regional Input-Output Models", SocioEconomic Planning Sciences, Vol. 18, Number 5, 1984, 319-336. 
Holland, S. K., Capital Versus the Regions, Macmillan Press, London, 1976 .

Holland, S. K., The Regional Problem, Macmillan Press, London, $19 \overline{76}$.

Holzner, Lutz, "Urban Geography Without a Regional System," The Professional Geographer, Vol. 18, Number 3, May $1966,129-131$.

Hoover, E. M., Location Theory and the Shoe and Leather Industries, Harvard University Press, Cambridge, Massachusetts, 1937.

Hoover, E. M., The Location of Economic Activity, McGraw-Hi 11 Press, New York, 1948.

Hoover, E. M., "Some Programmed Models for Industry Location", Land Economics, Vol. 43, 1967, 303-311.

Hsu Shin-yi and Clifford E. Tiedemann, "A Rational Method of Delimiting Study Areas for Unevenly Distributed Point Phenomena," The Professional Geographer, Vol. 20, Number 6 , November $6,1968,376-381$.

Hsu, Shin-yi and J. D. Mason, "Towards a Better Understanding of the Boundary Effect on the Decision-Making Process in the Poisson Model of Point Distribution in Bounded Two-Dimensional Space," Proceedings, Association of American Geographers, 4, 1972, 46-49.

Imai, Haruo, "CBD Hypothesis and Economies of Agglomeration", Journal of Economic Theory, Vol. 28, 1982 , 275-299.

Ingram, David R., "An Evaluation of Procedures Utilized in Nearest Neighbor Analysis," Geografiska Annaler, Vol. $60,1978,65-70$.

Isard, W., "Distance Inputs and the Space Economy", Quarterly Journal of Economics, 65, 1951, 181-198.

Isard, W. and Robert E. Kuenne, "The Impact of Steel Upon the Greater New York-Philadelphia Industrial Region: A Study in Agglomeration Projection", Review of Economics and Statistics, 35, 1953.

Isard, W., Location and Space Economy, MIT Press, Cambridge, Massachusetts, 1956.

Isard, W., Methods of Regional Analysis, MIT Press, Cambridge, Massachusetts, 1960. 
Isserman, A. M., "Regional Employment Multiplier: A New Approach: A Comment,". Land Economics, Vol. 51, 1975, 290-293.

Isserman, A. M., "The Location Quotient Approach to Measuring Regional Economic Impacts," Journal of the American Institute of Planners, $19 \overline{77,33-4 \overline{1}}$.

Isserman, A. M., "Estimating Export Activity in a Regional Economy: A Theoretical and Empirical Analysis of Alternate Methods," International Regional Science Review, Vol. 5, 1980, 155-184.

Johnson, Harry G., "The Economic Approach to Social Questions, National Affairs, Vol. 12, i968, 15-23.

Kiel, Stanley, "Input/Output Approach to Predicting Comparative of Industry," Growth and Change, Vol. 11,1980 ,

Kipnis, Baruch A., "Plant Size and Urban Development," Urban Studies, 21, 1984, 53-61.

Lahiri, Sajal, "On Reconciling Purchases and Sales Estimates of a Regional Input-Output Table," Socio-Economic Planning Sciences, Vol. 18, Number 5 , 1984, 337-342.

Lausen, J. R., "On Growth Poles," Urban Studies, Vol. $6,1969,137-161$.

Lemelin, Andre, "Relatedness in the Patterns of Interindustry Diversification," The Review of Economics and Statistjcs, $1982, \overline{646}-656$.

Leontief, W. W., "Input-Output Economics," Scientific American, Vol. 185, 1951, 15-21.

Lichtenberg, R. M., One-tenth of a Nation, Harvard University Press, Cambri $\overline{d g} e$, Massachusetts, 1960.

Lloyd, Peter E. and Peter Dicken, Location in Space, Harper and Row, 1972.

Losch, A., "The Nature of Economic Regions," Southern Economic Journal, Vol. 5, 1938, 71-78.

Louveaux, Francois, Jacques-Francois Thisse and Hubert Beguin, "Location Theory and Transportation Costs:" Regional Science and Urban Economics, Vol. 12, $1982,529-545$.

Mai, Chao-cheng, "Optimum Location and the Theory of the 
Firm Under Demand Uncertainity," Regional Science and Urban Economics, Vol. 11, 1981, 549-557.

Mai, Chao-Cheng, "Demand Function and Location Theory of the Firm Under Price Uncertainity", Urban Studies, 21, 1984, 459-464.

Mathur, V. K., "Location Theory of a Firm Under Price Uncertainity," Regional Science and Urban Economics, Vol. $\overline{11,1981 / 82,529-54} 7$.

Miernyk, W., The Elements of Input-Output Analysis, Random House Press, New York, 1965.

Moomaw, Ronald, "Firm Location and City Size: Reduced Productivity Advantages as a Factor in the Decline of Manufacturing in Urban Areas", Journal of Urban Economics, 17, 1985, 73-79.

Moore, C. L., "A New Look at the Minimum Requirements Approach to Regional Economic Analysis", Economic Geography, $51,1975,350-356$.

Moses, Leon and Harold F. Williamson, Jr., "The Location of Economic Activity in Cities," American Economic Review, Papers and Proceedings, May 1967, 211-222.

Multnomah County, Overall Economic Development Plan I, Colleen Greer Acres, Project Director, Multnomah County Department of Environmental Services, Division of Planning and Development, 1977.

Norcliffe, G. B., "Using Location Quotients to Estimate the Economic Base and Trade Flows," Regional Studies, Vol. 17, 1983, 161-168.

Norcliffe, G. B., "A Theory of Manufacturing Places", Locational Dynamics of Manufacturing Activity, L. Collins and D. F. Walker, Editors, John Wiley Press, New York, 1975, 19-57.

North, Douglas D., "Location Theory and Regional Economic Growth," Journal of Political Economy, Vol. 63, $1955,240-255$.

Nourse, H. O., Regional Economics, McGraw-Hill Press, New York, 1968 .

O'Farrell, P. N., "Employment Change in Manufacturing: The Case of Surviving Plants", Urban Studies, 22, $1985,57-68$.

Openshaw, S., "Ecological Fallacies and the Analysis of 
Areal Census Data", Environment and Planning $\underline{A}$, Vol. 16, 1984, 17-31.

Oregon Legislature, Senate Bill 2295, 62nd Oregon Legislative Assembly, 1983.

Oregon Legislature, Senate Bill 534, 62nd Oregon Legislative Assembly, 1983.

Oregon Department of Land Conservation and Development, Statewide Land Use Planning Goals and Guidelines, 1983 revised.

Ostrom, Vincent, Charles M. Tiebout and Robert Warren, "The Organization of Government in Metropolitan Areas: A Theoretical Inquiry," The Organization of Metropolitan Government, $1960,542-553$.

Perroux, F., "Economic Space Theory and Applications," Quarterly Journal of Economics, Vol. 64, 1950, 89-104.

Perroux, F., "Note on the Concept of "Growth Poles"," Regional Economics, David I. McKee, Robert D. Dean, and William H. Leahy, Editors, The Free Press, 1970, 93-103.

Peschel, Karin, "International Trade and Industrial Location", Regional Science and Urban Economics, Vol. 12, 1982 247-269.

Rabiega, William A., Straight Talk About Statistical Analysis, Unpublished, 1985.

Rees, J., "Decision-making: The Growth of the Firm and the Business Environment", Spatial Perspectives on Industrial organization and Decision-making, $\bar{F}$. E. I. Hamilton, Editor, John Wiley Press, London, 189-212.

Richardson, H., Regional Economics, Weidenfeld and Nicolson press, London, 1969.

Richardson, H., Input-Output Analysis and Regional Economies, John Wiley press, 1972, pp. 85-138.

Ritz, Philip M., "The Input-Output Structure of the U. S. Economy, 1972," Survey of Current Business, Vol. 59, Number 2, February 1979, 34-72.

Romanoff, Eliahu, "Interindustry Analysis for Regional Growth and Development: The Dynamics of Manpower Issues", Socio-Economic Planning Sciences, Vol. 18, Number $5,1984,353-363$. 
Rose, Adam, "Technological Change and Input-Output Analysis: An Appraisal", Socio-Economic Planning Sciences, Vol. 18, Number $\overline{5,1984,305-3} 1 \overline{\text {. }}$

Samuelson, P. A., "Intertemporal Price Equilbrium: A Prologue to the Theory of Speculation", Weltwirtschaftliches Archives, Vol. 79, 1957, 181-221.

Schmidt, C. G., "Spatial Structure of Industrial Linkages and Regional Economic Growth: An Analysis of Linkage Stability Among Pacific Northwest Steel Firms, 19631970, Unpublished Dissertation, University of Washington, Seattle, 1973.

Smith, David M., Industrial Location, An Economic Geographical Analysis, Second Edition, John wiley and Sons Inc., New York, Chichester, Brisbane, Toronto, 1981.

Sommers, Gail, "The Effects of Economic Shifts: How a Small City Copes," Urban Affairs Annual Assocation Meeting Paper, Portland Oregon, March 1984.

Stabler, J. C., "Exports and Evolution: The Process of Regional Change", Land Economics, XIIV, Number 1, $1968,11-23$.

Stanford Research Institute, "Strengths and Weaknesses of the Portland Area as an Industrial Location", Stanford Research Institute, Phase I Report, July 1982.

Streit, M. E., "Spatial Associations and Economic Linkages between Industries", Journal of Regional Science, $9,1969,177-188$.

Swoveland, C., "A Deterministic Multi-Period Production Planning Model with Piecewide Concave Production and Holding Backorder Costs," Management Science, Vol. 21, 1975, 1007-1013.

Tauchen, Helen and Ann D. Witte, "Office Building Costs and Location," Land Economics, Vol. 59, University of Wisconsin Press, 1983, 324-336.

Taylor, M. J., "Organizational Growth, Spatial Interaction and and Location Decision-making," Regional Studies, Vol. $91,1979,313-323$.

Thomas, M. D., "Explanatory Frameworks for Growth and Change in Uninational and Multinational Firms," Commission of Industrial Systems, 1979 Symposium, Rotterdam, Unpublished Conference Paper. 
Thomas, Wilbur R., "Internal and External Factors in the Development of Urban Economies," Issues in Urban Economics, H. S. Perloff and L. Wingo, Editors, John Hopkins Press, Baltimore, 1975, 43-62.

Tiebout, C. M., "Exports and Regional Economic Growth," Journal of Political Economy, Vol. 64, 1956, 256-260.

Tiebout, C. M., "Regional and Interregional Input-Output Models; An Appraisal," Southern Economic Journal, Vol. 24, 1957, 140-147.

Tiebout, C. M., The Community Economic Base Study, Committee for Economic Development, Supplementary Paper, Number 16, December 1962.

Tomer, John F., "Community Control and the Thecry of the Firm. Review of Social Economics, Vol. 38, 1980, 191.

U. S. Department of Commerce, Industrial Location Detriments 1971-1975, William $W$. Blunt Jr., Acting Assistant secretary for Economic Development, February 1973.

Vernon, R., Metropolis 1985, Harvard University Press, Cambridge, Massachusetts, 1960.

Walker, Susanne R., "Linkage Structures in an Urban Economy", Regional Studies, Vol. 11, 1977, 263-273.

Webber, M. J.., The Impact of Uncertainity on Location, MIT Press, Cambridge, Massachusetts, 1972 .

Weber, A., Alfred Weber's Theory of the Location of Industries, translated by C. J. Friedrich, from uber den Standort der Industrien 1909, University of Chicago Press, Chicago, 1929.

White, Michelle J., "Firm Suburbanization and Urban Subcenters", Journal of Urban Economics, 3, 1976, $323-343$.

Wood, P. A., "Industrial Location and Linkage", Area, 2, $1969,32-39$.

Wood, P. A., "Are Behavioral Approaches to Industrial Location Theory Doomed to be Descriptive?," Industrial Alternative Frameworks, D. B. Massey and W. I. Morrison Editors, Center for Environmental Studies, London, 1975, 41-48. 
APPENDIX 
TABLE XVIII

STATUS OF INDUSTRIES WITH ONE FIRM

A. INDUSTRIES USED AS BACKWARD AND FORWARD LINKAGES

\begin{tabular}{|c|c|c|c|}
\hline $\begin{array}{l}\text { INPUT- } \\
\text { OUTPUT } \\
\text { CIASS. } \\
\text { NUMBER }\end{array}$ & $\begin{array}{l}\text { STANDARD } \\
\text { INDUSTRIAL } \\
\text { CLASS. } \\
\text { NUMBER }\end{array}$ & INDUSTRY TITLE & $\begin{array}{l}\text { NUMBER } \\
\text { OF } \\
\text { EMPLOYEES }\end{array}$ \\
\hline 14.2500 & 2075 & Soybean Oil Mills & 2 \\
\hline 14.2900 & 2079 & Shortening and Cooking oils & 114 \\
\hline 14.2101 & 2082 & Malt Liquors & 262 \\
\hline 17.1002 & 2299 & Textile Goods n.e.c. & 45 \\
\hline 27.0201 & 2873 & $\begin{array}{l}\text { Nitrogenous and Phosphatic } \\
\text { Fertilizers }\end{array}$ & 1 \\
\hline 38.0400 & 3334 & Primary Aluminum & 992 \\
\hline 38.0800 & 3354 & Aluminum Rolling and Drawing & 16 \\
\hline 38.1000 & 3357 & $\begin{array}{l}\text { Nonferrous wire Drawing and } \\
\text { Insulation }\end{array}$ & 15 \\
\hline 37.0402 & 3399 & Primary Metal Products n.e.c. & 5 \\
\hline 13.0600 & 3482 & Small Arms Ammunition & 5 \\
\hline 54.0500 & 3635 & Household Vacuum Cleaners & 6 \\
\hline 56.0200 & 3652 & Phonographic Record and Tape & 6 \\
\hline 57.0100 & 3672 & Electron Tubes & 8 \\
\hline 59.0301 & 3711 & Motor Vehicles & 3,358 \\
\hline 64.0302 & 3942 & Dolls & 5 \\
\hline 64.0504 & 3955 & $\begin{array}{l}\text { Carbon Paper and Inked } \\
\text { Ribbons }\end{array}$ & 26 \\
\hline
\end{tabular}


TABLE XVIII (CON'T)

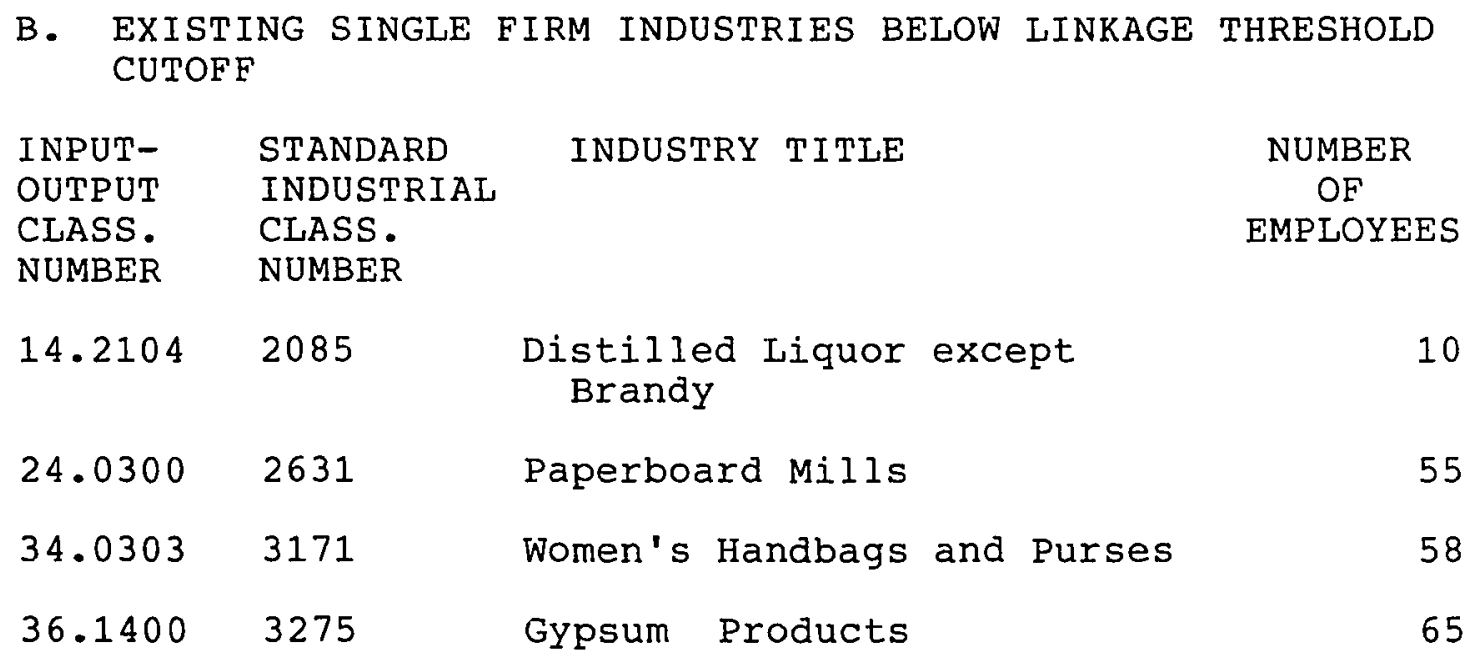


TABLE XIX

INDUSTRY REVISIONS

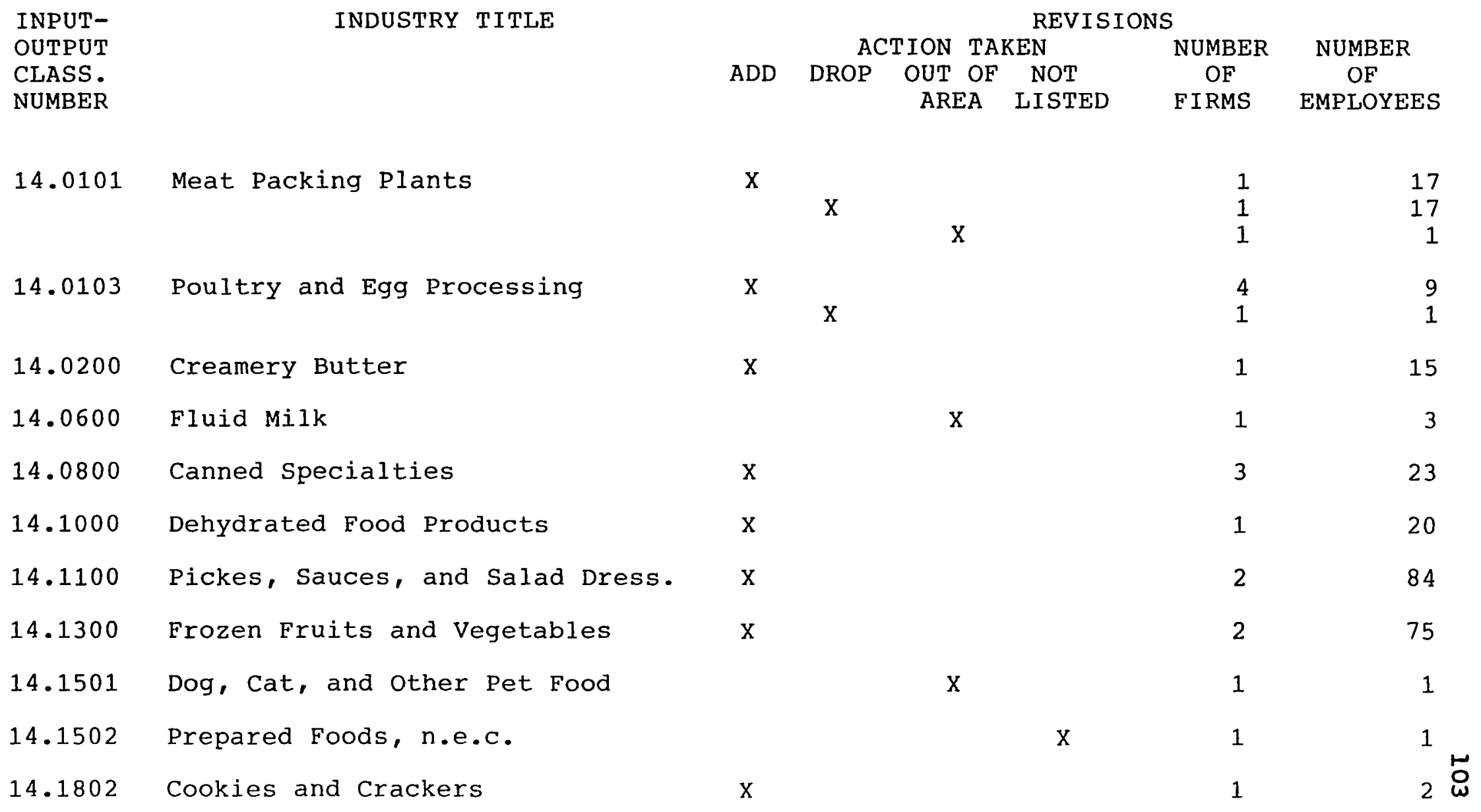


TABLE XIX (CON'T)

\begin{tabular}{|c|c|c|c|c|c|c|c|}
\hline \multirow{4}{*}{$\begin{array}{l}\text { INPUT- } \\
\text { OUTPUT } \\
\text { CIASS. } \\
\text { NUMBER }\end{array}$} & \multirow[t]{4}{*}{ INDUSTRY TITLE } & \multicolumn{6}{|c|}{ REVISIONS } \\
\hline & & \multirow{3}{*}{ ADD } & \multicolumn{3}{|c|}{ ACTION TAKEN } & \multirow{3}{*}{$\begin{array}{l}\text { NUMBEP } \\
\text { OF } \\
\text { FIRMS }\end{array}$} & \multirow{3}{*}{$\begin{array}{c}\text { NUMBER } \\
\text { OF } \\
\text { EMPLOYEES }\end{array}$} \\
\hline & & & DROP & OUT OF & NOT & & \\
\hline & & & & AREA & LISTED & & \\
\hline 14.2021 & Confectionery Products & $\mathrm{X}$ & & & & 3 & 11 \\
\hline & & & & & & & \\
\hline 14.2700 & Animal and Marine Fats and Oils & $X$ & & & & 2 & 27 \\
\hline & & & $\mathrm{X}$ & & & 1 & 17 \\
\hline 14.2103 & Wines, Brandy and Brandy Spirits & $\mathrm{X}$ & & & & 4 & 15 \\
\hline & & & $\mathrm{x}$ & & & 1 & 3 \\
\hline 14.2200 & Bottled and Canned Soft Drinks & $\mathrm{x}$ & & & & 2 & 255 \\
\hline 14.0700 & Canned and Cured Seafoods & $\mathrm{x}$ & & & & 2 & 32 \\
\hline 14.2800 & Roasted Coffee & $\mathrm{x}$ & & & & 1 & 40 \\
\hline 14.3000 & Manufactured Ice & $\mathrm{x}$ & & & & 4 & 56 \\
\hline 14.3100 & Macaroni and spaghetti & $\mathrm{x}$ & & & & 1 & 45 \\
\hline 14.3200 & Food Preparations & $\mathrm{x}$ & & & & 2 & 220 \\
\hline 16.0100 & Broadwoven Fabric Mills & & $\mathrm{x}$ & & & 1 & 240 \\
\hline 19.0301 & Textile Bags & $\mathrm{x}$ & & & & 1 & 8 \\
\hline
\end{tabular}


TABI:E XIX (CON'T)

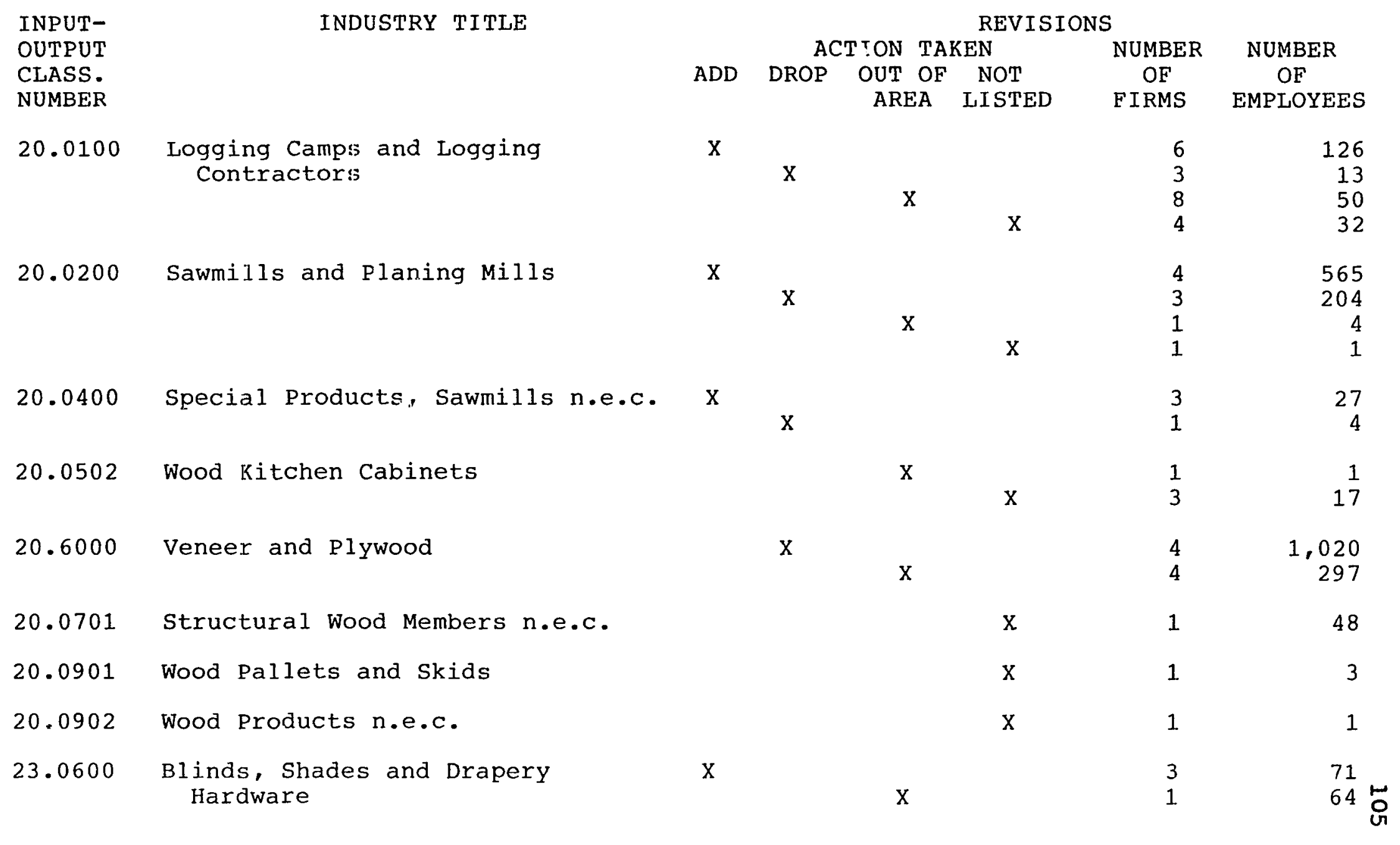


TABLE XIX (CON'T)

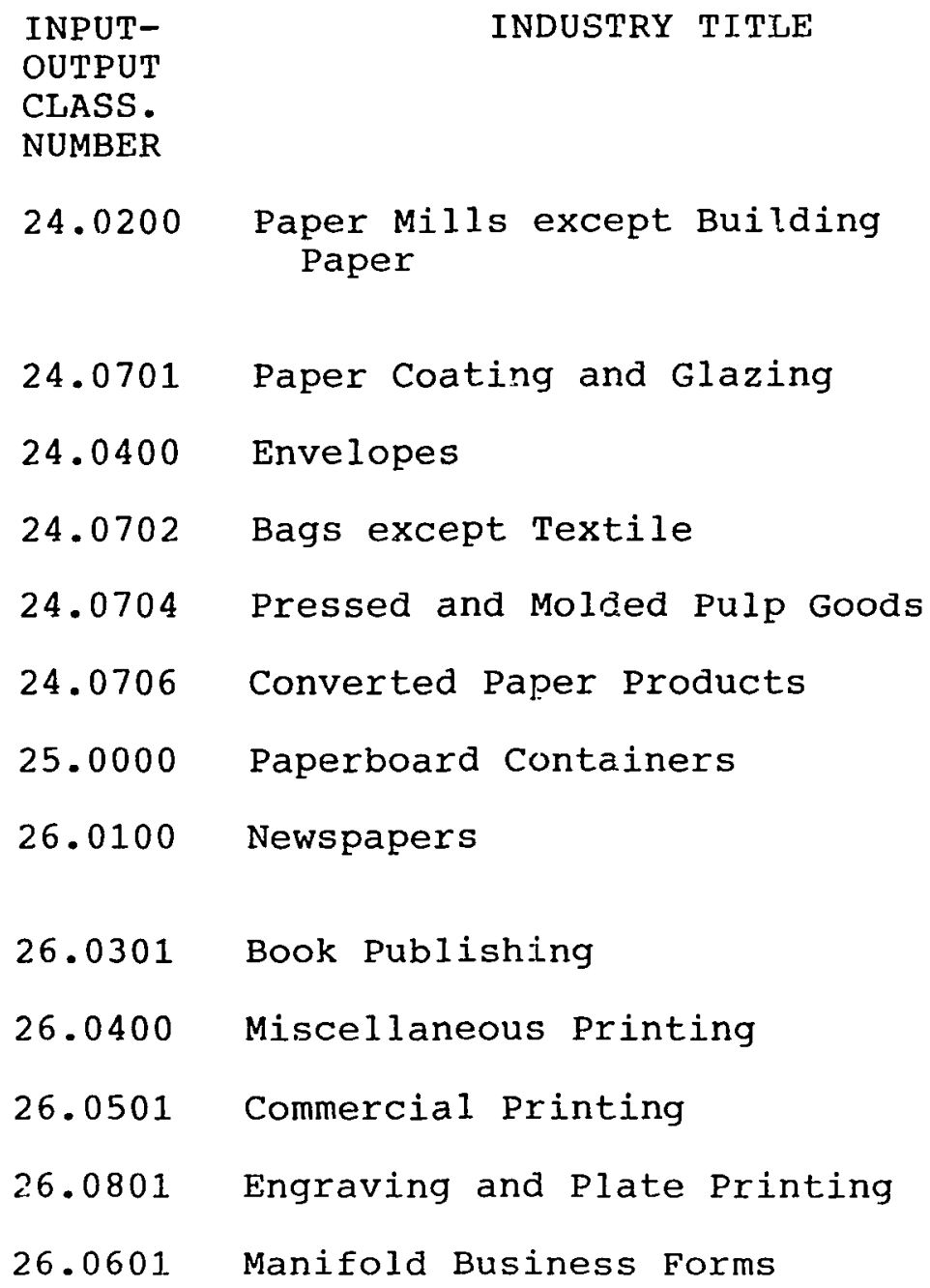

\begin{tabular}{|c|c|c|c|c|c|}
\hline \multicolumn{6}{|c|}{ REVISIONS } \\
\hline \multirow[b]{2}{*}{ ADD } & \multicolumn{2}{|c|}{ ACTION } & TAKEN & \multirow{2}{*}{$\begin{array}{l}\text { NUMBER } \\
\text { OF } \\
\text { FIRMS }\end{array}$} & \multirow{2}{*}{$\begin{array}{c}\text { NUMBER } \\
\text { OF } \\
\text { EMPLOYEES }\end{array}$} \\
\hline & DROP & $\begin{array}{c}\text { OUT OF } \\
\text { AREA }\end{array}$ & $\begin{array}{c}\text { NOT } \\
\text { LISTED }\end{array}$ & & \\
\hline $\mathrm{X}$ & & & & 2 & 106 \\
\hline & $\mathrm{x}$ & & & 1 & 354 \\
\hline & & $\mathrm{X}$ & & 2 & 429 \\
\hline $\mathrm{X}$ & & & & 1 & 5 \\
\hline $\mathrm{X}$ & & & & 1 & 275 \\
\hline $\mathrm{X}$ & & & & 1 & 60 \\
\hline $\mathrm{x}$ & & & & 1 & 56 \\
\hline $\mathrm{X}$ & & & & 2 & 73 \\
\hline & & $\mathrm{x}$ & & 1 & 2 \\
\hline $\mathrm{x}$ & & & & 6 & 243 \\
\hline & & & $\mathrm{x}$ & 2 & 181 \\
\hline & & $\mathrm{X}$ & & 1 & 2 \\
\hline & & $\mathrm{X}$ & & 1 & 2 \\
\hline & & & $\mathrm{X}$ & 5 & 45 \\
\hline $\mathrm{X}$ & & & & 2 & 8 \\
\hline & & & $\mathrm{X}$ & 1 & 28 \\
\hline
\end{tabular}


TABLE XIX (CON'T)

\begin{tabular}{|c|c|c|c|c|c|c|c|}
\hline \multirow{4}{*}{$\begin{array}{l}\text { INPUT- } \\
\text { OUTPUT } \\
\text { CLASS. } \\
\text { NUMBER }\end{array}$} & \multirow[t]{4}{*}{ INDUSTRY TITLE } & \multicolumn{6}{|c|}{ REVISIONS } \\
\hline & & \multirow{3}{*}{ ADD } & \multicolumn{3}{|c|}{ ACTION TAKEN } & \multirow{3}{*}{$\begin{array}{l}\text { NUMBER } \\
\text { OF } \\
\text { FIRMS }\end{array}$} & \multirow{3}{*}{$\begin{array}{c}\text { NUMBER } \\
\text { OF } \\
\text { EMPLOYEES }\end{array}$} \\
\hline & & & DROP & OUT OF & NOT & & \\
\hline & & & & & & & \\
\hline 26.0804 & Photoengraving & $\mathrm{x}$ & & & & 2 & 32 \\
\hline 26.0502 & Lithographic Plate Making and & $\mathrm{x}$ & & & & 2 & 14 \\
\hline 28.0100 & Plastics Material and Resins & $\mathrm{x}$ & & & & 2 & 20 \\
\hline 27.0202 & Fertilizers & $\mathrm{x}$ & & & & 1 & 12 \\
\hline & & & $\mathrm{x}$ & & & 1 & 20 \\
\hline 27.0300 & Agricultural Chemicals n.e.c. & $\mathrm{X}$ & & & & 4 & 116 \\
\hline 27.0402 & Adhesives and Sealants & & & $\mathrm{x}$ & & 1 & 7 \\
\hline 27.0404 & Printing Inks & $\mathrm{x}$ & & & & 1 & 1 \\
\hline & & & & $\mathrm{x}$ & & 1 & 1 \\
\hline 27.0406 & Chemical Preparations n.e.c. & $\mathrm{x}$ & & & & 1 & 200 \\
\hline 31.0100 & $\begin{array}{l}\text { Petroleum Refining and Misc. } \\
\text { Products of Petroleum and Coal }\end{array}$ & $\mathrm{x}$ & & & & 1 & 17 \\
\hline 32.0100 & Tires and Innertubes & $\mathrm{x}$ & & & & 1 & 45 \\
\hline 32.0500 & $\begin{array}{l}\text { Rubber and Pastic Hose and } \\
\text { Belting }\end{array}$ & $\mathrm{x}$ & & & & 1 & 15 \\
\hline 32.0400 & Misc. Plastic Products & & & $\mathrm{X}$ & $\mathrm{X}$ & $\begin{array}{l}3 \\
3\end{array}$ & $\begin{array}{r}18 \text { } \\
8\end{array}$ \\
\hline
\end{tabular}


TABLE XIX (CON'T)

\begin{tabular}{|c|c|c|c|c|c|c|c|}
\hline \multirow{3}{*}{$\begin{array}{l}\text { INPUT- } \\
\text { OUTPUT } \\
\text { r.LASS. } \\
\text { iNUMBER }\end{array}$} & \multirow[t]{3}{*}{ INDUSTRY TITLE } & \multicolumn{6}{|c|}{ REVISIONS } \\
\hline & & \multicolumn{4}{|c|}{ ACTION TAKEN } & \multirow{2}{*}{$\begin{array}{l}\text { NUMBER } \\
\text { OF } \\
\text { FIRMS }\end{array}$} & \multirow{2}{*}{$\begin{array}{c}\text { NUMBER } \\
\text { OF } \\
\text { EMPLOYEES }\end{array}$} \\
\hline & & ADD & DROP & OUT OF & NOT & & \\
\hline 33.0001 & Leather Tanning and Finishing & $x$ & & & & 1 & 2 \\
\hline & & & & $x$ & & 1 & 6 \\
\hline & & & & & $\mathrm{x}$ & 1 & 3 \\
\hline 34.0301 & Leather Gloves and Mittens & $\mathrm{x}$ & & & & 1 & 28 \\
\hline 34.0305 & Leather Goods n.e.c. & $\mathrm{x}$ & & & & 5 & 78 \\
\hline 35.0100 & $\begin{array}{l}\text { Glass and Glass Products except } \\
\text { Contain } 2 \text { rs }\end{array}$ & & & $\mathrm{x}$ & & 2 & 13 \\
\hline 35.0200 & Glass Containers & $\mathrm{x}$ & & & & 1 & 1 \\
\hline 36.0200 & Brick and Structural Clay Tile & $\mathrm{x}$ & & & & 2 & 63 \\
\hline 36.0500 & Structural clay Products n.e.c. & & $\mathrm{x}$ & & & 1 & 13 \\
\hline & & & & $\mathrm{x}$ & & 1. & 19 \\
\hline 36.0900 & Pottery Products & $\mathrm{x}$ & & & & 1 & 60 \\
\hline 36.1000 & Concrete Block and Brick & & & & $\mathrm{x}$ & 1 & 28 \\
\hline 36.1100 & Concrete Products & & & & $\mathrm{x}$ & 1 & 27 \\
\hline 36.1200 & Ready Mix Concrete & $\mathrm{x}$ & & & & 2 & 20 \\
\hline 36.1300 & Lime & $\mathrm{x}$ & & & & 1 & 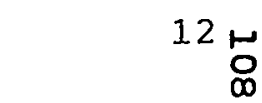 \\
\hline
\end{tabular}


TABLE XIX (CON'T)

$\begin{array}{ll}\begin{array}{l}\text { INPUT- } \\ \text { OUTPUT } \\ \text { CLASS. } \\ \text { NUMBER }\end{array} & \text { INDUSTRY TITLE } \\ 36.1500 & \text { Cut Stone and Stone Products } \\ 36.1600 & \text { Abrasive Products } \\ 36.1700 & \text { Asbestos Products } \\ 36.1800 & \text { Gaskets Packing and Sealing } \\ 36.1900 & \text { Minerals, Ground or Treated } \\ 36.2000 & \text { Mineral Wool } \\ 37.0101 & \text { Blast Furnace and Steel Mills } \\ 37.0105 & \text { Steel Pipes and Tubes } \\ 37.0200 & \text { Iron and Steel Foundaries } \\ 38.0400 & \text { Primary Aluminum } \\ 38.0600 & \text { Secondary Nonferrous Metals } \\ 38.1300 & \text { Nonferrous Castings n.e.c. } \\ 37.0401 & \text { Metal Heat Treating }\end{array}$

\begin{tabular}{|c|c|c|c|c|c|}
\hline \multicolumn{6}{|c|}{ REVISIONS } \\
\hline \multirow[b]{2}{*}{ ADD } & \multicolumn{3}{|c|}{ ACTION TAKEN } & NUMBER & NUMBER \\
\hline & DROP & OUT OF & $\begin{array}{l}\text { NOT } \\
\text { STED }\end{array}$ & $\underset{O F}{O F}$ & OF \\
\hline $\mathrm{x}$ & & & & 2 & 8 \\
\hline $\mathrm{X}$ & & & & 2 & 10 \\
\hline & & $\mathrm{x}$ & & 1 & 1 \\
\hline $\mathrm{X}$ & & & & 2 & 8 \\
\hline $\mathrm{x}$ & & & & 1 & 18 \\
\hline $\mathrm{X}$ & & & & 12 & 639 \\
\hline & & $\mathrm{X}$ & & 1 & 3 \\
\hline $\mathrm{X}$ & & & & 1 & 8 \\
\hline $\mathrm{X}$ & & & & 2 & 142 \\
\hline & $\mathrm{X}$ & & & 1 & 156 \\
\hline $\mathrm{X}$ & & & & 1 & 120 \\
\hline & & $\mathrm{X}$ & & 1 & 1 \\
\hline & & & $\mathrm{X}$ & 1 & 4 \\
\hline$x$ & & & & 1 & 150 \\
\hline $\mathrm{X}$ & & & & 2 & 125 \\
\hline $\mathrm{x}$ & & & & 1 & 3 \\
\hline
\end{tabular}


TABLE XIX (CON'T)

\begin{tabular}{|c|c|c|c|c|c|c|c|}
\hline INPUT- & INDUSTRY TITLE & & & & REVISI & & \\
\hline OUTPUT & & & & IION TAK & KEN & NUMBER & NUMBER \\
\hline CLASS. & & ADD & DROP & OUT OF & NOT & OF & OF \\
\hline NUMBER & & & & AREA & LISTED & FIRMS & EMPLOYEES \\
\hline 39.0100 & Metal Cans & & & $\mathrm{x}$ & & 1 & 5 \\
\hline 39.0200 & Metal Barrels, Drums, and Pails & $\mathrm{x}$ & & & & 2 & 55 \\
\hline 42.0201 & Hand and Edge Tools n.e.c. & & & & $\mathrm{x}$ & 1 & 1 \\
\hline 40.0200 & $\begin{array}{l}\text { Plumbing Fixtures, Fittings, } \\
\text { and Trim }\end{array}$ & $\mathrm{x}$ & & & & 1 & 6 \\
\hline 40.0300 & Heating Equipment except & $\mathrm{x}$ & & & & 2 & 375 \\
\hline & Electrical & & & $\mathrm{X}$ & $\mathrm{X}$ & $\begin{array}{l}1 \\
3\end{array}$ & $\begin{array}{l}24 \\
12\end{array}$ \\
\hline 40.0500 & Metal Doors, Sash, and Trim & & & & $\mathrm{X}$ & 1 & 6 \\
\hline 40.0700 & Sheet Metal Work & $\mathrm{x}$ & & & & 9 & 133 \\
\hline & & & & $\mathrm{x}$ & & 1 & 3 \\
\hline & & & & & $\mathrm{x}$ & 1 & 6 \\
\hline 40.0901 & Prefabricated Metal Buildings & $\mathrm{x}$ & & & & 2 & 6 \\
\hline 41.0100 & $\begin{array}{l}\text { Screw Machine Products and Bolts, } \\
\text { Nuts, Rivets, and Washers }\end{array}$ & $\mathrm{x}$ & & & & 3 & 90 \\
\hline 37.0300 & Iron and Steel Forgings & $\mathrm{x}$ & & & & 1 & 35 \\
\hline 42.0402 & $\begin{array}{l}\text { Metal Coating and Allied } \\
\text { Services }\end{array}$ & $\mathrm{x}$ & & & & 1 & 135 占 \\
\hline
\end{tabular}


TABLE XIX (CON'T)

\begin{tabular}{|c|c|c|c|c|c|c|c|}
\hline \multirow{4}{*}{$\begin{array}{l}\text { INPUT- } \\
\text { OUTPUT } \\
\text { CLASS. } \\
\text { NUMBER }\end{array}$} & \multirow[t]{4}{*}{ INDUSTRY TITLE } & \multicolumn{6}{|c|}{ REVISIONS } \\
\hline & & \multirow{3}{*}{$\mathrm{ADD}$} & \multicolumn{3}{|c|}{ ACTION TAKEN } & \multirow{3}{*}{$\begin{array}{l}\text { NUMBER } \\
\text { OF } \\
\text { FIRMS }\end{array}$} & \multirow{3}{*}{$\begin{array}{l}\text { NUMBER } \\
\text { OF } \\
\text { EMPLOYEES }\end{array}$} \\
\hline & & & DROP & OUT OF & NOT & & \\
\hline & & & & & & & \\
\hline 13.0500 & Small Arms & $\mathrm{x}$ & & & & 1 & 1 \\
\hline 42.0700 & Steel Springs except wire & $\mathrm{x}$ & & & & 1 & 12 \\
\hline 42.0800 & Pipe Valves and Fittings & & & & $\mathrm{x}$ & 1 & 2 \\
\hline 42.1100 & Fabricated Metal Products n.e.c. & $\mathrm{x}$ & & & & 2 & 5 \\
\hline 44.0001 & Farm Machinery and Equipment & $\mathrm{x}$ & & & & 2 & 13 \\
\hline & & & & & $\mathrm{x}$ & 1 & 2 \\
\hline 44.0002 & Lawn and Garden Equipment & $\mathrm{x}$ & & & & 1 & 5 \\
\hline 45.0200 & Mining Machinery except oil & $\mathrm{x}$ & & & & 1 & 4 \\
\hline 46.0100 & Elevators and Moving Stairways & $\mathrm{x}$ & & & & 1 & 12 \\
\hline 46.0300 & Hoist.s, Cranes, and Monorails & $\mathrm{x}$ & & & & 2 & 155 \\
\hline 46.0400 & Industrial Trucks and Tractors & $\mathrm{x}$ & & & & 3 & 12 \\
\hline 47.0100 & $\begin{array}{l}\text { Machine Tools, Metal cutting } \\
\text { Types }\end{array}$ & $\mathrm{X}$ & & & & 2 & 16 \\
\hline 47.0401 & Power Driven Hand Tools & $\mathrm{x}$ & & & & 1 & 17 \\
\hline 48.0100 & Food Products Machinery & $\mathrm{x}$ & & & & 1 & \\
\hline
\end{tabular}


TABLE XIX (CON'T)

\begin{tabular}{|c|c|c|c|c|c|c|c|}
\hline \multirow{4}{*}{$\begin{array}{l}\text { INPUT- } \\
\text { OUTPUT } \\
\text { CLASS. } \\
\text { NUMBER }\end{array}$} & \multirow[t]{4}{*}{ INDUSTRY TITLE } & \multicolumn{6}{|c|}{ REVISIONS } \\
\hline & & \multirow{3}{*}{ ADD } & \multicolumn{3}{|c|}{ ACTION TAKEN } & \multirow{3}{*}{$\begin{array}{l}\text { NUMBER } \\
\text { OF } \\
\text { FIRMS }\end{array}$} & \multirow{3}{*}{$\begin{array}{l}\text { NUMBER } \\
\text { OF } \\
\text { EMPLOYEES }\end{array}$} \\
\hline & & & DROP & OUT OF & NOT & & \\
\hline & & & & AREA & LISTED & & \\
\hline 48.0300 & Wood Working Machinery & $\mathrm{X}$ & & & & 11 & 739 \\
\hline & & & & $\mathrm{x}$ & & 1 & 83 \\
\hline 48.0400 & Paner Industries Machinerv & $\mathbf{y}$ & & & $\mathrm{x}$ & 1 & 29 \\
\hline 40.0400 & Paper mauscries macninery & $\mathrm{x}$ & & & & 3 & 61 \\
\hline 49.0300 & Blowers and Fans & $\mathrm{x}$ & & & & 4 & 531 \\
\hline 49.0500 & Power Transmission Equipment & $\mathrm{x}$ & & & & 1 & 10 \\
\hline 49.0600 & Industrial Furnaces and Ovens & $\mathrm{x}$ & & & & 2 & 40 \\
\hline 49.0700 & General Industrial Machinery & $\mathrm{x}$ & & & & 3 & 79 \\
\hline 51.0300 & Scales and Balances & $\mathrm{x}$ & & & & 1 & 12 \\
\hline 51.0400 & Office Machines n.e.c. & $\mathrm{x}$ & & & & 1 & 1 \\
\hline 52.0100 & Automatic Merchandising Machines & $\mathrm{x}$ & & & & 1 & 1 \\
\hline 50.0001 & $\begin{array}{l}\text { Carburetors, Pistons, Rings, } \\
\text { Valves }\end{array}$ & $\mathrm{X}$ & & & & 1 & 200 \\
\hline 50.0002 & Machinery Except Electrical & $\mathrm{X}$ & & & & 2 & 20 \\
\hline & n.e.c. & & & & $\mathrm{x}$ & 1 & 4 \\
\hline 53.0200 & Transformer:s & $\mathrm{x}$ & & & & 2 & 358 \\
\hline
\end{tabular}


TABLE XIX (CON'T)

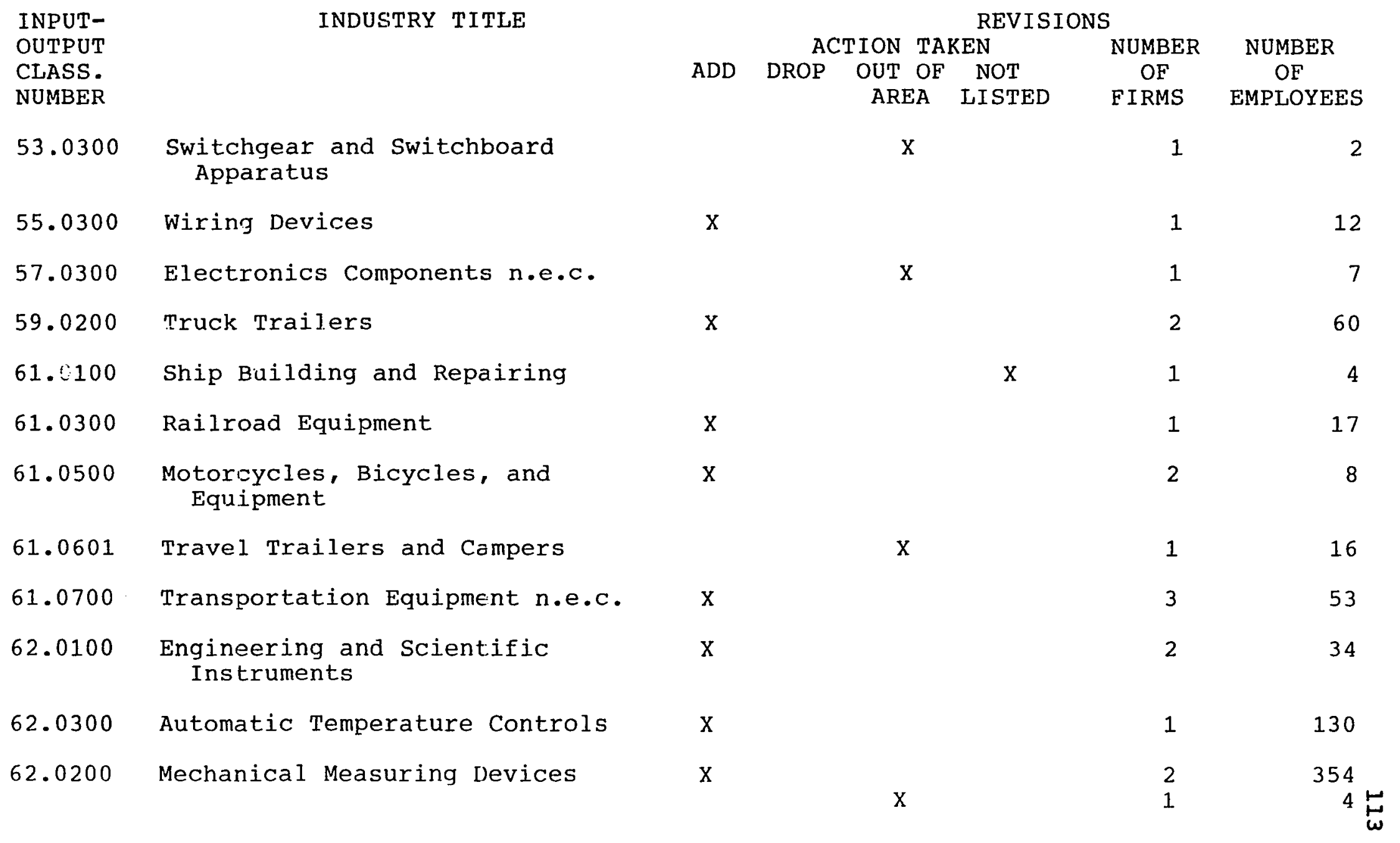


TABLE XIX (CON'T)

\begin{tabular}{|c|c|c|c|c|c|c|c|}
\hline INPUT- & INDUSTRY TITLE & & & & REVISI & & \\
\hline OUTPUT & & & & TION TAF & KEN & NUMBER & NUMBER \\
\hline CLASS . & & ADD & DROP & OUT OF & NOT & OF & OF \\
\hline NUMBER & & & & AREA & LISTED & FIRMS & EMPLOYEES \\
\hline 53.0100 & $\begin{array}{l}\text { Instruments to Measure } \\
\text { Electricity }\end{array}$ & & & & $\mathrm{x}$ & 1 & 1 \\
\hline 62.0600 & Dental Equipment and Supplies & $\mathrm{x}$ & & & & 1 & 5 \\
\hline 63.0200 & Ophthalmic Goods & & & & $\mathrm{x}$ & 1 & 2 \\
\hline 63.0300 & $\begin{array}{l}\text { Photographic Equipment and } \\
\text { Supplies }\end{array}$ & $\mathrm{x}$ & & & & 2 & 41 \\
\hline 64.0200 & Musical Instruments & $\mathrm{x}$ & & & & 2 & 221 \\
\hline 64.0400 & Sporting and Athletic Goods & & & $\mathrm{x}$ & & 1 & 8 \\
\hline 64.0503 & Marking Devices & $\mathrm{x}$ & & & & 5 & 26 \\
\hline 64.0702 & Needles, Pins, and Fasteners & $\mathrm{x}$ & & & & 1 & 8 \\
\hline 64.1000 & Burial Caskets and Vaults & $\mathrm{x}$ & & & & 1 & 15 \\
\hline 64.1200 & Manufacturing Industries n.e.c. & & & & $\mathrm{X}$ & 1 & 2 \\
\hline
\end{tabular}


TABLE XX

IDENTIFICATION OF MANUFACTURING

INDUSTRIES

\begin{tabular}{|c|c|c|c|c|}
\hline \multirow{3}{*}{$\begin{array}{l}\text { INPUT- } \\
\text { OUTPUT } \\
\text { CLASSIF } \\
\text { NUMBER }\end{array}$} & \multirow{3}{*}{$\begin{array}{l}\text { STANDARD } \\
\text { INDUSTRIAL } \\
\text { CODE } \\
\text { NUMBER }\end{array}$} & \multirow[t]{3}{*}{ INDUSTRY TITLE } & \multicolumn{2}{|c|}{$\begin{array}{l}\text { NUMBER } \\
\text { OF }\end{array}$} \\
\hline & & & MS & EMPLOYEES \\
\hline & & & & \\
\hline 14.0101 & 2011 & Meat Packing Plants & 11 & 452 \\
\hline 14.0102 & 2013 & $\begin{array}{l}\text { Sausages and Other } \\
\text { Preparea Meats }\end{array}$ & 8 & 282 \\
\hline 14.0104 & 2017 & $\begin{array}{l}\text { Poultry and Egg } \\
\text { Processing }\end{array}$ & 6 & 230 \\
\hline 14.020 & 2021 & Creamery Butter & 2 & 23 \\
\hline 14.0600 & 2026 & Fluid Milk & 8 & 740 \\
\hline 14.0800 & 2032 & Canned Specialties & 5 & 112 \\
\hline 14.0900 & 2033 & $\begin{array}{l}\text { Canned Fruits and } \\
\text { Vegetables }\end{array}$ & 5 & 97 \\
\hline 14.1000 & 2034 & $\begin{array}{l}\text { Dehydrated Food } \\
\text { Products }\end{array}$ & 2 & 33 \\
\hline 14.1100 & 2035 & $\begin{array}{l}\text { Pickles, Sauces, and } \\
\text { Salad Dressings }\end{array}$ & 5 & 516 \\
\hline 14.1300 & $\begin{array}{l}2037 \\
2038\end{array}$ & $\begin{array}{l}\text { Frozen Fruits and } \\
\text { Vegetables }\end{array}$ & 8 & 715 \\
\hline 14.1401 & 2041 & $\begin{array}{l}\text { Flour and Other Grain } \\
\text { Mill Products }\end{array}$ & 3 & 171 \\
\hline 14.1501 & 2047 & $\begin{array}{l}\text { Dog, Cat, and Other } \\
\text { Pet Food }\end{array}$ & 2 & 246 \\
\hline 14.1502 & 2048 & Prepared Foods, n.e.c. & 8 & 252 \\
\hline 14.1801 & 2051 & $\begin{array}{l}\text { Bread, Cake, and } \\
\text { Related Products }\end{array}$ & 19 & 1,399 \\
\hline 14.1802 & 2052 & Cookies and Crackers & 5 & 1,030 \\
\hline
\end{tabular}


TABLE XX (CON'T)

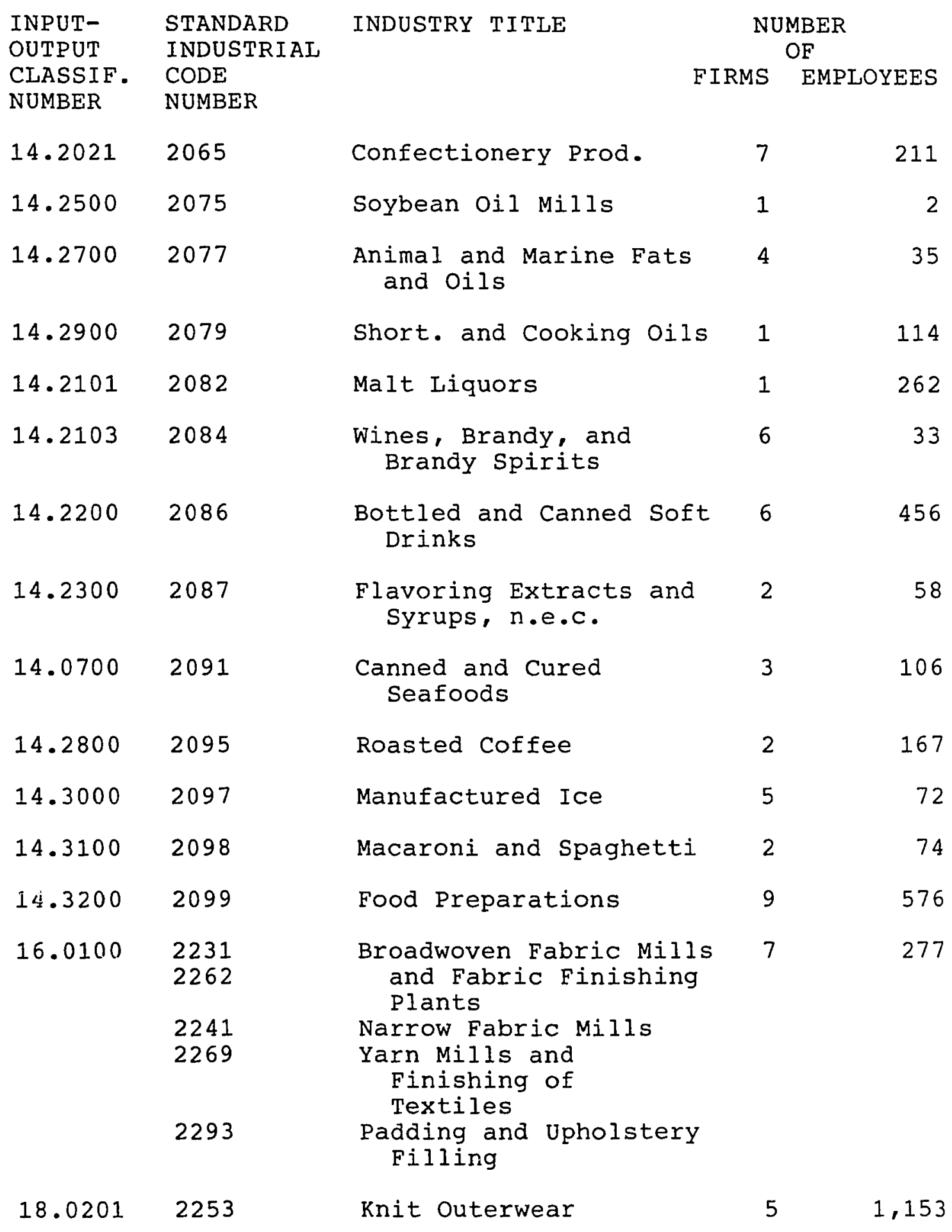


TABLE XX (CON'T)

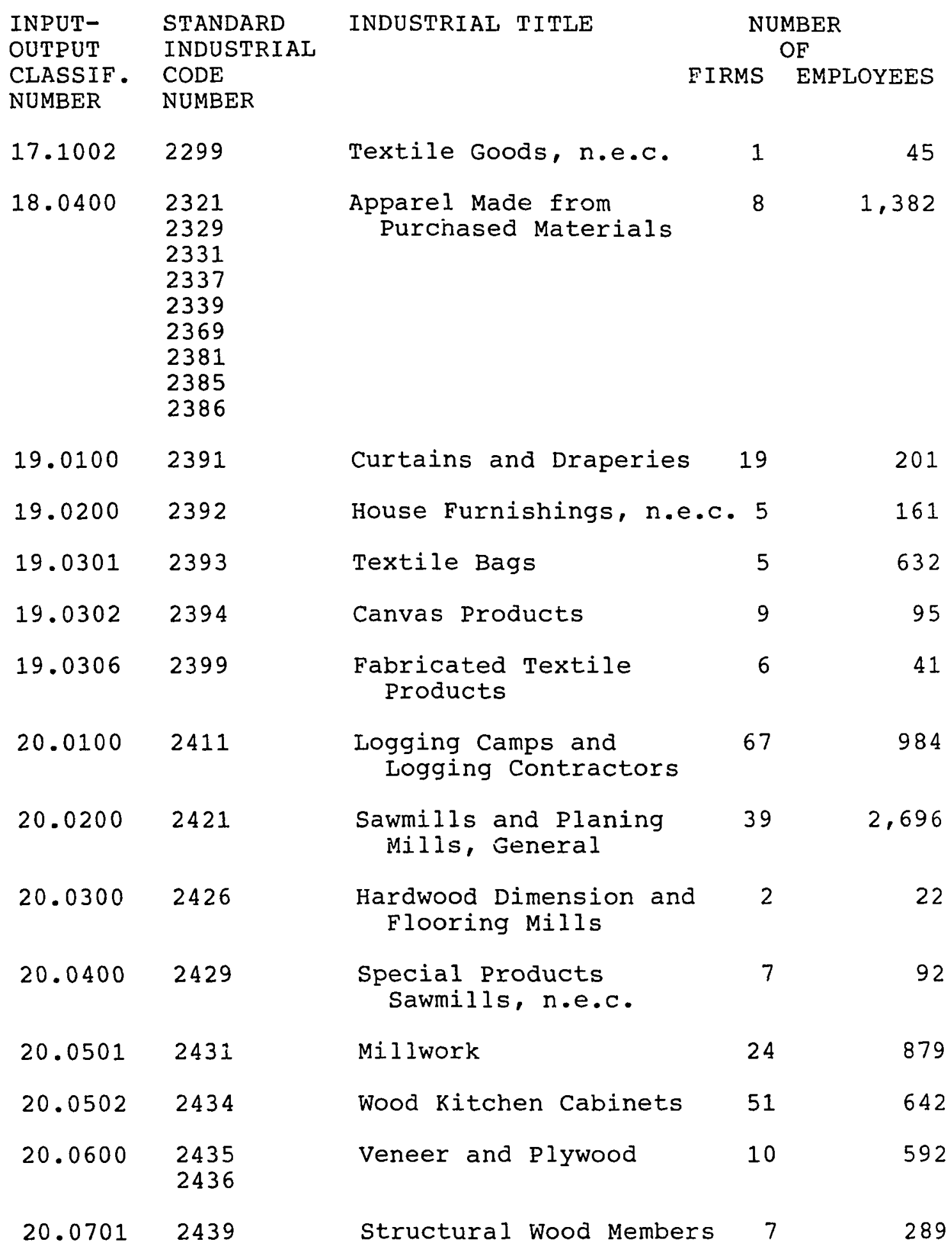


TABLE XX (CON'T)

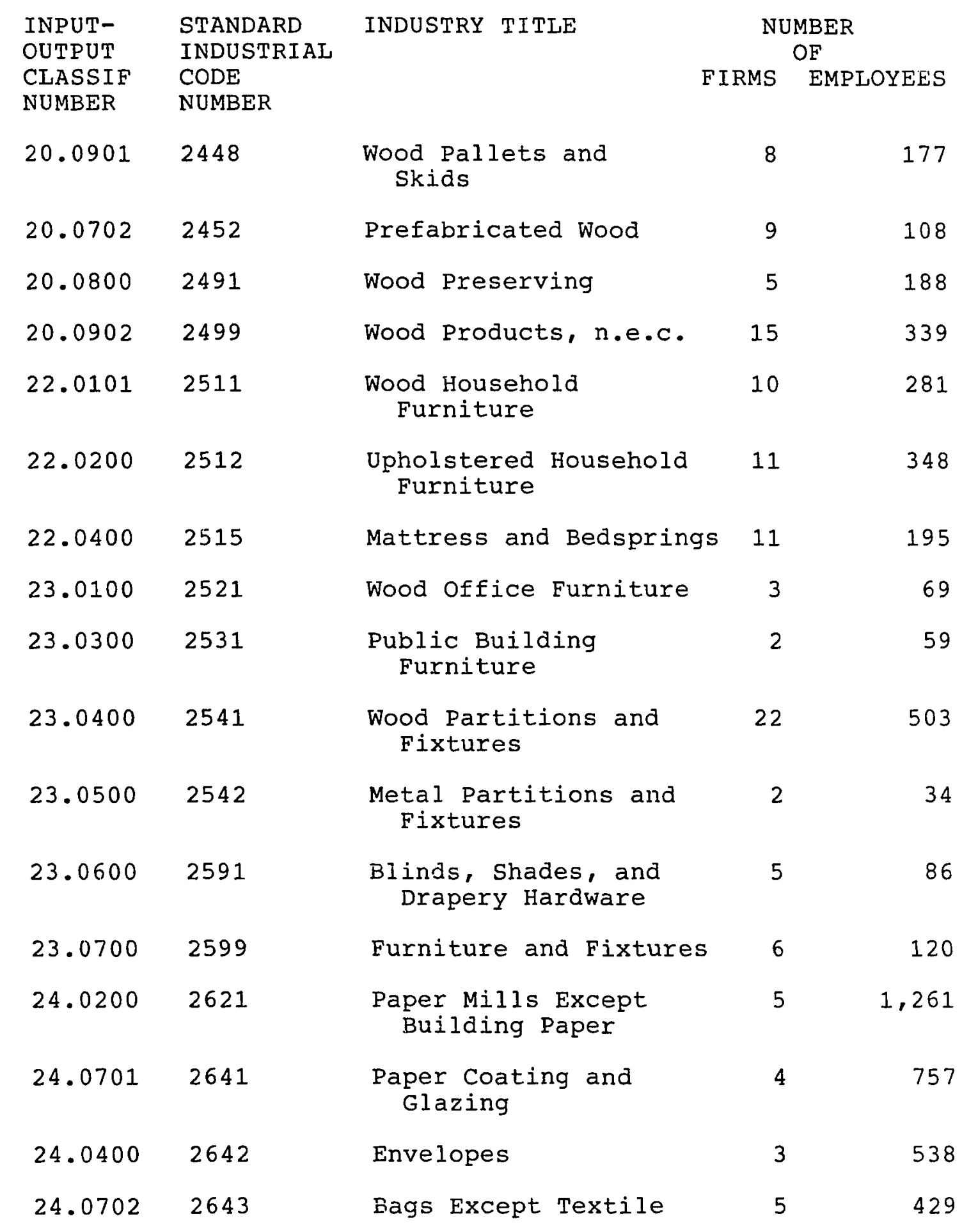


TABLE XX (CON'T)

\begin{tabular}{|c|c|c|c|c|}
\hline $\begin{array}{l}\text { INPUT- } \\
\text { OUTPUT }\end{array}$ & $\begin{array}{l}\text { STANDARD } \\
\text { INDUSTRIAL }\end{array}$ & INDUSTRY TITLE & \multicolumn{2}{|c|}{$\begin{array}{l}\text { NUMBER } \\
\text { OF }\end{array}$} \\
\hline $\begin{array}{l}\text { CLASSIF } \\
\text { NUMBER }\end{array}$ & $\begin{array}{l}\text { CODE } \\
\text { NUMBER }\end{array}$ & & I RMS & EMPLOYEES \\
\hline 24.0704 & 2646 & $\begin{array}{l}\text { Pressed and Molded } \\
\text { Pulp Goods }\end{array}$ & 2 & 62 \\
\hline 24.0706 & 2649 & $\begin{array}{l}\text { Converted Paper } \\
\text { Products n.e.c. }\end{array}$ & 3 & 87 \\
\hline 25.0000 & $\begin{array}{l}2651 \\
2652 \\
2653 \\
2654 \\
2655\end{array}$ & Paperboard Containers & 11 & 716 \\
\hline 26.0100 & 2711 & Newspapers & 32 & 2,115 \\
\hline 26.0200 & 2721 & Periodicals & 20 & 110 \\
\hline 26.0301 & 2731 & Book Publishing & 9 & 51 \\
\hline 26.0400 & 2741 & Miscellaneous Printing & 8 & 53 \\
\hline 26.0501 & $\begin{array}{l}2751 \\
2752\end{array}$ & Commercial Printing & 171 & 1,878 \\
\hline 26.0801 & 2753 & $\begin{array}{l}\text { Engraving and Plate } \\
\text { Printing }\end{array}$ & 5 & 110 \\
\hline 26.0601 & 2761 & Manifold Business Forms & 7 & 258 \\
\hline 26.0602 & 2782 & $\begin{array}{l}\text { Blank Books and Loose } \\
\text { Leaf Binders }\end{array}$ & 7 & 390 \\
\hline 26.0802 & 2789 & $\begin{array}{l}\text { Book Binding and } \\
\text { Related Work }\end{array}$ & 6 & 101 \\
\hline 26.0803 & 2791 & Typesetting & 23 & 141 \\
\hline 26.0804 & 2793 & Photoengraving & 5 & 54 \\
\hline 26.0502 & 2795 & $\begin{array}{l}\text { Lithographic Plate } \\
\text { Making and Services }\end{array}$ & 5 & 53 \\
\hline 27.0100 & 2812 & Industrial Inorganic & 7 & 608 \\
\hline
\end{tabular}




\section{TABLE XX (CON'T)}

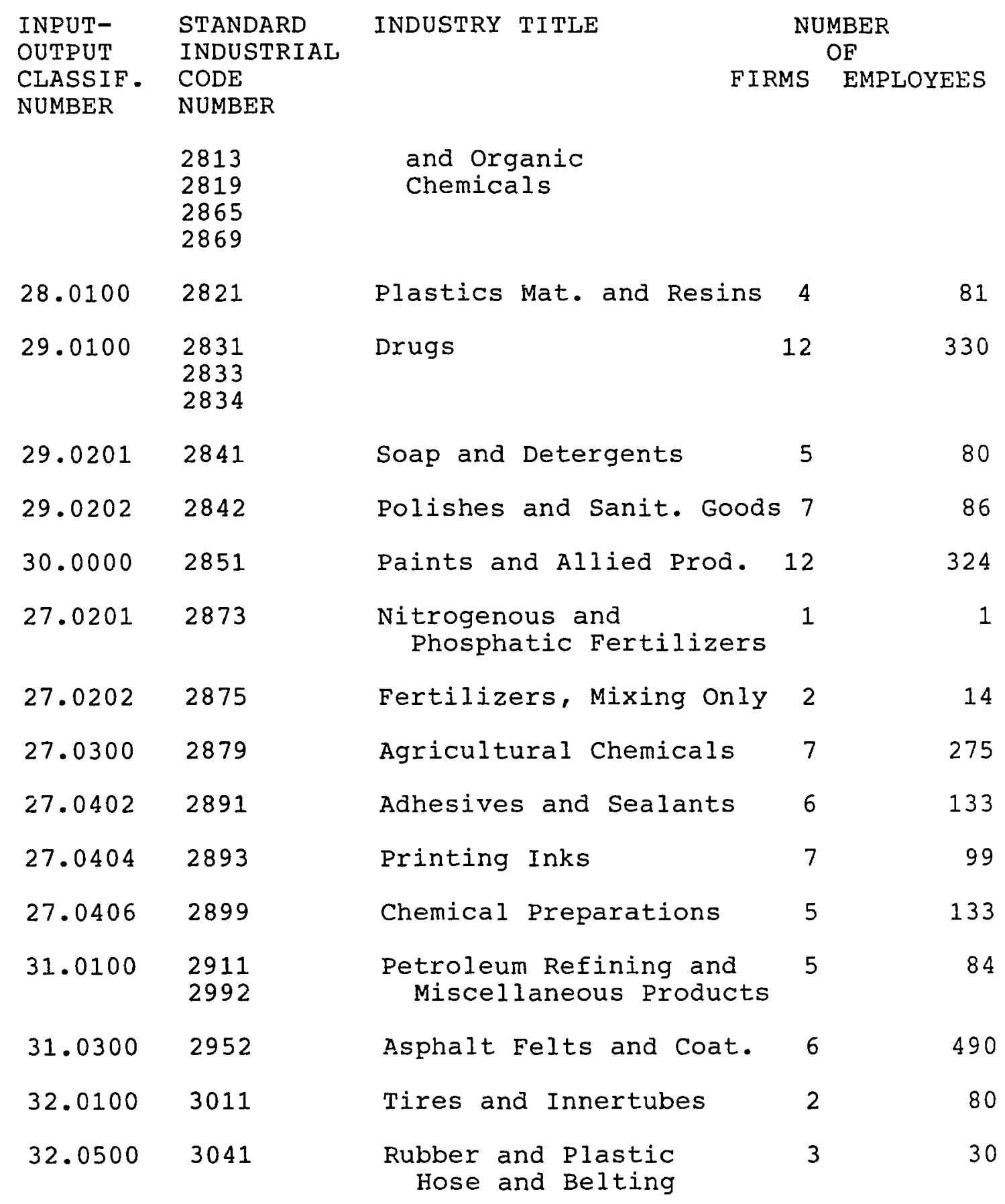


TABLE XX (CON'T)

\begin{tabular}{|c|c|c|c|c|}
\hline $\begin{array}{l}\text { INPUT- } \\
\text { OUTPUT }\end{array}$ & $\begin{array}{l}\text { STANDARD } \\
\text { INDUSTRIAL }\end{array}$ & INDUSTRY TITLE & & $\begin{array}{l}\text { MBER } \\
\text { OF }\end{array}$ \\
\hline CLASSIF . & CODE & & EIRMS & EMPLOYEES \\
\hline 32.0302 & 3069 & Fab. Rubber Prod. & 7 & 409 \\
\hline 32.0400 & 3079 & $\begin{array}{l}\text { Miscellaneous } \\
\text { Plastic Products }\end{array}$ & 55 & 1,386 \\
\hline 33.0001 & 3111 & $\begin{array}{l}\text { Leather Tanning and } \\
\text { Finishing }\end{array}$ & 2 & 50 \\
\hline 34.0201 & $\begin{array}{l}3143 \\
3149\end{array}$ & Shoes Except Rubber & 2 & 55 \\
\hline 34.0301 & 3151 & Leather Gloves, Mitten & 2 & 37 \\
\hline 34.0305 & 3199 & Leather Goods, n.e.c. & 6 & 80 \\
\hline 35.0100 & $\begin{array}{l}3211 \\
3229 \\
3231\end{array}$ & $\begin{array}{l}\text { Glass and Glass } \\
\text { Products Except } \\
\text { Containers }\end{array}$ & 5 & 84 \\
\hline 35.0200 & 3221 & Glass Containers & 2 & 538 \\
\hline 36.0100 & 3241 & Cement, Hydraulic & 2 & 192 \\
\hline 36.0200 & 3251 & $\begin{array}{l}\text { Brick and Structural } \\
\text { Clay Tile }\end{array}$ & 3 & 68 \\
\hline 36.0500 & 3259 & $\begin{aligned} \text { Structural clay } \\
\text { Products n.e.c. }\end{aligned}$ & 2 & 16 \\
\hline 36.0900 & 3269 & Pottery Products & 5 & 126 \\
\hline 36.1000 & 3271 & $\begin{array}{l}\text { Concrete Block and } \\
\text { Brick }\end{array}$ & 2 & 38 \\
\hline 36.1100 & 3272 & Concrete Products, & 21 & 403 \\
\hline 36.1200 & 3273 & Ready-Mixed Concrete & 18 & 576 \\
\hline 36.1300 & 3274 & Lime & 2 & 36 \\
\hline 36.1500 & 3281 & $\begin{array}{l}\text { Cut stone and Stone } \\
\text { Products }\end{array}$ & 4 & 4 \\
\hline
\end{tabular}


TABLE XX (CON'T)

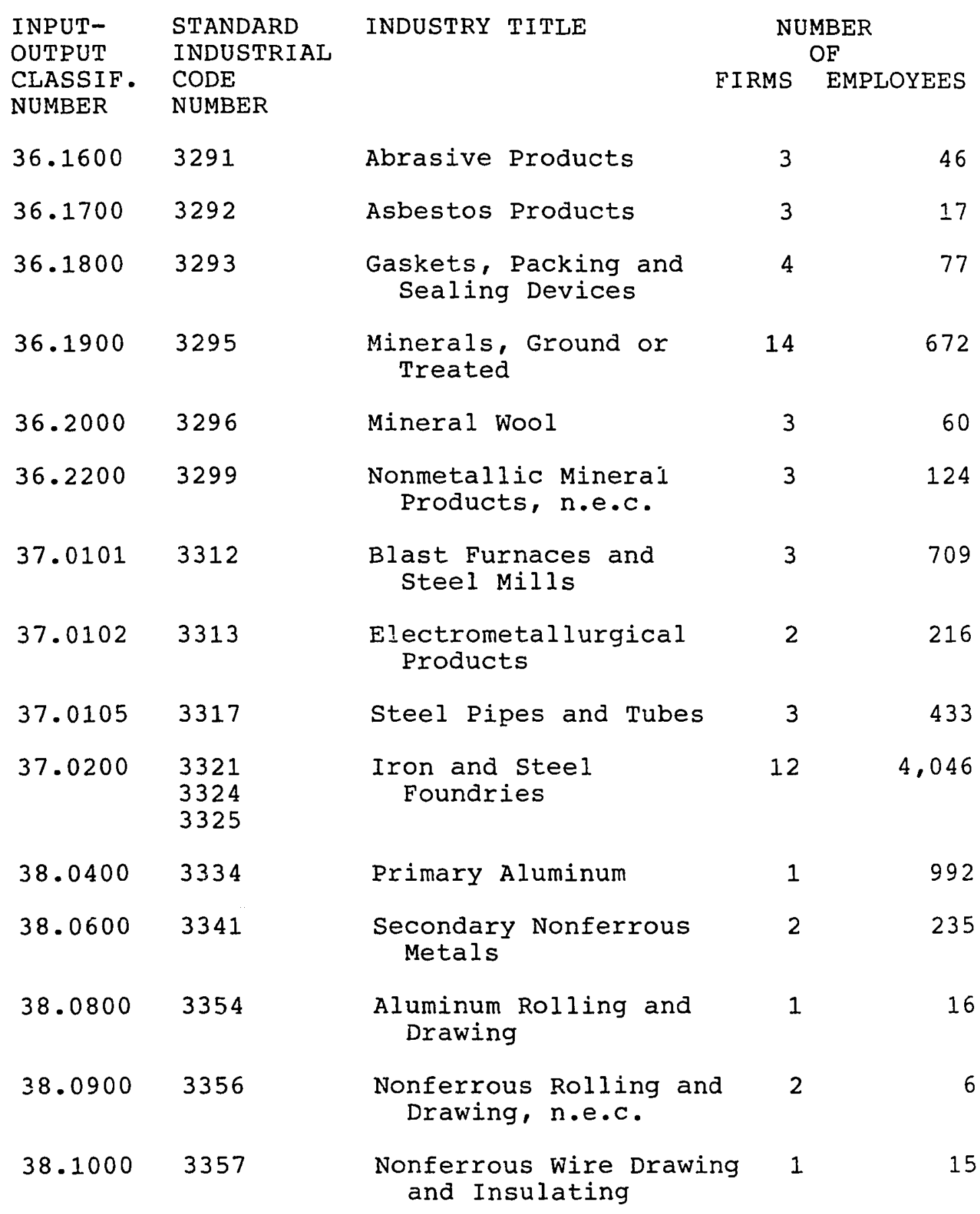


TABLE XX (CON'T)

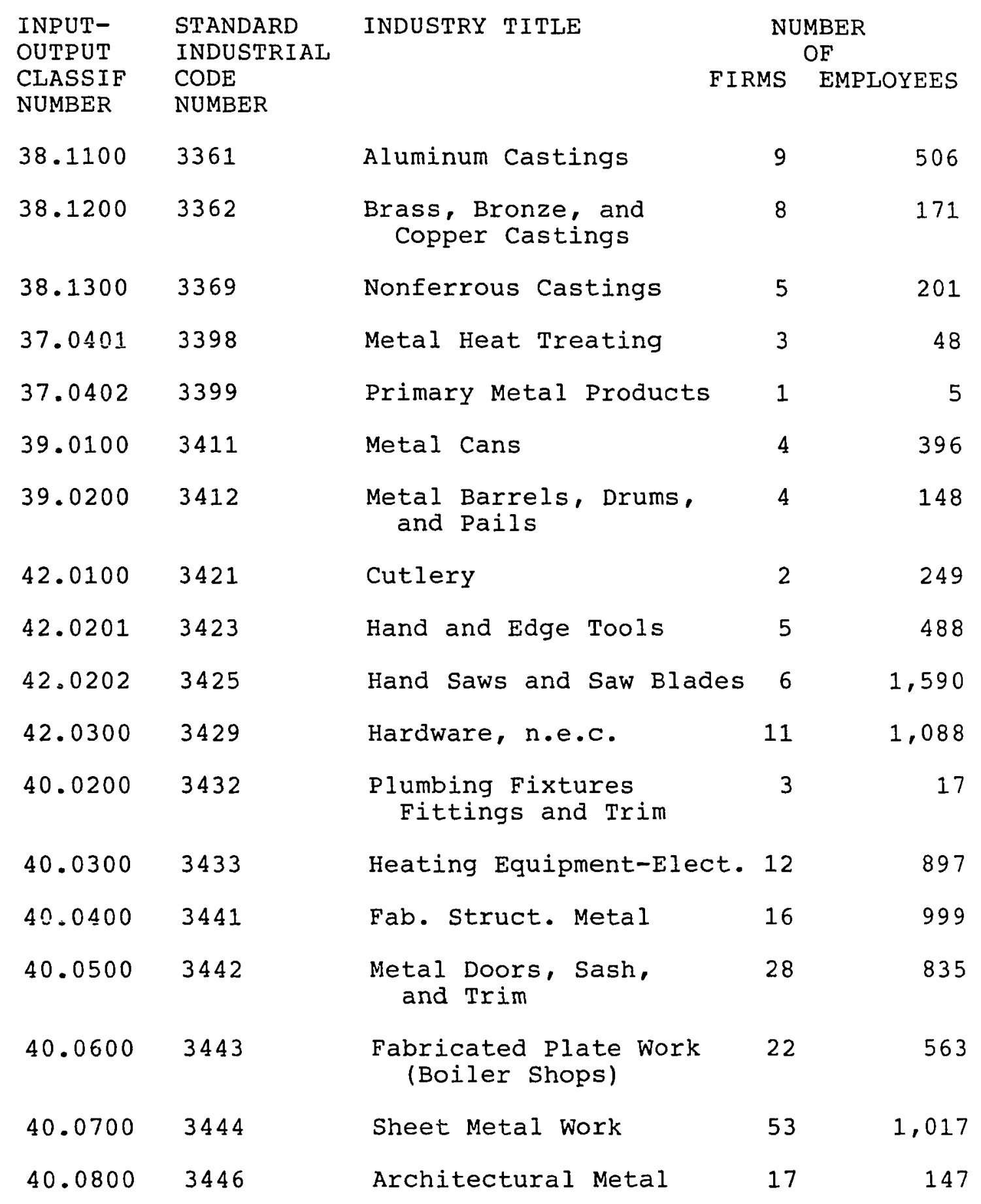


TABLE XX (CON'T)

\begin{tabular}{|c|c|c|c|c|}
\hline $\begin{array}{l}\text { INPUT- } \\
\text { OUTPUT }\end{array}$ & $\begin{array}{l}\text { STANDARD } \\
\text { INDUSTRIAL }\end{array}$ & INDUSTRY TITLE & \multicolumn{2}{|c|}{$\begin{array}{l}\text { NUMBER } \\
\text { OF }\end{array}$} \\
\hline $\begin{array}{l}\text { CLASSIF. } \\
\text { NUMBER }\end{array}$ & $\begin{array}{l}\text { CODE } \\
\text { NUMBER }\end{array}$ & & MS & EMPLOYEES \\
\hline 40.0901 & 3448 & Prefab. Metal Bldgs. & 3 & 59 \\
\hline 41.0100 & $\begin{array}{l}3451 \\
3452\end{array}$ & $\begin{array}{l}\text { Screw Machine Products } \\
\text { and Bolts, Nuts, } \\
\text { Rivets, and Washers }\end{array}$ & 8 & 273 \\
\hline 37.0300 & 3462 & Iron and steel Forgings & 3 & 1.39 \\
\hline 41.0203 & 3469 & Metal Stampings, n.e.c. & 6 & 142 \\
\hline 42.0401 & 3471 & Plating and Polishing & 17 & 604 \\
\hline 42.0402 & 3479 & $\begin{array}{l}\text { Metal Coating and } \\
\text { Allied Services }\end{array}$ & 11 & 273 \\
\hline 13.0600 & 3482 & Small Arms Ammunition & 1 & 5 \\
\hline 13.0500 & 3484 & Small Arms & 4 & 44 \\
\hline 42.0700 & 3493 & $\begin{array}{l}\text { Steel Springs, Except } \\
\text { Wire }\end{array}$ & 6 & 113 \\
\hline 42.0800 & $\begin{array}{l}3494 \\
3498\end{array}$ & $\begin{array}{l}\text { Pipe Valves and Pipe } \\
\text { Fittings }\end{array}$ & $: 4$ & 795 \\
\hline 42.0500 & 3496 & $\begin{array}{l}\text { Miscellaneous Fab } \\
\text { Wire Products }\end{array}$ & 8 & 229 \\
\hline 42.1100 & 3499 & $\begin{array}{l}\text { Fabricated Metal } \\
\text { Products, n.e.c. }\end{array}$ & 5 & 25 \\
\hline 44.0001 & 3523 & $\begin{array}{l}\text { Farm Machinery and } \\
\text { Equipment }\end{array}$ & 11 & 604 \\
\hline 44.0002 & 3524 & Lawn and Garden Equip. & 2 & 17 \\
\hline 45.0100 & 3531 & $\begin{array}{l}\text { Construction Machinery } \\
\text { and Equipment }\end{array}$ & 16 & 32 \\
\hline 45.0200 & 3532 & $\begin{array}{l}\text { Mining Machinery, } \\
\text { Except Oilfield }\end{array}$ & 3 & 6 \\
\hline
\end{tabular}


TABLE I (CON'T)

\begin{tabular}{|c|c|c|c|c|}
\hline $\begin{array}{l}\text { INPUT- } \\
\text { OUTPUT } \\
\text { CLASSIF } \\
\text { NUMBER }\end{array}$ & $\begin{array}{l}\text { STANDARD } \\
\text { INDUSTRIAL } \\
\text { CODE } \\
\text { NUMBER }\end{array}$ & INDUSTRY CODE & & $\begin{array}{l}\text { NUMBER } \\
\quad \text { OF } \\
\text { EMPLOYEES }\end{array}$ \\
\hline 46.0100 & 3534 & $\begin{array}{l}\text { Elevators and Moving } \\
\text { Stairways }\end{array}$ & 2 & 29 \\
\hline 46.0200 & 3535 & $\begin{array}{c}\text { Conveyors and Conveying } \\
\text { Equipment }\end{array}$ & 9 & 314 \\
\hline 46.0300 & 3536 & $\begin{array}{l}\text { Hoists, Cranes, and } \\
\text { Monorails }\end{array}$ & 5 & 218 \\
\hline 46.0400 & 3537 & $\begin{array}{l}\text { Industrial Trucks and } \\
\text { Tractors }\end{array}$ & 15 & 1,907 \\
\hline 47.0100 & 3541 & $\begin{array}{l}\text { Machine Tools, Metal } \\
\text { Cutting Types }\end{array}$ & 4 & 71 \\
\hline 47.0300 & $\begin{array}{l}3544 \\
3545\end{array}$ & $\begin{array}{l}\text { Special Dies and Tools } \\
\text { and Machine Tool } \\
\text { Accessories }\end{array}$ & 19 & 173 \\
\hline 47.0401 & 3546 & Power Driven Hand Tools & 4 & 210 \\
\hline 48.0100 & 3551 & Food Products Machinery & 4 & 32 \\
\hline 48.0300 & 3553 & Woodworking Machinery & 34 & 1,635 \\
\hline 48.0400 & 3554 & $\begin{array}{l}\text { Paper Industries } \\
\text { Machinery }\end{array}$ & 5 & 187 \\
\hline 48.0500 & 3555 & Printing Trades Machinery & 2 & 80 \\
\hline 48.0600 & 3559 & $\begin{array}{l}\text { Special Industry } \\
\text { Machinery, n.e.c. }\end{array}$ & 8 & 342 \\
\hline 49.0100 & 3561 & Pumps and Compressors & 6 & 1,097 \\
\hline 49.0300 & 3564 & Blowers and Fans & 6 & 822 \\
\hline 49.0400 & 3565 & Industrial Patterns & 12 & 134 \\
\hline 49.0500 & $\begin{array}{l}3566 \\
3568\end{array}$ & $\begin{array}{l}\text { Power Transmission } \\
\text { Equipment }\end{array}$ & 3 & 33 \\
\hline
\end{tabular}


TABLE XX (CON'T)

\begin{tabular}{|c|c|c|c|c|}
\hline $\begin{array}{l}\text { INPUT- } \\
\text { OUTPUT }\end{array}$ & $\begin{array}{l}\text { STANDARD } \\
\text { INDUSTRIAL }\end{array}$ & INDUSTRY TITLE & \multicolumn{2}{|c|}{$\begin{array}{l}\text { NUMBER } \\
\text { OF }\end{array}$} \\
\hline $\begin{array}{l}\text { CLASSIF. } \\
\text { NUMBER }\end{array}$ & $\begin{array}{l}\text { CODE } \\
\text { NUMBER }\end{array}$ & & RS & EMPLOYEES \\
\hline 49.0600 & 3567 & $\begin{array}{l}\text { Industrial Furnaces } \\
\text { and Ovens }\end{array}$ & 5 & 136 \\
\hline 49.0700 & 3569 & General Industrial Mach. & 5 & 94 \\
\hline 51.0100 & 3573 & $\begin{array}{l}\text { Electronic Computing } \\
\text { Equipment }\end{array}$ & 5 & 1,904 \\
\hline 51.0300 & 3576 & Scales and Balances & 2 & 14 \\
\hline 51.0400 & 3579 & Office Machines, n.e.c. & 2 & 5 \\
\hline 52.0100 & 3581 & $\begin{array}{l}\text { Automatic Merchandising } \\
\text { Machines }\end{array}$ & 2 & 18 \\
\hline 52.0300 & 3585 & $\begin{array}{l}\text { Refrigeration and } \\
\text { Heating Equipment }\end{array}$ & 5 & 156 \\
\hline 52.0500 & 3589 & $\begin{array}{l}\text { Service Industry } \\
\text { Machines, n.e.c. }\end{array}$ & 7 & 190 \\
\hline 50.0001 & 3592 & $\begin{array}{l}\text { Carburetors, Pistons } \\
\text { Rings, Valves }\end{array}$ & 2 & 201 \\
\hline 50.0002 & 3599 & $\begin{array}{l}\text { Machinery, Except } \\
\text { Electrical, n.e.c. }\end{array}$ & 103 & 1,821 \\
\hline 53.0200 & 3612 & Transformers & 4 & 435 \\
\hline 53.0300 & 3613 & $\begin{array}{l}\text { Switchgear and } \\
\text { Switchboard Apparatus }\end{array}$ & 8 & 412 \\
\hline 53.0500 & 3622 & Industry Controls & 9 & 135 \\
\hline 54.0500 & 3635 & $\begin{array}{l}\text { Household Vacuum } \\
\text { Cleaners }\end{array}$ & 1 & \\
\hline 55.0300 & $\begin{array}{l}3643 \\
3644\end{array}$ & Wiring Devices & 4 & 150 \\
\hline 55.0200 & 3645 & Lighting Fixtures and & 7 & 7 \\
\hline
\end{tabular}


TABLE XX (CON'T)

\begin{tabular}{|c|c|c|c|c|}
\hline \multirow{3}{*}{$\begin{array}{l}\text { INPUT- } \\
\text { OUTPUT } \\
\text { CLASSIE. } \\
\text { NUMBER }\end{array}$} & $\begin{array}{l}\text { STANDARD } \\
\text { INDUSTRIAL }\end{array}$ & \multirow{2}{*}{$\begin{array}{c}\text { INDUSTRY TITLE } \\
\cdot\end{array}$} & \multicolumn{2}{|c|}{$\begin{array}{l}\text { NUMBER } \\
\text { OF }\end{array}$} \\
\hline & $\begin{array}{l}\text { CODE } \\
\text { NUMBER }\end{array}$ & & FIRMS & EMPLOYEES \\
\hline & $\begin{array}{l}3646 \\
3647 \\
3648\end{array}$ & and Equipment & & \\
\hline 56.0100 & 3651 & $\begin{array}{l}\text { Radio and TV Receiving } \\
\text { Sets }\end{array}$ & 9 & 318 \\
\hline 56.0200 & 3652 & $\begin{array}{l}\text { Phonographic Records } \\
\text { and Tape }\end{array}$ & 1 & 6 \\
\hline 56.0300 & 3661 & $\begin{array}{l}\text { Telephone and } \\
\text { Telegraph Apparatus }\end{array}$ & 3 & 465 \\
\hline 56.0400 & 3662 & $\begin{array}{l}\text { Radio and TV } \\
\text { Communication } \\
\text { Equipment }\end{array}$ & 11 & 333 \\
\hline 57.0100 & 3672 & Electron Tubes & 1 & 8 \\
\hline 57.0200 & 3674 & $\begin{array}{l}\text { Semiconductor and } \\
\text { Related Devices }\end{array}$ & 4 & 2,812 \\
\hline 57.0300 & $\begin{array}{l}3677 \\
3679\end{array}$ & $\begin{array}{l}\text { Electronic Components, } \\
\text { n.e.c. }\end{array}$ & 16 & 1,184 \\
\hline 58.0100 & 3691 & Storage Batteries & 6 & 379 \\
\hline 58.0300 & 3693 & $\begin{array}{l}\text { X-ray Apparatus and } \\
\text { Tubes }\end{array}$ & 6 & 203 \\
\hline 58.0400 & 3694 & $\begin{array}{l}\text { Engine Electrical } \\
\text { Equipment }\end{array}$ & 7 & 176 \\
\hline 59.0301 & 3711 & Motor Vehicles & 1 & 3,358 \\
\hline 59.0100 & 3713 & Truck and Bus Bodies & 7 & 170 \\
\hline 59.0302 & 3714 & $\begin{array}{l}\text { Motor Vehicle Parts } \\
\text { and Accessories }\end{array}$ & 33 & 972 \\
\hline 59.0200 & 3715 & Truck Trailers & 16 & 619 \\
\hline
\end{tabular}


TABLE XX (CON'T)

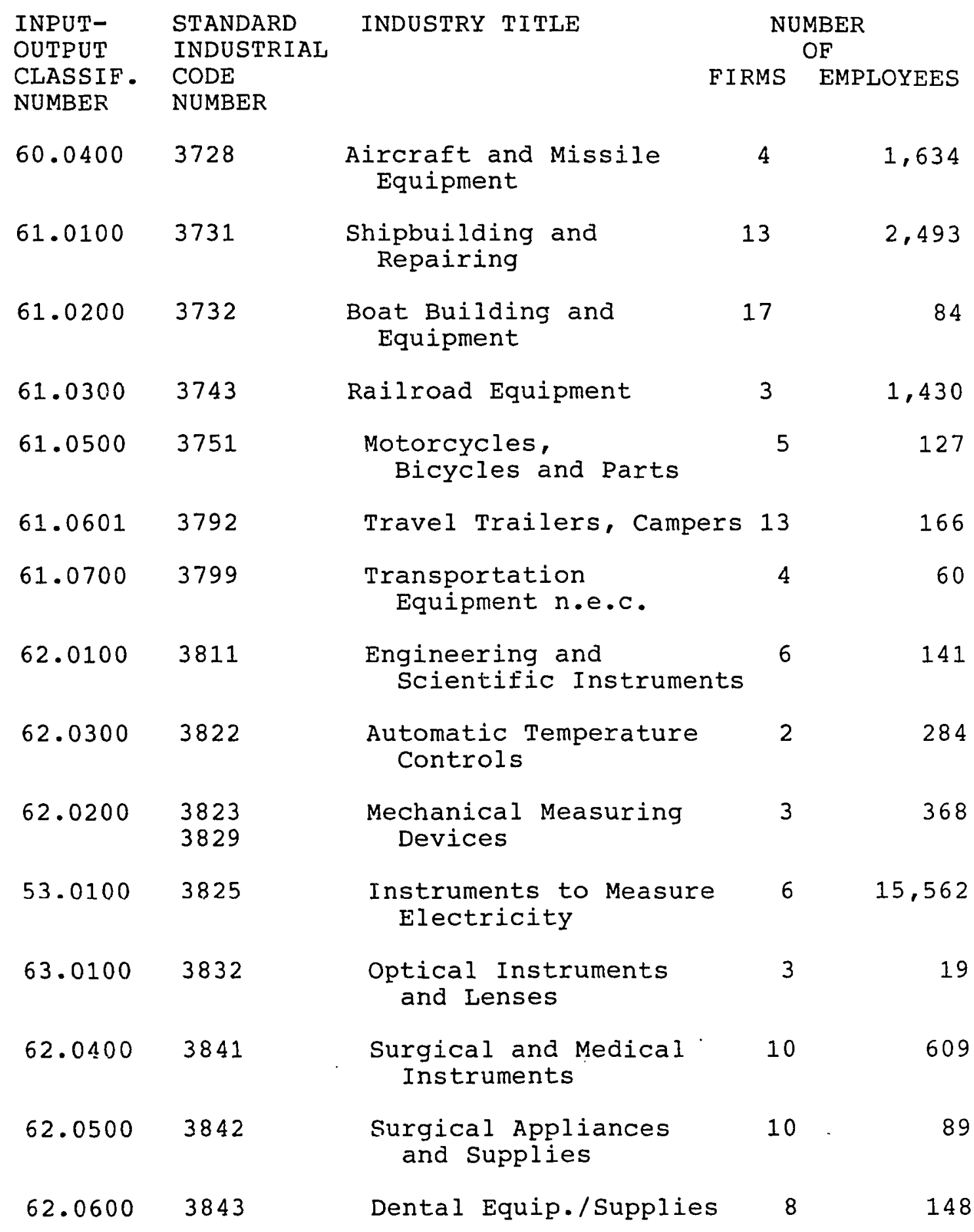




\section{TABLE XX (CON'T)}

\begin{tabular}{|c|c|c|c|c|}
\hline $\begin{array}{l}\text { INPUT- } \\
\text { OUTPUT }\end{array}$ & $\begin{array}{l}\text { STANDARD } \\
\text { INDUSTRIAL }\end{array}$ & INDUSTRY TITLE & \multicolumn{2}{|c|}{$\begin{array}{l}\text { NUMBER } \\
\text { OE }\end{array}$} \\
\hline $\begin{array}{l}\text { CLASSIF } \\
\text { NUMBER }\end{array}$ & $\begin{array}{l}\text { CODE } \\
\text { NUMBER }\end{array}$ & FIR & MS & EMPLOYEES \\
\hline 63.0200 & 3851 & Ophthalmic Goods & 8 & 174 \\
\hline 63.0300 & 3861 & $\begin{array}{l}\text { Photographic Equipment } \\
\text { and Supplies }\end{array}$ & 6 & 900 \\
\hline 62.0700 & 3873 & $\begin{array}{l}\text { Watches, Clocks, and } \\
\text { Parts }\end{array}$ & 2 & 10 \\
\hline 64.0101 & 3911 & Jewelry and Prec. Metals & 10 & 47 \\
\hline 64.0104 & 3914 & $\begin{array}{l}\text { Silverware and } \\
\text { Plated Wear }\end{array}$ & 3 & 28 \\
\hline 64.0200 & 3931 & Musical Instruments & 4 & 228 \\
\hline 64.0302 & 3942 & Dolls & 1 & 5 \\
\hline 64.0400 & 3949 & $\begin{array}{l}\text { Sporting and Athletic } \\
\text { Goods }\end{array}$ & 13 & 339 \\
\hline 64.0503 & 3953 & Marking Devices & 8 & 81 \\
\hline 64.0504 & 3955 & $\begin{array}{l}\text { Carbon Paper and Inked } \\
\text { Ribbons }\end{array}$ & 1 & 26 \\
\hline 64.0105 & 3961 & Costume Jewelry & 2 & 4 \\
\hline 64.0702 & 3964 & $\begin{array}{l}\text { Needles, Pins, and } \\
\text { Fasteners }\end{array}$ & 3 & 23 \\
\hline 64.0800 & 3991 & Brooms and Brushes & 3 & 234 \\
\hline 64.1100 & 3993 & $\begin{array}{l}\text { Signs and Advertising } \\
\text { Displays }\end{array}$ & 16 & 180 \\
\hline 64.1000 & 3995 & Burial Caskets/Vaults & 3 & 21 \\
\hline 64.1200 & 3999 & Manufacturing Ind. nec & 8 & 134 \\
\hline TOT & & & 111 & 108,295 \\
\hline
\end{tabular}

